\title{
Article \\ Non-Proportionality Indices and Error Constraint in Modal Analysis of Viscously Damped Linear Structures
}

\author{
Jorge Conde *(D) and Alejandro Bernabeu
}

check for updates

Citation: Conde, J.; Bernabeu, A. Non-Proportionality Indices and Error Constraint in Modal Analysis of Viscously Damped Linear Structures. Appl. Sci. 2021, 11, 6064. https:// doi.org/10.3390/app11136064

Academic Editor: Antonella D'Alessandro

Received: 17 May 2021

Accepted: 25 June 2021

Published: 29 June 2021

Publisher's Note: MDPI stays neutral with regard to jurisdictional claims in published maps and institutional affiliations.

Copyright: (C) 2021 by the authors. Licensee MDPI, Basel, Switzerland. This article is an open access article distributed under the terms and conditions of the Creative Commons Attribution (CC BY) license (https:// creativecommons.org/licenses/by/ $4.0 /)$.
Departamento de Estructuras y Física de la Edificación, Escuela Técnica Superior de Arquitectura, Universidad Politécnica de Madrid, Av. Juan de Herrera, 4, 28040 Madrid, Spain; alejandro.bernabeu@upm.es

* Correspondence: jorge.conde@upm.es; Tel.: +34-680-128-040

Featured Application: Control of certain non-proportionality indices can be used to limit response errors when classical modal analysis is applied to non-proportionally damped structures.

\begin{abstract}
Use of modal procedures in systems with non-proportional damping (such as structures with added viscous damping systems) results in response errors, shown in this study to depend on dissimilar and often conflicting conditions for different variables and stories; thus, it is not possible to propose simple rules based on structural or damping properties to limit the error in a global way. However, four existing indices (originally proposed to measure damping non-proportionality) present a positive correlation with the extreme errors in modal procedures for all variables and stories. Thus, limiting the index value is a sufficient condition to keep the error in all variables within a given threshold. For practical application, limit values for these indices are tabulated as a function of error and can be used as an acceptance criterion for the validity of modal procedures.
\end{abstract}

Keywords: viscous dampers; modal analysis; response spectrum analysis; non-proportionality indices; proportional damping

\section{Introduction}

In the last decades there has been increasing interest and research on damping devices. When properly added to a structure, these devices improve considerably its dynamic performance [1-3]. Among the different types of dampers, pure viscous dampers present the unique feature that their behavior is solely dependent on velocity, rather than displacement: under forced harmonic excitation their damping forces are out of phase with the elastic restoring forces, improving the energy dissipation capacity of the structure without increasing its stiffness [4,5]. If the damping matrix of the system fulfils certain conditions [6], the natural vibration modes of the damped system are real-valued and identical to those of the associated undamped structure. This type of system is referred to as classically (or proportionally) damped, and its solution by modal superposition is known as 'classical modal method'. To estimate the maximum response of a classically damped structure subjected to earthquake loading, the classical modal method is used in conjunction with response spectra, and this is the basis of the well-known Response Spectrum Analysis (RSA), introduced in FEMA-273 [7] and now included [8] or considered for inclusion [9] in several seismic codes around the world for analysis of structures with added viscous damping systems.

However, placement of viscous dampers generally results in a non-proportional damping matrix, i.e., one that does not result in real-valued natural damped vibration modes. The modal method can also be applied to this type of systems in a generalized form due to Foss [10]. This method, referred to as 'generalized modal method', has found limited application in structural engineering practice, possibly because (i) it is more prolix than 
classical modal analysis; (ii) when used with the response spectrum has to rely on approximations of questionable accuracy; and (iii) the physical meaning of the elements involved in the solution is not as well identified as in the classical modal method [11]. To avoid the complexity involved in the generalized modal method, seismic codes impose conditions on the design of structures with viscous dampers, so that effects of non-proportional damping can be ignored, and the classical modal method can be applied. For example, placement of dampers following a complete vertical distribution throughout the building is assumed to reduce non-proportionality effects [12], whereupon the seismic response can be reasonably estimated with the undamped vibration modes, applying the classical modal method $[4,13]$. For systems with non-proportional damping, this involves ignoring the off-diagonal coefficients of the exact modal damping matrix. The errors resulting from this approximation have been pointed out in previous studies [14], yet there are, to these authors' knowledge, no systematic investigations that quantify these errors; in particular, their relationship with properties of the system, degree of non-proportionality of the damping matrix, or amount of damping. The primary objective of the current study is to quantify this error for linear structures with viscous dampers having different number of stories, fundamental periods, amounts of damping, distributions of dampers and distributions of lateral stiffness. A second objective is to correlate the error with the level of non-proportionality of the damping matrix measured by different indices proposed in the literature. Finally, recommendations are made regarding the value of existing indices that measure the degree of non-proportionality of the damping matrix, so that errors resulting from application of classical modal analysis to non-proportionally damped systems are kept within acceptable bounds. This research is of interest because classical modal methods are systematically used in practice, for example, the Response Spectrum Analysis in ASCE/SEI 7-16 [8], or other simplified procedures relying in representation of the multi-degree-of-freedom (MDOF) structure by equivalent single-degree-of-freedom (SDOF) oscillators such as performance spectra $[15,16]$.

\section{Materials and Methods}

\subsection{Proportional and Non-Proportional Damping}

The dynamic equilibrium equation of a linear lumped system with $N$ degrees of freedom under a single component of base earthquake excitation is [17]:

$$
\mathbf{m} \ddot{\mathbf{u}}(t)+\mathbf{c} \dot{\mathbf{u}}(t)+\mathbf{k u}(t)=-\mathbf{m} \mathbf{\imath} a_{g}(t),
$$

where $\mathbf{u}$ is the displacement vector, $\mathbf{m}$ is the mass matrix, $\mathbf{c}$ is the damping matrix, $\mathbf{k}$ is the stiffness matrix, $\mathrm{t}$ is the influence vector representing displacements in the lumped story masses due to a unit base displacement, and $a_{g}(t)$ is the ground acceleration. Equation (1) can be transformed using the undamped system's modal matrix $\boldsymbol{\Phi}$ (where each column $\boldsymbol{\varphi}_{j}=\left[\varphi_{1 j}, \ldots \varphi_{N j}\right]^{\mathrm{T}}$, corresponds to the $j^{\text {th }}$ vibration mode); applying the change of coordinates $\mathbf{u}(t)=\boldsymbol{\Phi} \mathbf{y}(t)$ (where $\mathbf{y}(t)$ is a $N \times 1$ vector of time-dependent modal coordinates), pre-multiplying by $\boldsymbol{\Phi}^{\mathrm{T}}$ and using the orthogonality properties of natural modes, the expression reads:

$$
\mathbf{M} \ddot{y}(t)+\mathbf{C} \dot{y}(t)+\mathbf{K} y(t)=-\boldsymbol{\Phi}^{\mathrm{T}} \mathbf{m} \mathbf{\imath} a_{g}(t),
$$

where the diagonal matrices $\mathbf{M}=\boldsymbol{\Phi}^{\mathrm{T}} \mathbf{m} \boldsymbol{\Phi}$ and $\mathbf{K}=\boldsymbol{\Phi}^{\mathrm{T}} \mathbf{k} \boldsymbol{\Phi}$ are the modal mass and modal stiffness matrix, respectively, and $\mathbf{C}=\boldsymbol{\Phi}^{\mathrm{T}} \mathbf{c} \boldsymbol{\Phi}$ is the modal damping matrix. A special case of this transformation occurs when the modal matrix is previously normalized to the system's mass matrix, i.e., $\hat{\boldsymbol{\Phi}}^{\mathrm{T}} \mathbf{m} \hat{\boldsymbol{\Phi}}=\mathbf{I}$ (where $\mathbf{I}$ is the $N \times N$ identity matrix and $\hat{\phi}_{j}=\phi_{j} M_{j}^{-0.5}$ ), whereupon:

$$
\ddot{y}(t)+\hat{\mathbf{C}} \dot{y}(t)+\mathbf{\Omega}^{2} y(t)=-\hat{\boldsymbol{\Phi}}^{\mathrm{T}} \mathbf{m} \mathbf{\imath} a_{g}(t) ;
$$


In this expression $\Omega^{2}$ is the diagonal spectral matrix, with $j^{\text {th }}$ term $\omega_{j}^{2}$, where $\omega_{j}$ is the $j^{\text {th }}$ mode undamped circular frequency, and $\hat{\mathbf{C}}$ is referred to as normalized modal damping matrix. If $\hat{\mathbf{C}}$ is a diagonal matrix, Equation (3) results in $N$ uncoupled modal equations:

$$
\ddot{y}_{j}(t)+\hat{C}_{j} \dot{y}_{j}(t)+\omega_{j}^{2} y_{j}(t)=-\hat{\Gamma}_{j} a_{g}
$$

where $\hat{C}_{j}$ is the $j^{\text {th }}$ diagonal term of $\hat{\mathbf{C}}$, and $\hat{\Gamma}_{j}\left(=\hat{\boldsymbol{\phi}}_{j}^{\mathrm{T}} \mathbf{m} \mathbf{\imath}\right)$ is the mass-normalized modal participation factor corresponding to mode $j$. Each mode is thus represented by an independent SDOF system with modal damping ratio $\xi_{j}=\hat{C}_{j} /\left(2 \omega_{j}\right)=C_{j} /\left(2 M_{j} \omega_{j}\right)$. As shown by Caughey and O'Kelly [6], $\hat{\mathbf{C}}$ will be diagonal if, and only if:

$$
\mathbf{c m}^{-1} \mathbf{k}=\mathbf{k m}^{-1} \mathbf{c} .
$$

This is known as the commutability condition and can be met by a Caughey damping matrix $\mathbf{c}$ which is a combination of $\mathbf{m}$ and $\mathbf{k}$ [18]. Mass-proportional damping, stiffnessproportional damping and Rayleigh damping are all special cases of Caughey damping. If Equation (5) is fulfilled, $\mathbf{c}$ is referred to as proportional damping matrix, $\hat{\mathbf{C}}$ is diagonal, and each modal equation given by (4) can be solved independently using time-history modal analysis (THMA) to find the complete modal response for each mode. Addition of modal responses at each time step produces the exact total system response. This is the classical modal method. Conversely, if $\mathbf{c}$ is non-proportional, $\hat{\mathbf{C}}$ is not diagonal and from Equation (3):

$$
\ddot{y}_{j}+\sum_{n=1}^{N} \hat{C}_{j n} \dot{y}_{n}+\omega_{j}^{2} y_{j}=-\hat{\Gamma}_{j} a_{g}(t)
$$

which is a coupled system of second-order differential equations that cannot be solved by the classical modal method, unless the off-diagonal terms in $\hat{\mathbf{C}}(j \neq n)$ are neglected, whereupon an approximate solution can be independently found for each mode. The error in this procedure, produced by neglecting off-diagonal terms in the modal damping matrix, is the main object of this study, and is referred to as 'error 1 '.

A second type of error is related to the way the modal response is found. If only the peak response (maximum of the unsigned complete time-history response) is calculated for each mode $j$, the method is referred to as response spectrum analysis (RSA). Generally peak values obtained for different modes are not simultaneous in time and, to find the total response, peak modal values are combined through combination rules. Two rules are generally used, namely the square-root-of-sum-of-squares (RSA-SRSS) [19] and the complete quadratic combination (RSA-CQC) $[20,21]$. These errors are also treated in this study. Improved combination rules for the specific case of non-proportional damping have been derived [22,23] but are not common engineering practice.

Two other types of error appear in modal analysis: The third type, modal truncation error, appears when only a limited number of modes $J<N$ is considered in analysis. Truncation measures developed specifically for non-classically damped systems, exist in the literature [24]. The fourth type occurs when peak modal responses for damped modes are obtained using 5\%-damped elastic spectral ordinates modified via damping correction factors. A summary of existing proposals for these factors can be found in [25]. In this study, however, all modes are considered, and the spectral values are accurately obtained from damped spectra, therefore these two types of error are not included, and the discussion focuses on errors produced by (i) neglecting off-diagonal terms in the modal damping matrix and (ii) use of combination rules.

\subsection{Indices to Measure Damping Non-Proportionality}

The free-vibration problem of an undamped system is described by Equation (1) with $\mathbf{c}=\mathbf{0}$ and $a_{g}(t)=0$ resulting in an eigenproblem with real-valued solutions $\phi_{j}$ and $\omega_{j}^{2}$. For a damped system, the free-vibration problem is solved transforming the general equation of motion into $2 \mathrm{~N}$ state-space; if the damping matrix $\mathbf{c}$ is non-proportional, the corresponding 
eigenproblem produces complex-valued modes $\psi_{j}$ and frequencies $\lambda_{j}$ [26] (as customary, a different notation, is used to differentiate undamped real-valued vibration properties $\Phi, \Omega$, from damped complex-valued vibration properties $\boldsymbol{\Psi}, \boldsymbol{\Lambda}$; where $\boldsymbol{\Lambda}$ is the complex-valued spectral matrix equivalent to $\Omega$ in damped systems, see Appendix A). The complex nature of modes indicates out-of-phase free vibration of the different degrees-of-freedom [27]. The complex modes can be graphically represented in the complex plane, each mode consisting of $N$ vectors or points that, properly ordered, form a $N$-sided modal polygon. At the same time, as shown in the previous section, the corresponding modal damping matrix $\hat{\mathrm{C}}$ is fully populated, rather than diagonal, and can be expressed as $\hat{\mathbf{C}}=\hat{\mathbf{C}}_{d}+\hat{\mathbf{C}}_{o}$, where $\hat{\mathbf{C}}_{d}$ is diagonal and $\hat{\mathbf{C}}_{o}$ is populated with $\hat{\mathbf{C}}_{o, j j}=0$. The differences between Equations (5) and (6) are due to the relative weight of $\hat{\mathbf{C}}_{o}$ with respect to $\hat{\mathbf{C}}_{d}$.

Several authors have proposed indices to measure the degree of non-proportionality of damped systems. Using these two features (phase properties of the complex modes or modal polygons and relative importance of off-diagonal components of $\hat{\mathbf{C}}$ ). Thus, these non-proportionality indices (hereinafter referred to as NPIs) can be broadly divided in two families: those obtained from modal damping matrix components and those derived from properties of damped vibration modes. The first set is probably more adequate for design, as complex modes are not a usual output of conventional analysis programs, whereas the modal damping matrix of systems with added damping can be easily assembled from first principles. In all cases, a zero value of the index is indicative of proportional damping. Indices upper-bounded to unity are referred to as normal, and are of greater practical value, as they allow direct comparison between different systems. Some of the indices are mode-dependent; for this case, a practical global index can be based in all modes, a single mode, or a subset of the most relevant modes that can be selected by some specific criterion. In this study, the average across modes is used. An interesting question is the type of correlation (if any) between index values and the level of non-proportionality. This topic is addressed by the study described in the next section. The indices considered in this study (ordered first by their nature and then chronologically) are:

Indices based on components or properties of the modal damping matrix:

- $\quad I_{W S 1}, I_{W S 2}$ by Warburton and Soni (1977) [28].

- $\quad I_{P S 5}, I_{P S 6}$ by Prater and Singh (1986) [29].

- $\quad I_{C V 1}$ by Claret and Venancio-Filho (1991) [30].

- $\quad I_{T L L 1}$ by Tong, Liang and Lee (1994) [31].

- $\quad I_{B 1}$ by Bhaskar (1995) [32].

Indices based on properties of the complex modes or modal polygons:

- $\quad I_{P S 1}, I_{P S 2}, I_{P S 3}, I_{P S 4}$ by Prater and Singh (1986) [29].

- $\quad I_{B 2}, I_{B 3}$ by Bhaskar (1999) [33].

- $\quad I_{L K Z 1}, I_{L K Z 2}, I_{L K Z 3}, I_{L K Z 4}, I_{L K Z 5}, I_{L K Z 6}$ by Liu, Kujath and Zheng (2000; 2001) [34,35].

- $\quad I_{P F 1}$ by Prells and Friswell (2000) [36].

- $I_{A 1}$ by Adhikari (2004) [37].

Other indices:

- $\quad$ Nair and Singh (1986), $I_{N S 1}$ [38].

These indices are succinctly described in Appendices A and A.1-A.9. The investigation undertaken regarding these indices concerns the type of correlation (if any) between their values for specific systems and the two types of error discussed in the previous section (suppression of off-diagonal terms in the modal damping matrix; use of combination rules). This topic is addressed by the study described in the next section. Emphasis is made on indices with possibilities to be applied in a practical engineering context, laying aside those that require, for instance, full evaluation of the system's response. As described later, four indices $\left(I_{P S 5}, I_{L K Z 4}, I_{T L L 1}\right.$ and $\left.I_{A 1}\right)$ have been found to be significantly correlated to the error. 


\subsection{Numerical Study}

In this numerical study, non-proportionally damped MDOF structures are subjected to earthquake excitation. Their time-history response is found with either the 'exact' (populated) or the 'approximate' (diagonal) modal damping matrices; in addition, response spectrum analyses are also carried out, using both SRSS and CQC combination rules, with exact spectral responses (so there is no error due to damping correction factors) and including all modes (so there is no error due to modal truncation). A detailed description of the study follows:

\subsubsection{Systems Analyzed}

The planar elastic MDOF structures included in the study can be described by the generic frame shown in Figure 1a, defined by a vertical distribution of column stiffness $k_{c, e q, n}$, beam stiffness $k_{b, e q, n}$, and story mass $m_{n}=0.25 \mathrm{kN} \cdot \mathrm{s}^{2} / \mathrm{mm}$ equal at all stories. For any element $x$ (beam or column), $k_{e q, x}=E I_{e q, x} / L_{x}$, where $E$ is the Young modulus ( $210 \mathrm{GPa}$ for all elements), $I_{e q, x}$ is the element's second moment of inertia associated to in-plane displacement, and $L_{x}$ is the element centerline length. Subscript 'eq' is used because the one-bay frame in Figure 1a can be seen as a surrogate for a multi-bay frame, as described in Appendix B. The axial and shear flexibility of the members is neglected, as is the outof-plane behavior, and it is assumed that $h_{h}=h, L=2 h$, and the ratio between beam and column stiffness $\lambda$ is constant for the whole frame:

$$
\lambda_{n}=E I_{b, e q, n} / E I_{c, e q, n}=2 k_{b, e q, n} / k_{c, e q, n}=\lambda .
$$

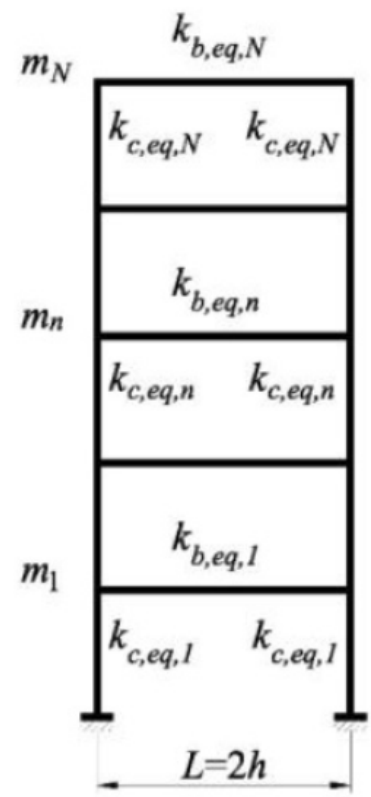

(a)

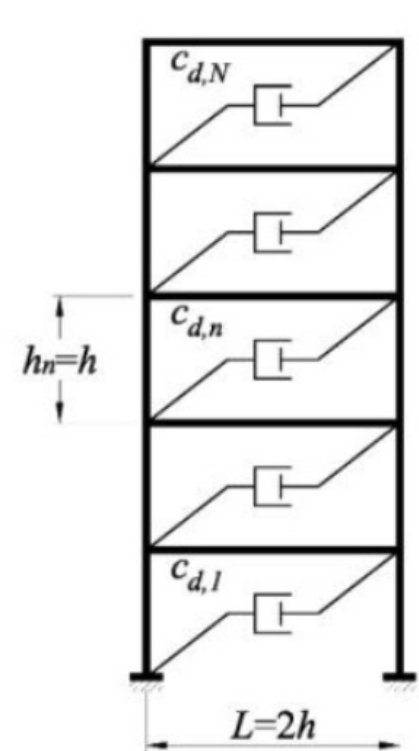

(b)

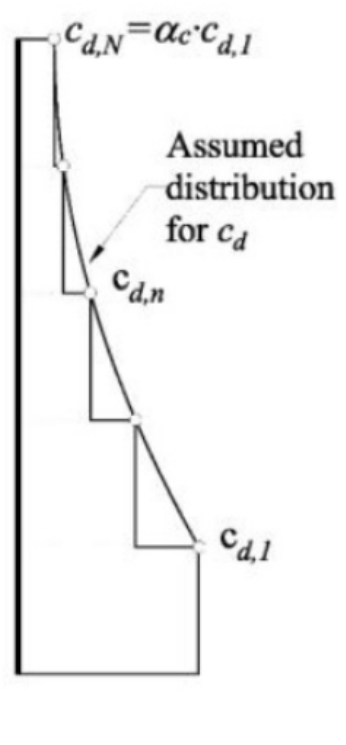

(c)

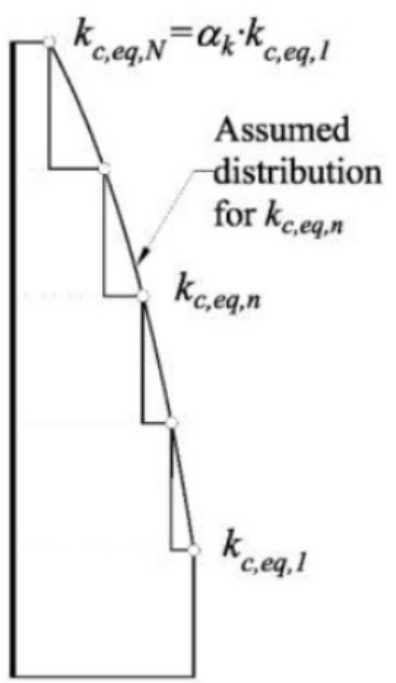

(d)

Figure 1. Parametric definition of MDOF systems: (a) frame-type system included in the study; (b) linear added damping system; (c) damper coefficient distribution; (d) column stiffness distribution.

As discussed in Appendix B, values of $\lambda=0.5$ to 2 are usual in steel seismic resisting frames. In this study, six different values of $\lambda(0.10 ; 0.50 ; 1 ; 2 ; 10 ; \infty)$ are considered, with the special case $\lambda_{n}=\infty$ corresponding to so-called shear-type systems, and $\lambda_{n}<\infty$ corresponding to frame-type systems. The vertical distribution of column stiffness is taken as:

$$
\frac{k_{c, e q, n}}{k_{c, e q, 1}}=1+\left(\alpha_{k}-1\right)\left(\frac{n-1}{N-1}\right)^{\beta_{k}},
$$


where the parameter $\alpha_{k}$ represents the proportion between roof and first story stiffness, $\alpha_{k}=k_{c, e q, N} / k_{c, e q, 1}$, and $\beta_{k}$ defines the height-wise variation law of stiffness, see Figure $1 \mathrm{~d}$. A similar concept is used to define the added damping coefficients, see Figure $1 \mathrm{~b}, \mathrm{c}$ :

$$
\frac{c_{d, n}}{c_{d, 1}}=1+\left(\alpha_{d}-1\right)\left(\frac{n-1}{N-1}\right)^{\beta_{d}} .
$$

The frame stiffness matrix is totally defined by $N, k_{c, e q, 1}, \alpha_{k}, \beta_{k}$ and $\lambda$. However, instead of fixing $k_{c, e q, 1}$, this value is determined to target a certain fundamental period $T_{1}$ (an initial value $k_{c, e q, 1}$ is used to assemble the initial stiffness matrix $\mathbf{k}^{*}$ and mass matrix $\mathbf{m}$; using these values the period $T_{1}^{*}$ is calculated, whereupon $k_{c, e q, 1}=k_{c, e q, 1} \cdot T_{1} / T_{1}^{*}$, and the actual stiffness matrix $\mathbf{k}$ can be obtained). The undamped vibrational properties of the system, $\boldsymbol{\Phi}$ and $\omega_{n}$, are calculated from $\mathbf{k}$ and $\mathbf{m}$. The matrix $\mathbf{k}$ is condensed to include only translational terms [17] (pp. 367 ff.). The damped vibrational properties are determined as follows: first, an inherent modal damping ratio $\xi_{h}$ is assumed for all modes, the diagonal inherent modal damping matrix is assembled as $C_{h, j}=2 \xi_{h} \omega_{j} M_{j}$, and the corresponding inherent damping $\mathbf{c}_{h}$ is calculated as:

$$
\mathbf{c}_{h}=\left(\boldsymbol{\Phi}^{T}\right)^{-1} \mathbf{C}_{h} \boldsymbol{\Phi}^{-1}
$$

Second, the added damping matrix $\mathbf{c}_{a}$ is obtained from first principles:

$$
\mathbf{c}_{a}=\mathbf{T}_{U}^{-1} \mathbf{c}_{d} \mathbf{T}_{L}^{-1}
$$

where $\mathbf{T}_{U}$ and $\mathbf{T}_{L}$ are the $N$-dimensional unity upper and lower triangular matrices, respectively, and $\mathbf{c}_{d}=\operatorname{diag}\left(c_{d, n}\right)$ where $\operatorname{diag}\left(x_{n}\right)$ returns a diagonal matrix with $n$-th term $x_{n}$, whereupon the total damping matrix is found as:

$$
\mathbf{c}=\mathbf{c}_{h}+\mathbf{c}_{a},
$$

and the total modal damping matrix is calculated as $\mathbf{C}=\boldsymbol{\Phi}^{T} \mathbf{c} \boldsymbol{\Phi}$. Finally, the approximate modal damping and damping matrices are obtained preserving only the diagonal terms in $\mathrm{C}$ :

$$
\begin{gathered}
\mathbf{C}_{\text {approx }}=\operatorname{diag}\left(C_{n}\right), \\
\mathbf{c}_{\text {approx }}=\left(\boldsymbol{\Phi}^{T}\right)^{-1} \mathbf{C}_{\text {approx }} \boldsymbol{\Phi}^{-1} .
\end{gathered}
$$

It is worth noting that modal damping ratios $\xi_{j}\left(=C_{j} / 2 \omega_{j} M_{j}\right)$ obtained from $\mathbf{C}$ and $\mathbf{C}_{\text {approx }}$ are identical. The added damping matrix $\mathbf{c}_{a}$ is totally defined from $N, c_{d, 1}, \alpha_{d}, \beta_{d}$. However, instead of fixing $c_{d, 1}$, this value is determined to target a certain value of added damping ratio in the first mode $\xi_{a, 1}$ (an initial value $c_{d, 1}{ }^{*}$ is used to assemble the initial modal damping matrix $\mathbf{C}^{*} ; \xi_{a, 1}^{*}$ is then calculated from $C_{1}^{*}$, whereupon $c_{d, 1}=c_{d, 1} * \cdot \xi_{a, 1} / \xi_{a, 1}^{*}$, and the actual added damping matrix $\mathbf{c}_{a}$ can be obtained). $\mathbf{c}_{a}$ is thus a band matrix of bandwidth 1 . This is also the case for $\mathbf{k}$, if $\lambda=\infty$; for other values of $\lambda, \mathbf{k}$ is populated, with increasing off-diagonal terms for lower values of $\lambda$. The formulation used for the damping system assumes pure viscous behavior and thus the influence of auxiliary element flexibility [39] is not included in the study.

As indicated, six different values of $\lambda[0.10 ; 0.50 ; 1 ; 2 ; 10 ; \infty]$ are explored. $\xi_{h}$ is generally taken as 3\%, although some $0 \%$ and $5 \%$ cases are also included to determine the influence of this parameter. With $\lambda$ and $\xi_{h}$ fixed, the remaining parameters defining a damped system are: $N, T_{1}, \xi_{a, 1}, \alpha_{k}, \beta_{k}, \alpha_{d}, \beta_{d}$. The range considered for these 7 parameters in the study is: $N=[3,5,7,9] ; \alpha_{k}=[0.20 ; 0.40 ; 0.60 ; 0.80] ; \beta_{k}=[0.5 ; 1 ; 2 ; 10]$; $\left.\alpha_{d}=[0 ; 1 / 3 ; 2 / 3 ; 1] ; \beta_{d}=[0.1 ; 0.5 ; 1 ; 2]\right) ; T_{1}=[0.5 ; 1 ; 2 ; 4] ; \xi_{a, 1}=[10 \% ; 20 \% ; 30 \% ; 40 \%]$. The values chosen for $N$ and $T_{1}$ cover the most usual cases in building structures equipped with linear viscous dampers, where a certain lateral flexibility is needed to render the 
damping devices useful. The uppermost $40 \%$ value for $\xi_{a, 1}$ is slightly above the current maximum value $\left(\xi_{h, 1}+\xi_{a, 1}=35 \%\right)$ allowed in US practice for application of RSA [8]. The values chosen for $\alpha_{k}$ correspond to realistic possibilities in a design situation; the values chosen for $\alpha_{d}, \beta_{d}$, and $\beta_{k}$ range between extreme $\left(\alpha_{d}=[0,1 / 3], \beta_{d}=0.1, \beta_{k}=10\right)$ and usual $\left(\alpha_{d}=[2 / 3,1], \beta_{d}=[0.5,1,2], \beta_{k}=[0.5,1,2]\right)$. The extreme value $\left(\alpha_{d}=0, \beta_{d}=0.1\right)$ represents approximately a structure with additional dampers only in the first story. For $\alpha_{d}=1$ any value of $\beta_{d}$ results in the same damping system, thus only $\beta_{d}=2$ need to be considered, and $3 \cdot 4^{5}$ repeated cases $\left(\alpha_{d}=1\right.$ with $\beta_{d}=0.1,0.5$ and 1$)$ are eliminated from the set. The total number of different systems examined for every combination of $\lambda$ and $\xi_{h}$ is therefore $13,312\left(4^{7}-3 \cdot 4^{5}\right)$. Combinations of $\alpha_{k}, \beta_{k}, \alpha_{d}$ and $\beta_{d}$ considered in the study are illustrated in Figure 2.
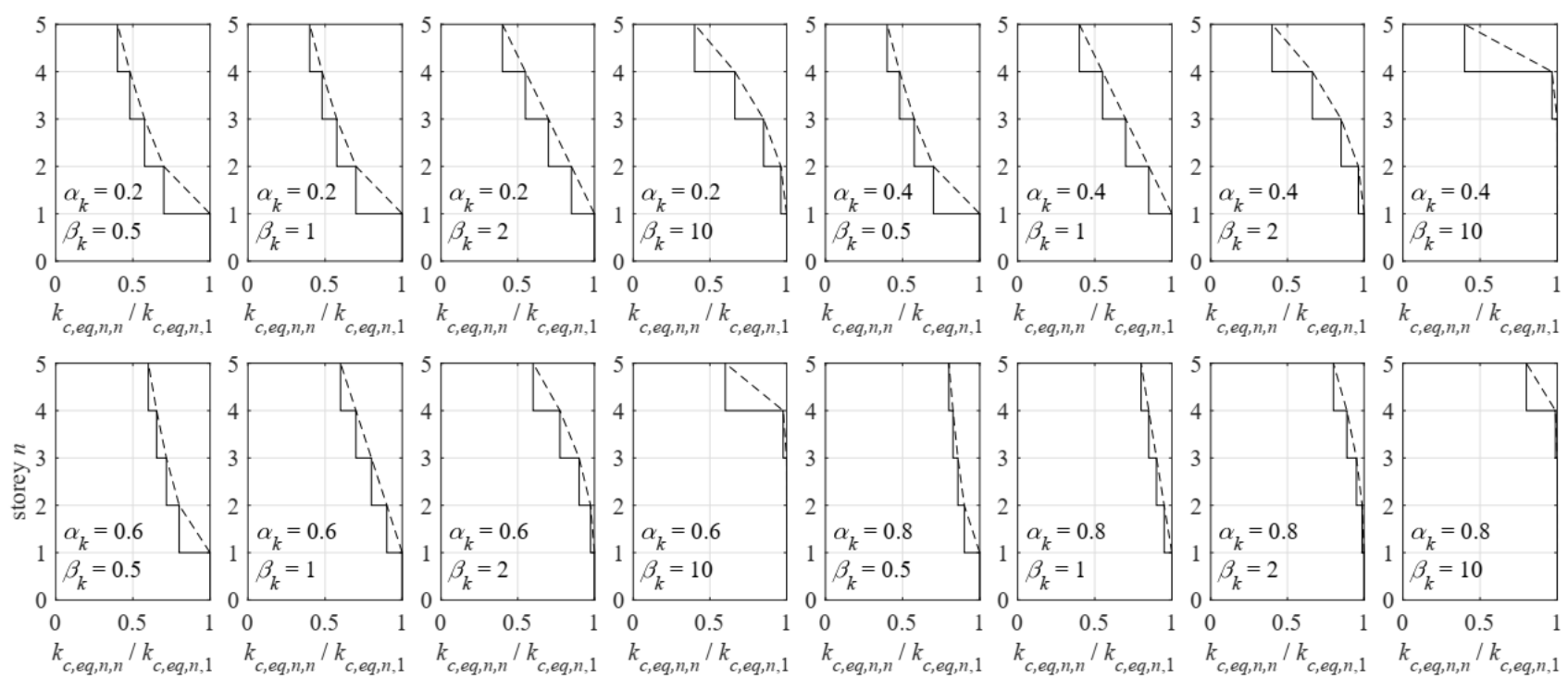

(a)
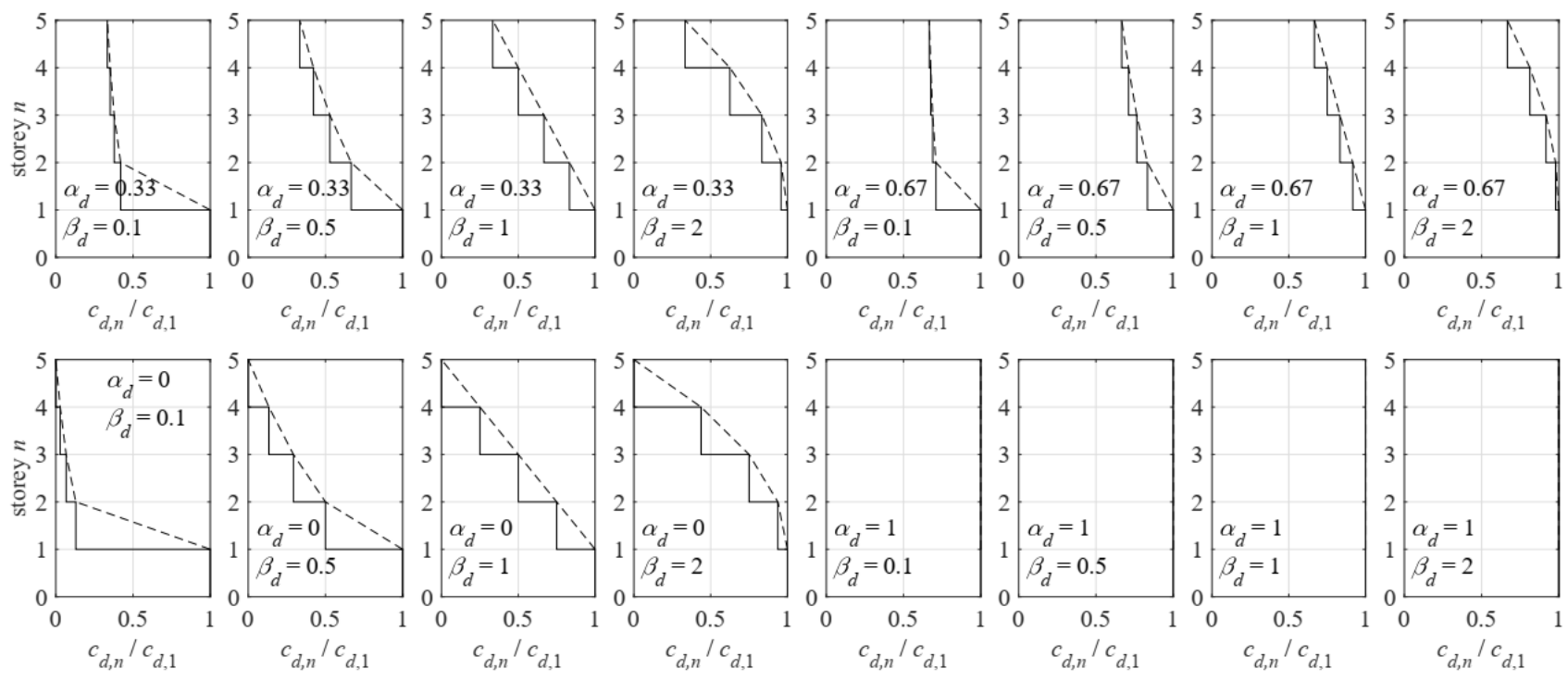

(b)

Figure 2. Vertical distributions considered in the study; (a) stiffness; (b) added damping. 


\subsubsection{Ground Motions}

40 far-field records are chosen from the European Strong Motion Database [40,41] and binned according to site class B (20 records) and C (20 records). These site classes were adopted as defined in the European code (Eurocode 8) [42] and represent firm (B) to medium (C) ground type conditions. Both horizontal components from each record are consistently included and treated as independent registers, adding up a total of $N R=40$ accelerograms per ground type. The accelerograms corresponding to each ground type are normalized and pre-scaled so that their mean acceleration spectrum represents well the $5 \%$ Eurocode 8 normalized pseudo-acceleration spectrum at $P H A=0.3 \mathrm{~g}$, in a wide range of periods from $T_{C}$ to $T_{D}$ (code-defined spectral corner periods for each site class); within this range the mean spectral value of the set is always above $90 \%$ of the code spectral value. This collection of scaled accelerograms is referred to as a Ground Motion Set (GMS). The selection of motions is listed in Table A2 (GMS B) and Table A3 (GMS C) of Appendix C, where Figure A2 shows the pre-scaled normalized 5\% elastic spectra for both sets.

\subsubsection{Cases Examined}

In this study a case is formed by a GMS (ground motion set), a value of inherent damping $\xi_{h}$, and a value of beam to column relative stiffness $\lambda$. The case is formed by the 13,312 systems formed by combinations of the 7 remaining parameters $N, T_{1}, \xi_{a, 1}, \alpha_{k}, \beta_{k}, \alpha_{d}$ and $\beta_{d}$. The cases are referenced by labels such as 'B3-1', where 'B' refers to the GMS, ' 3 ' is the value of $\xi_{h}$ (expressed in \%) and '1' is the value of $\lambda$ (in shear-type systems $\lambda=\infty$ ). The following cases are considered:

- $\quad$ Shear-type systems: B0- $\infty$, B3- $\infty$, B5- $\infty, \mathrm{C} 0-\infty, \mathrm{C} 3-\infty, \mathrm{C} 5-\infty$.

- $\quad$ Frame-type systems: B3-0.1, B3-0.5, B3-1, B3-2, B3-10, B0-1, B5-1, C3-1.

\subsubsection{Procedures and Response Variables}

For a given system $n s$ included in case $n c$, three different procedures are used to find the solution for each register $r$ in the GMS. These procedures are described in Table 1, where the notation used in the study is introduced.

The procedures listed in Table 1 (TH, THMA, RSA-SRSS and RSA-CQC), are described hereby:

(1) Time-History (TH): the complete response ${ }_{n s}^{r} \mathbf{u}(t),{ }_{n s}^{r} \mathbf{v}(t),{ }_{n s}^{r} \mathbf{a}(t)$ (relative displacement, relative velocity, and absolute acceleration, respectively) of the system defined by ${ }_{n s} \mathbf{m},{ }_{n s} \mathbf{k}$ and ${ }_{n s} \mathbf{c}$ (non-classically damped system) to each scaled register $r$ in the GMS is calculated by the central difference step-by-step method [14]. The corresponding restoring forces ${ }_{n s}^{r} \mathbf{f}_{\mathrm{S}}(t)$ and damping forces ${ }_{n s}^{r} \mathbf{f}_{\mathrm{D}}(t)$ are obtained as ${ }_{n s}^{r} \mathbf{k} \cdot{ }_{n s}^{r} \mathbf{u}(t)$ and ${ }_{n s}^{r} \mathbf{c} \cdot{ }_{n s}^{r} \mathbf{v}(t)$, respectively. Other variables are derived from these. The peak response for each variable and story is preserved.

(2) Time-history modal analysis (THMA): the complete response ${ }_{n s}^{r} \mathbf{u}_{1}(t),{ }_{n s}^{r} \mathbf{v}_{1}(t),{ }_{n s}^{r} \mathbf{a}_{1}(t)$ of the system defined by ${ }_{n s} \mathbf{m},{ }_{n s} \mathbf{k}$ and ${ }_{n s} \mathbf{c}_{\text {approx }}$ (approximate classically damped system) to each scaled register $r$ in the GMS, are calculated adding the responses obtained for each mode at each instant by the central difference step-by-step method. As in the previous procedure, restoring forces ${ }_{n s}^{r} \mathbf{f}_{\mathrm{S} 1}(t)$ and damping forces ${ }_{n s}^{r} \mathbf{f}_{\mathrm{D} 1}(t)$ are obtained as ${ }_{n s}^{r} \mathbf{k} \cdot{ }_{n s}^{r} \mathbf{u}_{1}(t)$ and ${ }_{n s}^{r} \mathbf{c} \cdot{ }_{n s}^{r} \mathbf{v}_{1}(t)$, respectively; the forces obtained for every time-step are not in equilibrium, because ${ }_{n s}^{r} \mathbf{f}_{\mathrm{D} 1}(t)$ is calculated using ${ }_{n s} \mathbf{c}$ whereas ${ }_{n s}^{r} \mathbf{u}_{1}(t)$ and ${ }_{n s}^{r} \mathbf{v}_{1}(t)$ are calculated using ${ }_{n s} \mathbf{c}_{\text {approx }}$, as is usual in design applications. Peak responses for every variable and story are preserved. THMA avoids the inaccuracies involved in the use of approximate modal combination rules, therefore only the first type of error discussed in Section 2.1 (due to neglected off-diagonal terms of the modal damping matrix) is present; this procedure is identified by subscript ' 1 '.

(3) Response spectrum analysis (RSA-SRSS and RSA-CQC): for every mode $j$ of system $n s$ defined by ${ }_{n s} \mathbf{m},{ }_{n s} \mathbf{k}$ and ${ }_{n s} \mathbf{c}_{\text {approx }}$ (approximate classically damped system), and for every register $r$ in the GMS, the modal peak relative displacement ${ }_{n s}^{r} \mathbf{u}_{2, \text { peak,j, }}$, modal peak relative velocity ${ }_{n s}^{r} \mathbf{v}_{2, \text { peak,j }}$, and modal peak absolute acceleration ${ }_{n s}^{r} \mathbf{a}_{2, \text { peak,j, }}$, are found 
using the piecewise step-by-step method [43]. These vectors contain the peak values for every story at mode $j$, for example ${ }_{n s}^{r} \mathbf{u}_{2, \text { peak }, j}=\left[{ }_{n s}^{r} u_{2, \text { peak }, 1, j} \ldots, r{ }_{n s}^{r} u_{2, \text { peak }, N, j}\right]^{T}$, etc. The modal peak values of restoring forces ${ }_{n s}^{r} \mathbf{f}_{\mathrm{S} 2, \text { peak,j }}\left(={ }_{n s}^{r} \mathbf{k} \cdot{ }_{n s}^{r} \mathbf{u}_{2, \text { peak,j }}\right)$ and damping forces ${ }_{n s}^{r} \mathbf{f}_{\mathrm{D} 2 \text {, peak,j }}\left(={ }_{n s}^{r} \mathbf{c}_{n s}^{r} \mathbf{v}_{2, \text { peak, }, j}\right)$ are found. The modal peak values of other variables are derived from these. The peak response for every variable and story is obtained applying the SRSS rule (RSA-SRSS), identified with subscript ' 2 ', or CQC rule (RSA-CQC), identified with subscript ' 3 '. This option corresponds closely with the Response Spectrum Procedure defined in Chapter 18 of ASCE-SEI 7/16 [8], except that here it is applied only to linear systems. The SRSS [19] and CQC [20,21] rules are defined hereby for completeness:

$$
\begin{gathered}
\operatorname{SRSS}\left(\mathbf{x}_{j}\right)=\sqrt{\sum_{j=1}^{N} x_{n j^{\prime}}^{2}} \\
\operatorname{CQC}\left(\mathbf{x}_{j}, \mathbf{T}, \xi\right)=\sqrt{\sum_{i=1}^{N} \sum_{j=1}^{N} x_{n i} r_{i j} x_{n j},} \\
r_{i j}=\frac{8 \sqrt{\xi_{i} \xi_{j}}\left(\xi_{i}+\rho_{i j} \xi_{j}\right) \rho_{i j}^{3 / 2}}{\left(1-\rho_{i j}^{2}\right)^{2}+4 \xi_{i} \xi_{j} \rho_{i j}\left(1+\rho_{i j}^{2}\right)+4\left(\xi_{i}^{2}+\xi_{j}^{2}\right) \rho_{i j}^{2}},
\end{gathered}
$$

where $\mathbf{x}_{j}=\left[x_{1 j}, \ldots, x_{N j}\right]^{T}$ is the variable response for mode $j, \mathbf{T}=\left[T_{1}, \ldots, T_{N}\right]^{T}$,

\begin{tabular}{|c|c|c|c|}
\hline Procedure & TH & THMA & RSA-SRSS $(* *)$ \\
\hline Description. & $\begin{array}{c}\text { Time history } \\
\text { step-by-step analysis }\end{array}$ & Time history modal analysis & Response spectrum analysis \\
\hline System $n s$ definition & ${ }_{n s} \mathbf{m},{ }_{n s} \mathbf{k},{ }_{n s} \mathbf{c}$ & ${ }_{n s} \mathbf{m},{ }_{n s} \mathbf{k},{ }_{n s} \mathbf{c}_{\text {approx }}$ & ${ }_{n s} \mathbf{m},{ }_{n s} \mathbf{k},{ }_{n s} \mathbf{C}_{\text {approx }}$ \\
\hline Analysis method. & $\begin{array}{l}\text { Central difference applied to } \\
\text { complete system. }\end{array}$ & $\begin{array}{l}\text { Central difference applied to } \\
\text { complete system. }\end{array}$ & $\begin{array}{l}\text { Exact piecewise applied } \\
\text { to each mode. }\end{array}$ \\
\hline $\begin{array}{l}\text { Solution type (for } \\
\text { register } r \text { in case } n c \text { ). }\end{array}$ & $\underset{n s}{r} \mathbf{C}(t),{ }_{n s}^{r} \mathbf{v}(t),{ }_{n s}^{r} \mathbf{a}(t)$ & $\begin{array}{l}\text { Complete solution. } \\
{ }_{n s}^{r} \mathbf{u}_{1}(t),{ }_{n s}^{r} \mathbf{v}_{1}(t),{ }_{n s}^{r} \mathbf{a}_{1}(t)\end{array}$ & $\begin{array}{l}\text { Peak modal solution (mode } j \text { ). } \\
\underset{n s}{r} \mathbf{u}_{2, \text { peak,j, }}{ }_{n s}^{r} \mathbf{v}_{2, \text { peak }, j},{ }_{n s} \mathbf{a}_{2, \text { peak }, j}\end{array}$ \\
\hline $\begin{array}{l}\text { Total damping forces (for } \\
\text { register } r \text { in case } n c \text { ). }\end{array}$ & ${ }_{n s}^{r} \mathbf{f}_{\mathrm{D}}(t)=\mathbf{c} \cdot{ }_{n s}^{r} \mathbf{v}(t)$ & ${ }_{n s}^{r} \mathbf{f}_{\mathrm{D} 1}(t)=\mathbf{c} \cdot{ }_{n s}^{r} \mathbf{v}_{1}(t)$ & ${ }_{n s}^{r} \mathbf{f}_{\mathrm{D} 2, \text { peak }, j}(t)=\mathbf{c} \cdot{ }_{n s}^{r} \mathbf{v}_{2, \text { peak }, j}(t)$. \\
\hline $\begin{array}{l}\text { Variables derived from solution } \\
\quad \text { (for register } r \text { in case } n c) \text {. }\end{array}$ & $\begin{array}{l}{ }_{n s}^{r} \Delta(t),{ }_{n s}^{r} \nabla(t),{ }_{n s}^{r} \mathbf{f}_{\mathrm{S}}(t), \\
{ }_{n s}^{r} \mathbf{f}_{\mathrm{D}}(t),{ }_{n s}^{r} \mathbf{f}_{\mathrm{T}}(t),{ }_{n s}^{r} \mathbf{V}_{\mathrm{S}}(t), \\
{ }_{n s}^{r} \mathbf{V}_{\mathrm{D}}(t),{ }_{n s}^{r} \mathbf{V}_{\mathrm{T}}(t) .\end{array}$ & $\begin{array}{c}{ }_{n s}^{r} \Delta_{1}(t),{ }_{n s}^{r} \nabla_{1}(t),{ }_{n s}^{r} \mathbf{f}_{\mathrm{S} 1}(t), \\
{ }_{n s}^{r} \mathbf{f}_{\mathrm{D} 1}(t),{ }_{n s}^{r} \mathbf{f}_{\mathrm{T} 1}(t),{ }_{n s}^{r} \mathbf{V}_{\mathrm{S} 1}(t), \\
r{ }_{n s} \mathbf{V}_{\mathrm{D} 1}(t),{ }_{n s}^{r} \mathbf{V}_{\mathrm{T} 1}(t) .\end{array}$ & 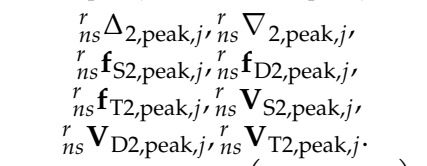 \\
\hline $\begin{array}{l}\text { Peak solution } \\
\text { (for register } r \text { in case } \\
n c \text { and story } s \text { ). }\end{array}$ & $\begin{array}{l}{ }_{n s}^{r} u_{\text {peak,s }}=\left|{ }_{n s}^{r} u_{s}(t)\right|_{\max } . \\
\text { Similar for other variables. }\end{array}$ & $\begin{array}{l}{ }_{n s}^{r} u_{1, \text { peak }, s}=\left|{ }_{n s}^{r} u_{1, s}(t)\right|_{\max } \cdot \\
\text { Similar for other variables. }\end{array}$ & $\begin{array}{l}{ }_{n s}^{r} u_{2, \text { peak,s }}=\operatorname{SRSS}\left({ }_{n s}^{r} u_{2, \text { peak,s, }}\right) . \\
\text { Similar for other variables }\left(^{* *}\right) .\end{array}$ \\
\hline $\begin{array}{l}\text { Error for register } r \text { in case } \\
n c \text { and story } s .\end{array}$ & Does not apply & ${ }_{n s}^{r} u_{1, \text { error }, s}={ }_{n=}^{r} u_{1, \text { peak }, s}^{r}-1$. & 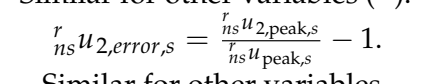 \\
\hline Significant stories considered. & Does not apply & $\begin{array}{c}\text { Similar for other variables. } \\
\text { First: }{ }_{n s}^{r} u_{1, \text { error,first }} \\
\text { Central: }{ }_{n s} u_{1, \text { error,central }} \\
\text { Roof: }{ }_{n s}^{r} u_{1, \text { error,roof }} \\
\text { Average: }{ }_{n s}^{r} u_{1, \text { error,average }}= \\
=\sum_{s=1}^{N}{ }_{n s}^{r} u_{1, \text { error, }, s} / N . \\
\text { Similar for other variables. }\end{array}$ & $\begin{array}{c}\text { Similar for other variables. } \\
\text { First: }{ }_{n s}^{r} u_{2, \text { error,first }} \\
\text { Central: }{ }_{n s}^{r} u_{2, \text { error,central }} \\
\text { Roof: }{ }_{n s}^{r} u_{2, \text { error,roof }} \\
\text { Average: }{ }_{n s}^{r} u_{2, \text { error,average }}= \\
=\sum_{s=1}^{N}{ }_{n s}^{r} u_{2, \text { error, }} / N . \\
\text { Similar for other variables. }\end{array}$ \\
\hline $\begin{array}{l}\text { Final error for system } n s \text { and } \\
\text { case } n c \text { at story } s .\end{array}$ & Does not apply & $\begin{array}{l}\quad n_{s} u_{1, \text { error }, s}= \\
\quad=\sum_{r=1}^{N} n_{s} u_{1, \text { error, } s} / N R, \\
\text { where } s=\text { first, central, average, } \\
\text { roof.Similar for other variables. }\end{array}$ & $\begin{array}{l}\quad n s u_{2, \mathrm{error}, s}= \\
\quad=\sum_{r=1 r n s}^{N} u_{2, \mathrm{error}, s} / N R, \\
\text { where } s=\text { first, central, average, } \\
\text { roof.Similar for other variables }\end{array}$ \\
\hline
\end{tabular}
$\xi=\left[\xi_{1}, \ldots, \xi_{N}\right]^{T}$, and $\rho_{i j}=T_{i} / T_{j}$.

Table 1. Summary of procedures and notation used in the numerical study.

Notes: peak = maxima of unsigned response. Variables: $\mathbf{u}=$ relative displacement; $\mathbf{v}=$ relative velocity; $\mathbf{a}=$ total acceleration; $\boldsymbol{\Delta}$ inter-story drift; $\boldsymbol{\nabla}$ inter-story drift velocity; $\mathbf{f}_{S}$ restoring structural force; $\mathbf{f}_{\mathrm{D}}$ damping force; $\mathbf{f}_{\mathrm{T}}$ total force; vs. restoring structural shear; $\mathbf{V}_{\mathrm{D}}$ damping shear; $\mathbf{V}_{\mathrm{T}}$ total shear; $N$ : total number of stories; NR: number of registers in set. $\left.{ }^{* *}\right)$ RSA-CQC is found with the same procedure as RSA-SRSS substituting SRSS (.) by CQC(·) at peak solution calculation, and replacing subscript ' 2 ' by ' 3 '. 
The response variables of interest are story displacement $u$, story velocity $v$, inter-story drift $\Delta$ (hereinafter referred to as drift), inter-story drift velocity $\nabla$ (hereinafter referred to as drift velocity); total (or absolute) acceleration $a$; structural shear force $V_{\mathrm{S}}$; damper shear force $V_{\mathrm{D}}$ (calculated with the exact damping matrix); total shear force $V_{\mathrm{T}}$ (addition of $V_{\mathrm{D}}$ and $V_{\mathrm{T}}$ ). As indicated in Table 1, variables obtained from THMA, RSA-SRSS and RSA-CQC are marked off by adding subscripts 1, 2 or 3, respectively. The stories chosen as representative are: $N^{\text {th }}$ story (referred to as 'roof'); 1st story (referred to as 'first story' or simply 'first'); central story $(N+1) / 2$ (referred to as 'central'); finally, the mean value throughout stories (referred to as 'average story' or simply 'average') is also found.

\subsubsection{Measure of Response Errors}

The deviation between TH results and approximate results (THMA, RSA) is referred to as 'error', and measured for every register $r$ in case $n c$, response variable, story $s$ and system $n s$. For instance, for $\operatorname{drift}(\Delta$ and story $s$ of system $n s$ in register $r$ of the case:

$$
{ }_{n s}^{r} \Delta_{1, \text { error }, s}=\frac{{ }_{n s}^{r} \Delta_{1, \text { peak }, s}}{{ }_{n s}^{r} \Delta_{\text {peak }, s}}-1,
$$

where $s$ can be 'first', 'central', 'average' or 'roof'. Similar equations (with updated subscripts) are used for RSA-SRSS $\left({ }_{n s}^{r} \Delta_{2, \text { error,s }}\right)$ and RSA-CQC $\left({ }_{n s}^{r} \Delta_{3, \text { error,s }}\right)$. A negative value of the error so defined indicates that the approximate method underestimates the response (unsafe situation), whereas a positive value indicates that the approximate method overestimates the response (safe situation). The mean value across the $N R$ registers in case $n c$ is considered as significant for the system $n s$; for instance, for drift $\Delta$ and story $s$ :

$$
{ }_{n s} \Delta_{1, \mathrm{error}, \mathrm{s}}=\frac{\sum_{r=1{ }_{n s} \Delta_{1, \mathrm{error}, \mathrm{s}}}^{N R} .}{N R}
$$

A similar definition is used for other variables. Finally, statistical summaries across $n s$ are obtained. In this study the focus is placed on the $10 \%, 50 \%$ and $90 \%$ percentiles $\left(x^{10}\right.$, $\left.x^{50}, x^{90}\right)$, and extreme values, minimum ( $\left.\min \right)$ and maximum (max).

\section{Results}

Figure 3 displays a comparison of height-wise distribution for THMA error (error 1) and six relevant variables $\left(u_{1}, \Delta_{1}, \nabla_{1}, a_{1}, V_{\mathrm{S} 1}, V_{\mathrm{D} 1}\right)$ in two representative systems (B3-1, typical of frame behavior and B3- $\infty$, representative of pure shear behavior for GMS B and $3 \%$ inherent damping). Every sub-plot displays the $\min , x^{10}, x^{50}, x^{90}$ and max error for each story, thus allowing quick identification of the error magnitude and vertical distribution. For brevity, similar plots for RSA-SRSS error (error 2) and RSA-CQC error (error 3) are included as Supplementary Materials, Figures S1 and S2.

This information is supplemented by scatter plots providing a visual overview of THMA error (error 1) in Figures 4 and 5. In addition to sheer representation of the data points, useful analytical results are also provided in the last set of plots: (i) three markers have been included pointing the $10 \%, 50 \%$, and $90 \%$ percentiles $\left(x^{10}, x^{50}, x^{90}\right)$ of data in the set (triangular red, circular blue and triangular green markers, respectively), and the variable values corresponding to these percentiles are indicated in the left and bottom margins in the corresponding color; (ii) an Engineering Acceptability Range (EAR) [ $-0.15 ;+0.25]$ has been marked with dashed lines (red and green) as a visual reference to allow quick comparison between plots; the percentage of data below, within and above this range is indicated at the right and top margins of the plot; (iii) the maximum and minimum value of each data set is presented at the bottom right corner (for data in y axis) and top left corner (for data in $x$ axis); (iv) the Pearson correlation coefficient $\rho$ between both sets of data is presented at the bottom left corner of the plot. To simplify interpretation, text regarding data for $x$-axis is oriented vertically, whereas text regarding data for $y$-axis is oriented horizontally; the correlation coefficient text is rotated diagonally. As an example, 


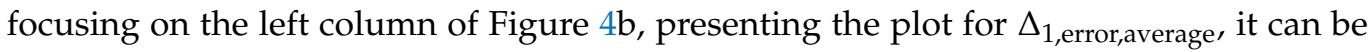
seen that for the case B3-1 100\% results are within EAR, with $x^{50}=-0.01, x^{10}=-0.05$ and $x^{90}=+0.01$, and extreme values $x_{\min }=-0.16, x_{\max }=+0.20$; for case B3- $\infty, 4.3 \%$ of the results are too unconservative (below -0.15 ) and $95.7 \%$ of results are within range, with $x^{50}=-0.01, x^{10}=-0.10, x^{90}=+0.02$ and extreme values $x_{\min }=-0.28$ and $x_{\max }=+0.26$. Between both sets the correlation is $\rho=0.76$. The Engineering Acceptability Range (EAR) was introduced by Guyader and Iwan in the context of equivalent linearization to express the acceptable range of error when an engineering variable is obtained using an approximate method [44]. The asymmetry of the range follows from the fact that conservative errors are, engineeringly speaking, more acceptable than unconservative ones. The range adopted in this study is based on a survey among structural engineering professionals and is purely indicative; details can be found in [25].
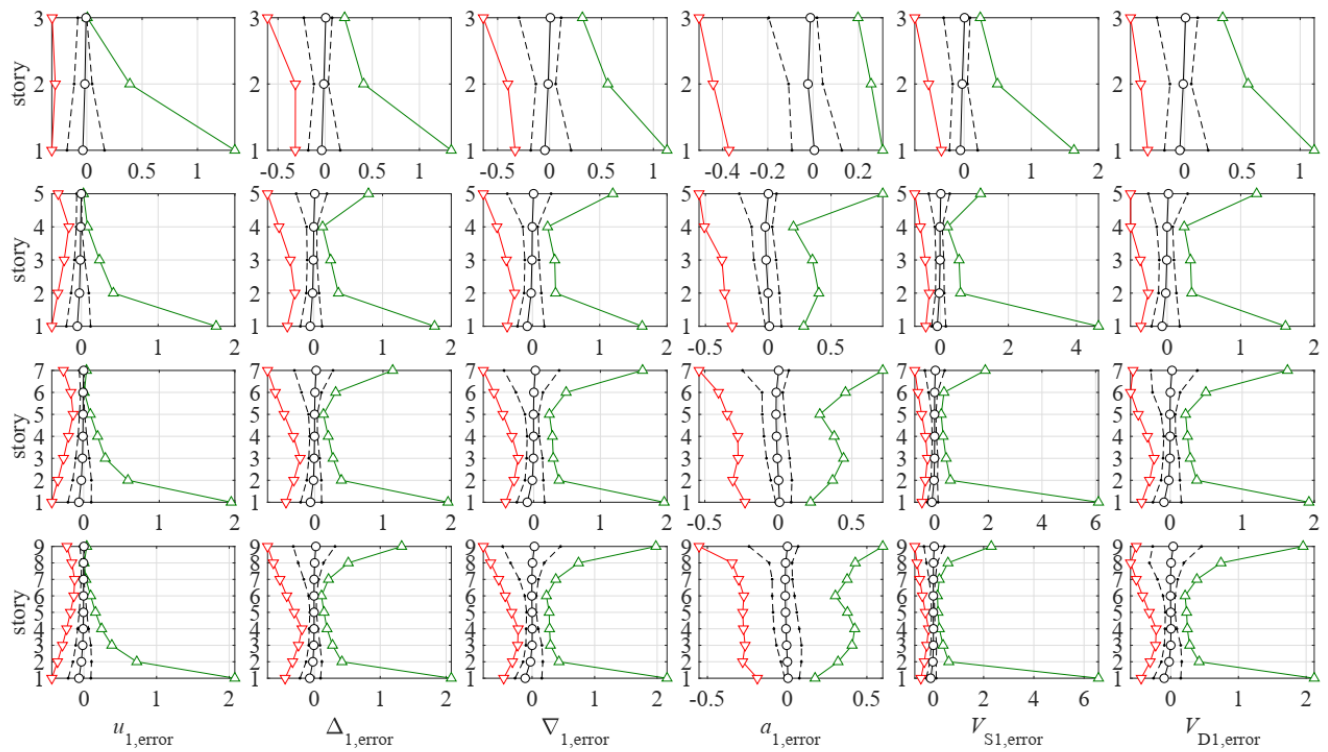

(a)
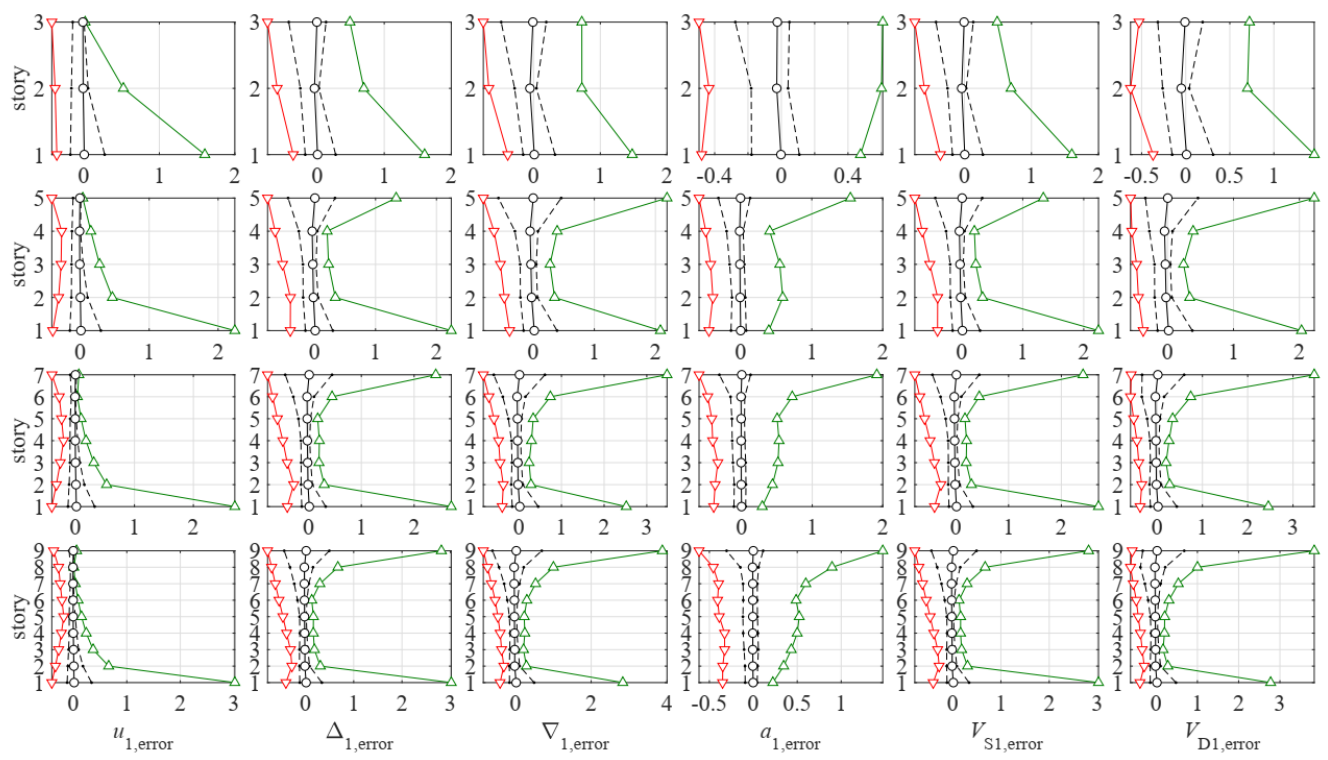

(b)

$\nabla \min \cdots 10 \% \cdot-50 \% \cdots 90 \% \triangleleft \max$

Figure 3. Plots of THMA errors (error 1): (a) frame-type system B3-1; (b) shear-type system B3- $\infty$. 
A comprehensive overview of results (including several cases) for THMA (error 1) at average of stories, first story and roof) is offered in Figure A3 $\left(u_{1}\right.$ and $\left.\Delta_{1}\right)$, Figure A4 $\left(\nabla_{1}\right.$ and $\left.a_{1}\right)$ and Figure A5 $\left(V_{\mathrm{S} 1}, V_{\mathrm{D} 1}\right)$ included in Appendix D; in these figures for every case, variable and story: (i) a modified whisker-and-box diagram (referred hereinafter as bar plot) is produced, where the $x$-axis represents the value of the error and the extension of the base line is indicative of the full range of error results; (ii) a thick line indicates the range comprised between $10 \%$ and $90 \%$ percentiles, with the median indicated by a circular hollow marker; (iii) for each case, numerical values are indicated for the maximum (green, labeled max) and minimum (red, labeled min) errors, as well as for the $10 \%, 50 \%$ and $90 \%$ percentiles; iv) an indicative EAR is marked by vertical dash-dot lines at errors -0.15 (red) and +0.25 (blue), to help in visual comparison of the plot horizontal scale. As an example, focusing in Figure A3a, and case B3-1, the min, 10\%, 50\%, 90\% and max errors, re-

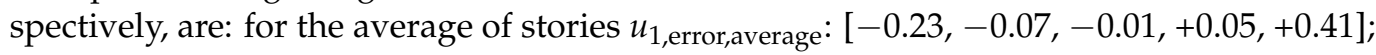
for the first story $u_{1, \text { error,first }}:[-0.44,-0.17,-0.04,+0.17,+2.08]$; for the roof $u_{1, \text { error,roof: }}$ : $[-0.31,-0.08,-0.00,+0.01,+0.04]$. For brevity, similar plots produced for RSA-SRSS (error 2 ) and RSA-CQC (error 3) for all relevant variables, are included as Supplementary Materials: Figure S3 $(u)$; Figure S4 $(v)$; Figure S5 $(\Delta)$; Figure S6 $(\nabla)$; Figure S7 $(a)$; Figure S8 $\left(V_{\mathrm{S}}\right)$; and Figure $\mathrm{S} 9\left(V_{\mathrm{D}}\right)$. Additional scatter plots relating the first story THMA error with the roof THMA error for case B3-1 are given in Figure 6. Finally, a numerical summary of results is given in Table 2 (frame-type systems, B0-1, B3-1, B5-1, C3-1, B3-0.5, B3-2) and Table 3 (shear-type systems, B0- $\infty$, B3- $\infty$, B5- $\infty, \mathrm{C} 0-\infty, \mathrm{C} 3-\infty, \mathrm{C} 5-\infty$ ), where the mean $\mu$ and standard deviation $\sigma$ of five statistics ( $\min , x^{10}, x^{50}, x^{90}$, max) across cases is calculated for every variable and story. For instance, in Table 2 , the mean $(\mu)$ and standard deviation $(\sigma)$ for variable $u_{1}$ corresponding to first story and $x^{10}$ are $\mu=-0.17, \sigma=0.01$, obtained as statistics of 6 values of $x^{10}[-0.18,-0.17,-0.16,-0.17,-0.17,-0.16]$, each value corresponding to one of the six frame-type systems (B0-1, B3-1, B5-1, C3-1, B3-0.5, B3-2) as shown in Figure A3a for variable $u_{1, \text { error,first }}$.
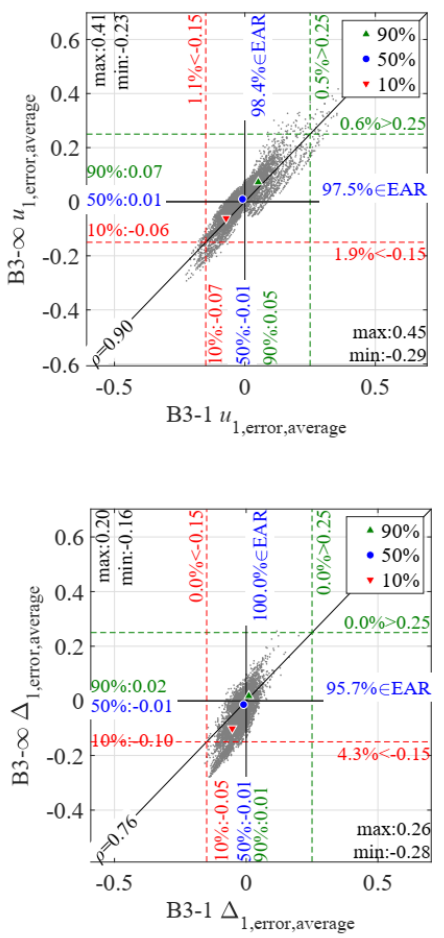

Figure 4. Cont.

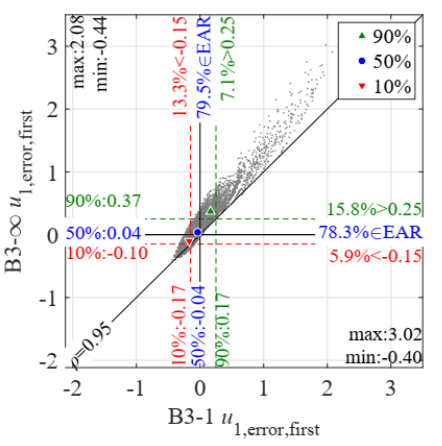

(a)
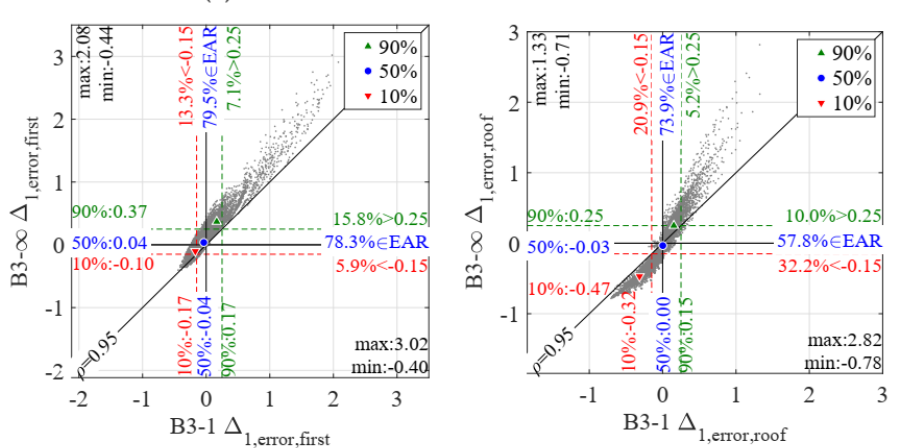

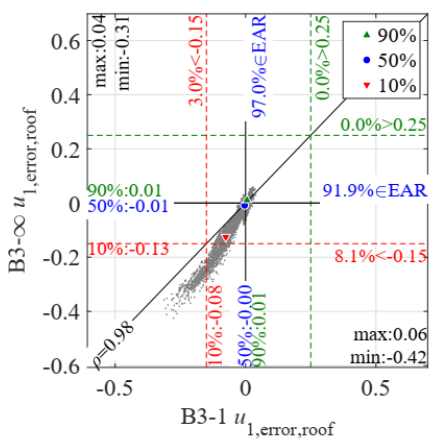

(b) 

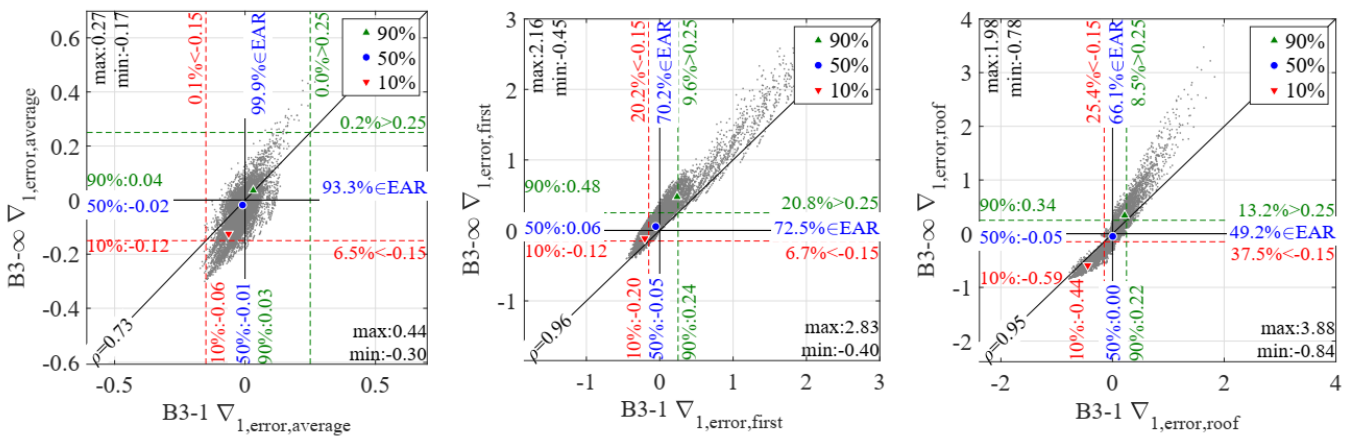

(c)

Figure 4. Scatter plots of case B3-1 versus case B3- $\infty$ at average of stories (left column), first story (central column) and roof (right column) for kinematic variables: (a) relative displacement $u$; (b) inter-story drift $\Delta$; (c) inter-story drift velocity $\nabla$.
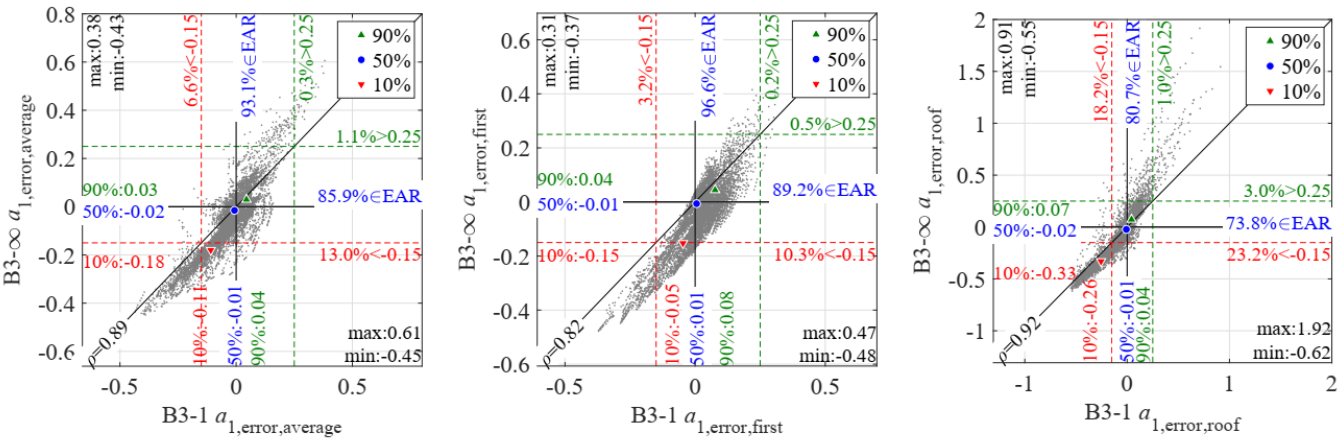

(a)
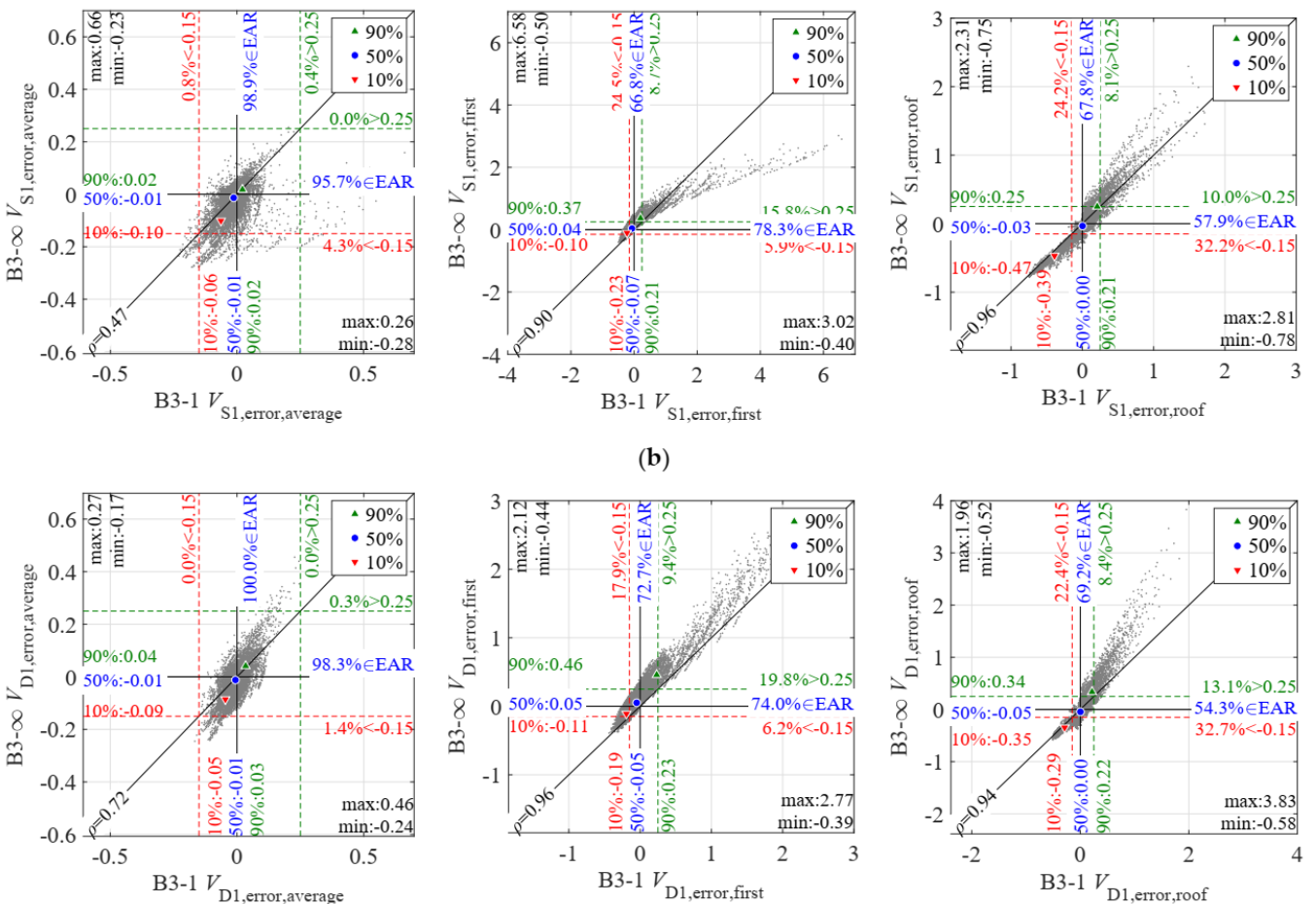

(c)

Figure 5. Scatter plots of case B3-1 versus case B3- $\infty$ at average of stories (left column), first story (central column) and roof (right column) for static variables: (a) absolute acceleration $a$; (b) story shear $V_{\mathrm{S}}$; (c) damping shear $V_{\mathrm{D}}$. 


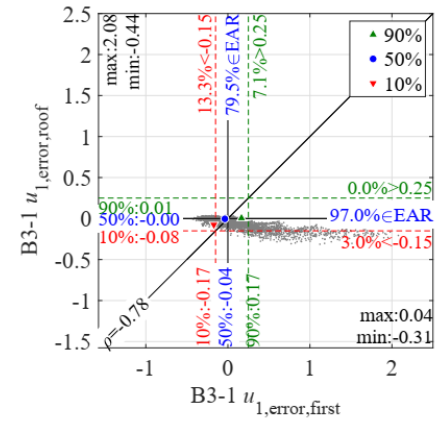

(a)

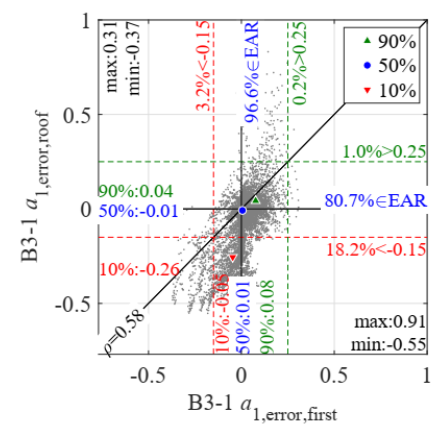

(d)

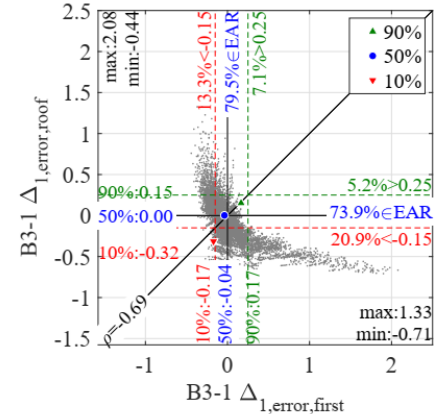

(b)

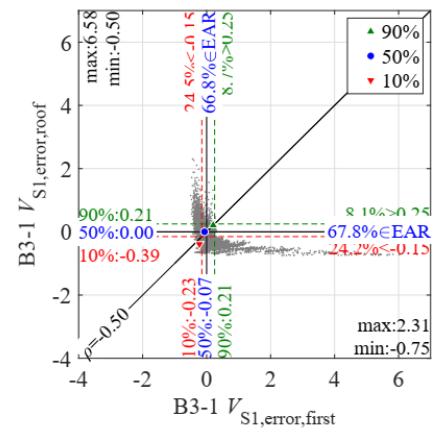

(e)

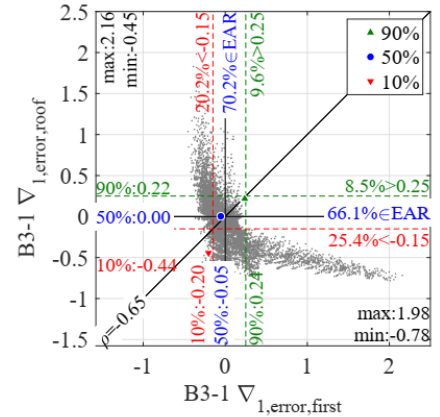

(c)

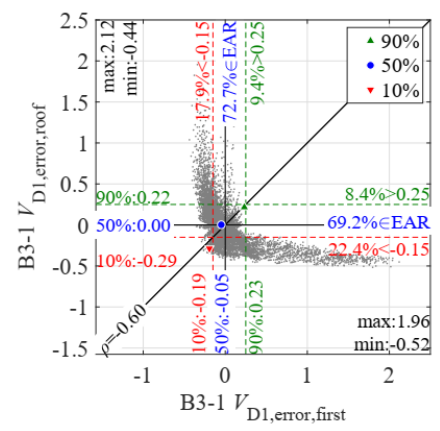

(f)

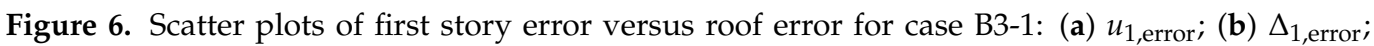
(c) $\nabla_{1, \text { error }} ;$ (d) $a_{1, \text { error }} ;$ (e) $V_{\mathrm{S} 1 \text {,error }}$ (f) $V_{\mathrm{D} 1 \text {,error }}$.

Table 2. Frame-type cases (B0-1, B3-1, B5-1, C3-1, B3-0.5, B3-2), mean $\mu$ and standard deviation $\sigma$ of error across cases.

\begin{tabular}{|c|c|c|c|c|c|c|c|c|c|c|c|c|c|c|c|c|}
\hline & & \multicolumn{5}{|c|}{ First Story } & \multicolumn{5}{|c|}{ Average Across Stories } & \multicolumn{5}{|c|}{ Roof } \\
\hline & & $\min$ & $x^{10}$ & $x^{50}$ & $x^{90}$ & $\max$ & $\min$ & $x^{10}$ & $x^{50}$ & $x^{90}$ & $\max$ & $\min$ & $x^{10}$ & $x^{50}$ & $x^{90}$ & $\max$ \\
\hline \multirow{8}{*}{$\mu$} & $u_{1}$ & -0.44 & -0.17 & -0.04 & 0.16 & 2.05 & -0.23 & -0.07 & -0.01 & 0.05 & 0.40 & -0.32 & -0.08 & 0.00 & 0.01 & 0.04 \\
\hline & $v_{1}$ & -0.46 & -0.20 & -0.05 & 0.24 & 2.17 & -0.24 & -0.08 & -0.01 & 0.08 & 0.38 & -0.35 & -0.09 & -0.01 & 0.01 & 0.05 \\
\hline & $a_{1}$ & -0.37 & -0.05 & 0.01 & 0.08 & 0.31 & -0.44 & -0.11 & -0.01 & 0.04 & 0.37 & -0.56 & -0.26 & -0.01 & 0.04 & 0.88 \\
\hline & $\Delta_{1}$ & -0.44 & -0.17 & -0.04 & 0.16 & 2.05 & -0.16 & -0.05 & -0.01 & 0.01 & 0.19 & -0.71 & -0.31 & 0.00 & 0.15 & 1.35 \\
\hline & $\nabla_{1}^{1}$ & -0.46 & -0.20 & -0.05 & 0.24 & 2.17 & -0.17 & -0.06 & -0.01 & 0.03 & 0.26 & -0.78 & -0.44 & 0.00 & 0.22 & 1.98 \\
\hline & $V_{\mathrm{S} 1}^{1}$ & -0.49 & -0.22 & -0.06 & 0.20 & 6.00 & -0.24 & -0.07 & -0.01 & 0.02 & 0.65 & -0.75 & -0.39 & 0.00 & 0.20 & 2.31 \\
\hline & $V_{\mathrm{D} 1}^{\mathrm{S1}}$ & -0.44 & -0.19 & -0.05 & 0.23 & 2.14 & -0.18 & -0.05 & -0.01 & 0.04 & 0.29 & -0.55 & -0.25 & 0.01 & 0.23 & 2.05 \\
\hline & $u_{1}$ & 0.01 & 0.01 & 0.01 & 0.03 & 0.21 & 0.01 & 0.00 & 0.00 & 0.01 & 0.02 & 0.02 & 0.01 & 0.00 & 0.00 & 0.00 \\
\hline \multirow{6}{*}{$\sigma$} & $v_{1}$ & 0.01 & 0.01 & 0.01 & 0.04 & 0.16 & 0.02 & 0.00 & 0.00 & 0.01 & 0.02 & 0.03 & 0.01 & 0.00 & 0.00 & 0.01 \\
\hline & $a_{1}$ & 0.02 & 0.01 & 0.00 & 0.00 & 0.04 & 0.02 & 0.01 & 0.00 & 0.00 & 0.04 & 0.02 & 0.02 & 0.00 & 0.00 & 0.14 \\
\hline & $\Delta_{1}$ & 0.01 & 0.01 & 0.01 & 0.03 & 0.21 & 0.01 & 0.00 & 0.00 & 0.00 & 0.02 & 0.02 & 0.04 & 0.00 & 0.01 & 0.15 \\
\hline & $\nabla_{1}^{1}$ & 0.01 & 0.01 & 0.01 & 0.04 & 0.16 & 0.01 & 0.01 & 0.00 & 0.00 & 0.03 & 0.02 & 0.04 & 0.01 & 0.01 & 0.24 \\
\hline & $V_{\mathrm{S} 1}^{1}$ & 0.01 & 0.02 & 0.01 & 0.04 & 0.62 & 0.04 & 0.00 & 0.00 & 0.00 & 0.06 & 0.01 & 0.02 & 0.00 & 0.01 & 0.03 \\
\hline & $V_{\mathrm{D} 1}$ & 0.02 & 0.01 & 0.01 & 0.03 & 0.16 & 0.03 & 0.00 & 0.00 & 0.02 & 0.06 & 0.08 & 0.08 & 0.01 & 0.04 & 0.33 \\
\hline \multirow{8}{*}{$\mu$} & $u_{2}$ & -0.60 & -0.29 & -0.09 & 0.09 & 1.19 & -0.28 & -0.11 & -0.03 & 0.03 & 0.19 & -0.29 & -0.04 & 0.01 & 0.05 & 0.12 \\
\hline & $v_{2}$ & -0.70 & -0.43 & -0.18 & 0.10 & 1.12 & -0.28 & -0.13 & -0.05 & 0.03 & 0.19 & -0.34 & -0.03 & 0.05 & 0.19 & 0.34 \\
\hline & $a_{2}$ & -0.68 & -0.62 & -0.49 & -0.22 & 0.00 & -0.26 & -0.08 & 0.06 & 0.96 & 1.81 & -0.23 & 0.03 & 0.34 & 1.71 & 4.34 \\
\hline & $\Delta_{2}$ & -0.60 & -0.29 & -0.09 & 0.09 & 1.19 & -0.15 & -0.04 & 0.00 & 0.09 & 0.40 & -0.49 & -0.16 & 0.11 & 0.66 & 2.98 \\
\hline & $\nabla_{2}$ & -0.70 & -0.43 & -0.18 & 0.10 & 1.12 & -0.16 & -0.02 & 0.08 & 0.57 & 1.51 & -0.50 & -0.12 & 0.41 & 2.37 & 8.78 \\
\hline & $V_{\mathrm{S} 2}^{2}$ & -0.67 & -0.37 & -0.14 & 0.08 & 3.48 & -0.27 & -0.05 & 0.02 & 0.17 & 0.75 & -0.57 & -0.18 & 0.19 & 1.07 & 5.71 \\
\hline & $V_{\mathrm{D} 2}^{\mathrm{S} 2}$ & -0.68 & -0.41 & -0.17 & 0.10 & 1.09 & -0.16 & -0.01 & 0.08 & 0.56 & 1.50 & -0.46 & -0.07 & 0.38 & 2.33 & 8.71 \\
\hline & $u_{2}$ & 0.01 & 0.01 & 0.01 & 0.01 & 0.14 & 0.01 & 0.00 & 0.00 & 0.00 & 0.01 & 0.03 & 0.01 & 0.00 & 0.00 & 0.00 \\
\hline \multirow{6}{*}{$\sigma$} & $v_{2}$ & 0.01 & 0.01 & 0.01 & 0.01 & 0.13 & 0.02 & 0.00 & 0.00 & 0.00 & 0.01 & 0.03 & 0.00 & 0.01 & 0.00 & 0.01 \\
\hline & $a_{2}$ & 0.01 & 0.00 & 0.00 & 0.01 & 0.02 & 0.02 & 0.01 & 0.02 & 0.11 & 0.17 & 0.04 & 0.02 & 0.04 & 0.19 & 0.38 \\
\hline & $\Delta_{2}$ & 0.01 & 0.01 & 0.01 & 0.01 & 0.14 & 0.02 & 0.01 & 0.01 & 0.02 & 0.04 & 0.02 & 0.03 & 0.02 & 0.07 & 0.19 \\
\hline & $\nabla_{2}$ & 0.01 & 0.01 & 0.01 & 0.01 & 0.13 & 0.02 & 0.01 & 0.02 & 0.09 & 0.14 & 0.03 & 0.02 & 0.07 & 0.24 & 0.70 \\
\hline & $V_{\mathrm{S} 2}^{2}$ & 0.01 & 0.02 & 0.02 & 0.02 & 0.39 & 0.04 & 0.01 & 0.01 & 0.02 & 0.09 & 0.02 & 0.03 & 0.02 & 0.10 & 0.72 \\
\hline & $V_{\mathrm{D} 2}^{\mathrm{L} 2}$ & 0.02 & 0.01 & 0.01 & 0.01 & 0.13 & 0.04 & 0.00 & 0.02 & 0.09 & 0.13 & 0.12 & 0.02 & 0.06 & 0.23 & 0.68 \\
\hline \multirow{7}{*}{$\mu$} & $u_{3}$ & -0.55 & -0.26 & -0.07 & 0.10 & 1.32 & -0.30 & -0.11 & -0.02 & 0.03 & 0.20 & -0.29 & -0.04 & 0.00 & 0.05 & 0.09 \\
\hline & $v_{3}$ & -0.59 & -0.35 & -0.13 & 0.15 & 1.45 & -0.27 & -0.12 & -0.04 & 0.04 & 0.24 & -0.35 & -0.04 & 0.04 & 0.18 & 0.32 \\
\hline & $a_{3}$ & -0.50 & -0.27 & -0.17 & -0.09 & 0.13 & -0.30 & -0.08 & 0.00 & 0.40 & 0.68 & -0.31 & -0.01 & 0.19 & 1.09 & 2.07 \\
\hline & $\Delta_{3}$ & -0.55 & -0.26 & -0.07 & 0.10 & 1.32 & -0.16 & -0.05 & 0.00 & 0.05 & 0.20 & -0.55 & -0.22 & 0.05 & 0.42 & 1.79 \\
\hline & $\nabla_{3}$ & -0.59 & -0.35 & -0.13 & 0.15 & 1.45 & -0.18 & -0.04 & 0.04 & 0.39 & 0.74 & -0.58 & -0.23 & 0.22 & 1.33 & 4.10 \\
\hline & $V_{\mathrm{S} 3}$ & -0.59 & -0.31 & -0.10 & 0.12 & 4.54 & -0.28 & -0.06 & 0.00 & 0.08 & 0.44 & -0.63 & -0.29 & 0.08 & 0.57 & 3.14 \\
\hline & $V_{\mathrm{D} 3}$ & -0.57 & -0.34 & -0.12 & 0.14 & 1.42 & -0.18 & -0.02 & 0.05 & 0.40 & 0.75 & -0.48 & -0.10 & 0.23 & 1.33 & 4.11 \\
\hline
\end{tabular}


Table 2. Cont.

\begin{tabular}{|c|c|c|c|c|c|c|c|c|c|c|c|c|c|c|c|c|}
\hline & & \multicolumn{5}{|c|}{ First Story } & \multicolumn{5}{|c|}{ Average Across Stories } & \multicolumn{5}{|c|}{ Roof } \\
\hline & & $\min$ & $x^{10}$ & $x^{50}$ & $x^{90}$ & $\max$ & $\min$ & $x^{10}$ & $x^{50}$ & $x^{90}$ & $\max$ & $\min$ & $x^{10}$ & $x^{50}$ & $x^{90}$ & $\max$ \\
\hline \multirow{7}{*}{$\sigma$} & $u_{3}$ & 0.02 & 0.01 & 0.01 & 0.01 & 0.16 & 0.02 & 0.00 & 0.00 & 0.00 & 0.02 & 0.03 & 0.01 & 0.00 & 0.01 & 0.00 \\
\hline & $v_{3}$ & 0.02 & 0.01 & 0.01 & 0.02 & 0.14 & 0.01 & 0.00 & 0.00 & 0.00 & 0.01 & 0.03 & 0.00 & 0.01 & 0.01 & 0.01 \\
\hline & $a_{3}$ & 0.03 & 0.01 & 0.01 & 0.01 & 0.04 & 0.02 & 0.01 & 0.01 & 0.07 & 0.09 & 0.04 & 0.02 & 0.05 & 0.15 & 0.38 \\
\hline & $\Delta_{3}$ & 0.02 & 0.01 & 0.01 & 0.01 & 0.16 & 0.01 & 0.01 & 0.00 & 0.02 & 0.02 & 0.03 & 0.04 & 0.01 & 0.06 & 0.15 \\
\hline & $\nabla_{3}$ & 0.02 & 0.01 & 0.01 & 0.02 & 0.14 & 0.01 & 0.01 & 0.02 & 0.04 & 0.05 & 0.02 & 0.04 & 0.04 & 0.08 & 0.27 \\
\hline & $V_{\mathrm{S} 3}^{3}$ & 0.01 & 0.02 & 0.02 & 0.02 & 0.41 & 0.04 & 0.01 & 0.00 & 0.02 & 0.07 & 0.03 & 0.03 & 0.01 & 0.07 & 0.27 \\
\hline & $V_{\mathrm{D} 3}^{50}$ & 0.02 & 0.01 & 0.01 & 0.02 & 0.13 & 0.03 & 0.00 & 0.02 & 0.04 & 0.05 & 0.10 & 0.03 & 0.04 & 0.08 & 0.26 \\
\hline
\end{tabular}

Table 3. Shear-type cases (B0- $\infty$, B3- $\infty$, B5- $\infty, C 0-\infty, C 3-\infty, C 5-\infty)$, mean $\mu$ and standard deviation $\sigma$ of error across cases.

\begin{tabular}{|c|c|c|c|c|c|c|c|c|c|c|c|c|c|c|c|c|}
\hline & & \multicolumn{5}{|c|}{ First Story } & \multicolumn{5}{|c|}{ Average Across Stories } & \multicolumn{5}{|c|}{ Roof } \\
\hline & & $\min$ & $x^{10}$ & $x^{50}$ & $x^{90}$ & $\max$ & $\min$ & $x^{10}$ & $x^{50}$ & $x^{90}$ & $\max$ & $\min$ & $x^{10}$ & $x^{50}$ & $x^{90}$ & $\max$ \\
\hline \multirow{7}{*}{$\mu$} & $u_{1}$ & -0.40 & -0.10 & 0.03 & 0.36 & 2.95 & -0.30 & -0.06 & 0.01 & 0.07 & 0.44 & -0.46 & -0.13 & -0.01 & 0.01 & 0.06 \\
\hline & $v_{1}$ & -0.41 & -0.12 & 0.05 & 0.48 & 2.98 & -0.32 & -0.06 & 0.01 & 0.10 & 0.45 & -0.46 & -0.13 & -0.01 & 0.02 & 0.11 \\
\hline & $a_{1}$ & -0.49 & -0.16 & -0.01 & 0.04 & 0.49 & -0.46 & -0.18 & -0.02 & 0.03 & 0.55 & -0.63 & -0.34 & -0.02 & 0.07 & 1.70 \\
\hline & $\Delta_{1}$ & -0.40 & -0.10 & 0.03 & 0.36 & 2.95 & -0.30 & -0.11 & -0.01 & 0.02 & 0.26 & -0.79 & -0.47 & -0.03 & 0.25 & 2.83 \\
\hline & $\nabla_{1}$ & -0.41 & -0.12 & 0.05 & 0.48 & 2.98 & -0.30 & -0.12 & -0.02 & 0.03 & 0.42 & -0.85 & -0.59 & -0.05 & 0.33 & 3.76 \\
\hline & $V_{\mathrm{S} 1}^{1}$ & -0.40 & -0.10 & 0.03 & 0.36 & 2.95 & -0.30 & -0.11 & -0.01 & 0.02 & 0.26 & -0.79 & -0.47 & -0.03 & 0.25 & 2.83 \\
\hline & $V_{\mathrm{D} 1}^{\mathrm{S1}}$ & -0.40 & -0.11 & 0.05 & 0.46 & 2.92 & -0.26 & -0.08 & -0.01 & 0.05 & 0.47 & -0.66 & -0.30 & -0.02 & 0.36 & 3.72 \\
\hline \multirow{8}{*}{$\sigma$} & $u_{1}$ & 0.00 & 0.00 & 0.00 & 0.02 & 0.07 & 0.01 & 0.00 & 0.00 & 0.01 & 0.02 & 0.05 & 0.01 & 0.00 & 0.00 & 0.00 \\
\hline & $v_{1}$ & 0.01 & 0.00 & 0.00 & 0.01 & 0.16 & 0.01 & 0.00 & 0.00 & 0.01 & 0.03 & 0.04 & 0.01 & 0.00 & 0.00 & 0.00 \\
\hline & $a_{1}$ & 0.02 & 0.00 & 0.00 & 0.00 & 0.02 & 0.03 & 0.01 & 0.00 & 0.00 & 0.06 & 0.03 & 0.02 & 0.00 & 0.01 & 0.23 \\
\hline & $\Delta_{1}$ & 0.00 & 0.00 & 0.00 & 0.02 & 0.07 & 0.03 & 0.01 & 0.00 & 0.00 & 0.01 & 0.01 & 0.01 & 0.00 & 0.00 & 0.03 \\
\hline & $\nabla_{1}^{1}$ & 0.01 & 0.00 & 0.00 & 0.01 & 0.16 & 0.02 & 0.01 & 0.00 & 0.00 & 0.03 & 0.01 & 0.01 & 0.00 & 0.01 & 0.12 \\
\hline & $V_{\mathrm{S} 1}^{1}$ & 0.00 & 0.00 & 0.00 & 0.02 & 0.07 & 0.03 & 0.01 & 0.00 & 0.00 & 0.01 & 0.01 & 0.01 & 0.00 & 0.00 & 0.03 \\
\hline & $V_{\mathrm{D} 1}^{\mathrm{N}}$ & 0.01 & 0.00 & 0.00 & 0.01 & 0.16 & 0.04 & 0.00 & 0.00 & 0.02 & 0.06 & 0.13 & 0.07 & 0.04 & 0.04 & 0.11 \\
\hline & $u_{2}$ & -0.56 & -0.24 & -0.03 & 0.20 & 1.77 & -0.36 & -0.11 & -0.01 & 0.03 & 0.20 & -0.44 & -0.08 & 0.01 & 0.06 & 0.15 \\
\hline \multirow{6}{*}{$\mu$} & $v_{2}$ & -0.65 & -0.35 & -0.10 & 0.22 & 1.70 & -0.37 & -0.12 & -0.02 & 0.03 & 0.21 & -0.44 & -0.06 & 0.04 & 0.19 & 0.37 \\
\hline & $a_{2}$ & -0.73 & -0.61 & -0.50 & -0.26 & 0.12 & -0.34 & -0.12 & 0.02 & 0.67 & 1.64 & -0.32 & -0.02 & 0.29 & 1.51 & 4.32 \\
\hline & $\Delta_{2}$ & -0.56 & -0.24 & -0.03 & 0.20 & 1.77 & -0.29 & -0.09 & 0.01 & 0.13 & 0.52 & -0.58 & -0.31 & 0.13 & 0.81 & 4.34 \\
\hline & $\nabla_{2}$ & -0.65 & -0.35 & -0.10 & 0.22 & 1.70 & -0.29 & -0.06 & 0.09 & 0.63 & 1.61 & -0.59 & -0.27 & 0.41 & 2.50 & 10.50 \\
\hline & $V_{\mathrm{S} 2}^{2}$ & -0.56 & -0.24 & -0.03 & 0.20 & 1.77 & -0.29 & -0.09 & 0.01 & 0.13 & 0.52 & -0.58 & -0.31 & 0.13 & 0.81 & 4.34 \\
\hline & $V_{\mathrm{D} 2}^{\mathrm{S} 2}$ & -0.64 & -0.34 & -0.10 & 0.21 & 1.65 & -0.26 & -0.03 & 0.09 & 0.62 & 1.59 & -0.57 & -0.10 & 0.39 & 2.44 & 10.35 \\
\hline \multirow{7}{*}{$\sigma$} & $u_{2}$ & 0.01 & 0.01 & 0.01 & 0.00 & 0.02 & 0.01 & 0.00 & 0.00 & 0.00 & 0.02 & 0.05 & 0.01 & 0.00 & 0.00 & 0.01 \\
\hline & $v_{2}$ & 0.00 & 0.00 & 0.01 & 0.00 & 0.05 & 0.01 & 0.00 & 0.00 & 0.00 & 0.01 & 0.04 & 0.01 & 0.01 & 0.00 & 0.01 \\
\hline & $a_{2}$ & 0.00 & 0.00 & 0.01 & 0.01 & 0.02 & 0.03 & 0.01 & 0.02 & 0.12 & 0.23 & 0.06 & 0.04 & 0.06 & 0.25 & 0.55 \\
\hline & $\Delta_{2}$ & 0.01 & 0.01 & 0.01 & 0.00 & 0.02 & 0.03 & 0.01 & 0.01 & 0.02 & 0.03 & 0.04 & 0.03 & 0.03 & 0.07 & 0.17 \\
\hline & $\nabla_{2}$ & 0.00 & 0.00 & 0.01 & 0.00 & 0.05 & 0.02 & 0.01 & 0.03 & 0.10 & 0.19 & 0.02 & 0.03 & 0.08 & 0.29 & 1.08 \\
\hline & $V_{\mathrm{S} 2}^{2}$ & 0.01 & 0.01 & 0.01 & 0.00 & 0.02 & 0.03 & 0.01 & 0.01 & 0.02 & 0.03 & 0.04 & 0.03 & 0.03 & 0.07 & 0.17 \\
\hline & $V_{\mathrm{D} 2}$ & 0.01 & 0.01 & 0.00 & 0.01 & 0.07 & 0.06 & 0.01 & 0.02 & 0.10 & 0.18 & 0.16 & 0.04 & 0.06 & 0.28 & 1.03 \\
\hline & $u_{3}$ & -0.52 & -0.19 & -0.01 & 0.24 & 2.10 & -0.36 & -0.10 & 0.00 & 0.03 & 0.26 & -0.45 & -0.09 & 0.00 & 0.04 & 0.11 \\
\hline \multirow{6}{*}{$\mu$} & $v_{3}$ & -0.55 & -0.28 & -0.05 & 0.28 & 2.15 & -0.37 & -0.11 & -0.02 & 0.04 & 0.26 & -0.45 & -0.07 & 0.03 & 0.17 & 0.34 \\
\hline & $a_{3}$ & -0.63 & -0.32 & -0.17 & -0.08 & 0.33 & -0.36 & -0.14 & -0.03 & 0.22 & 0.66 & -0.42 & -0.09 & 0.12 & 0.85 & 2.21 \\
\hline & $\Delta_{3}$ & -0.52 & -0.19 & -0.01 & 0.24 & 2.10 & -0.30 & -0.10 & 0.00 & 0.06 & 0.32 & -0.68 & -0.39 & 0.03 & 0.47 & 3.19 \\
\hline & $\nabla_{3}$ & -0.55 & -0.28 & -0.05 & 0.28 & 2.15 & -0.30 & -0.08 & 0.04 & 0.39 & 0.84 & -0.69 & -0.42 & 0.19 & 1.32 & 5.48 \\
\hline & $V_{\mathrm{S} 3}$ & -0.52 & -0.19 & -0.01 & 0.24 & 2.10 & -0.30 & -0.10 & 0.00 & 0.06 & 0.32 & -0.68 & -0.39 & 0.03 & 0.47 & 3.19 \\
\hline & $V_{\mathrm{D} 3}^{\mathrm{s} 3}$ & -0.54 & -0.27 & -0.05 & 0.27 & 2.10 & -0.26 & -0.04 & 0.05 & 0.40 & 0.84 & -0.60 & -0.15 & 0.20 & 1.31 & 5.45 \\
\hline \multirow{7}{*}{$\sigma$} & $u_{3}$ & 0.03 & 0.01 & 0.01 & 0.01 & 0.18 & 0.01 & 0.00 & 0.00 & 0.00 & 0.04 & 0.05 & 0.01 & 0.00 & 0.01 & 0.01 \\
\hline & $v_{3}$ & 0.02 & 0.00 & 0.01 & 0.00 & 0.14 & 0.01 & 0.00 & 0.00 & 0.00 & 0.01 & 0.04 & 0.01 & 0.01 & 0.01 & 0.02 \\
\hline & $a_{3}$ & 0.02 & 0.00 & 0.02 & 0.02 & 0.06 & 0.04 & 0.02 & 0.01 & 0.06 & 0.11 & 0.05 & 0.04 & 0.04 & 0.20 & 0.53 \\
\hline & $\Delta_{3}$ & 0.03 & 0.01 & 0.01 & 0.01 & 0.18 & 0.03 & 0.01 & 0.00 & 0.02 & 0.01 & 0.03 & 0.02 & 0.01 & 0.06 & 0.07 \\
\hline & $\nabla_{3}$ & 0.02 & 0.00 & 0.01 & 0.00 & 0.14 & 0.02 & 0.01 & 0.02 & 0.07 & 0.09 & 0.02 & 0.02 & 0.05 & 0.15 & 0.24 \\
\hline & $V_{\mathrm{S} 3}^{3}$ & 0.03 & 0.01 & 0.01 & 0.01 & 0.18 & 0.03 & 0.01 & 0.00 & 0.02 & 0.01 & 0.03 & 0.02 & 0.01 & 0.06 & 0.07 \\
\hline & $V_{\mathrm{D} 3}$ & 0.02 & 0.00 & 0.00 & 0.01 & 0.15 & 0.04 & 0.01 & 0.02 & 0.07 & 0.10 & 0.16 & 0.04 & 0.04 & 0.15 & 0.25 \\
\hline
\end{tabular}

\section{Discussion}

\subsection{Error Results for THMA (Error 1)}

The following observations are derived from the information in plots and tables:

- As shown in Figures 3 and A3, Figures A4 and A5, Tables 2 and 3, the median $x^{50}$ for all cases, variables and stories is very close to 0 , and the percentiles $x^{10}$ and $x^{90}$ are approximately contained within the indicative EAR $[-0.15 ;+0.25]$ except at roof, where a tendency for under-prediction $\left(x^{10}\right.$ below -0.15$)$ exists for all variables except $u_{1, \text { error,roof }}$ for this variable the prediction is excellent, as expected from the fact that 
the roof displacement is the key variable in the modal method. Indeed, the relative velocity error $v_{1 \text {,error,roof }}$ is also very small. However, this is not the case for $a_{1 \text {,error,roof }}$.

- In general, the dispersion for all variables (except $u_{1}$ and $v_{1}$ ) is smaller in central stories of the frame and increases at the top and bottom (Figure 3); the 3-story frame is an exception where $x^{90}$ and max generally diminish at the top.

- The dispersion $\sigma$ across cases included in Tables 2 and 3 is very small for all statistics except max, indicating a similar behavior across cases.

- When the average across stories is examined (Figures A3-A5, Tables 2 and 3), THMA offers a reasonable accuracy for all variables analyzed. The behavior is particularly

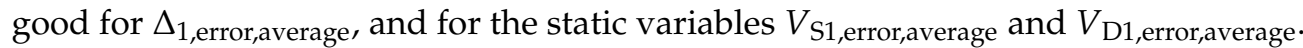

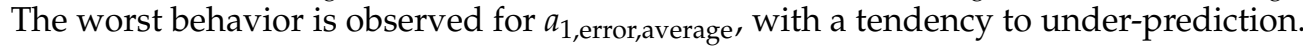
In all cases, a reduced number of extreme values give errors well outside the indicative EAR. Extreme errors are more pronounced for shear-type systems except for $V_{\mathrm{S} 1 \text {,error,average }}$

- When the first story error is examined, larger extreme errors are found, particularly for the maximum in all variables except $a_{1 \text {,error,first }}$. The over-conservative error reaches particularly high values (up to 6.66 for B5-1) for $V_{\mathrm{S} 1 \text {,error,first }}$. For other variables, approximately up to 3 . Remarkable minimum extreme errors are also found.

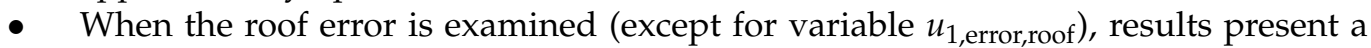
larger dispersion, and in general are more un-conservative than those of the average or first story. The minimum errors are more extreme for this story, and in fact $x^{10}$ for most cases generally falls below -0.15 ; large maximum errors are also found, in some variables ( $\left.a_{1 \text {,error,roof }}, V_{\mathrm{D} 1 \text {,error,roof }}\right)$ even above the corresponding maxima of the first story error.

- Regarding the influence of inherent damping, comparison of results between B0-1, B3-1 and B5-1 in Figures A3-A5, are virtually identical for all variables and stories, showing that this parameter does not influence the error; because inherent damping has been modeled as modal damping this result was expected.

- $\quad$ Regarding the influence of ground type, comparison of error results between B3-1 vs. C3-1, and B3- $\infty$ vs. C3- $\infty$ show that this parameter exerts a very mild influence on the error. The influence is only apparent in the extreme errors, particularly in the maximum error, but $x^{10}, x^{50}$ and $x^{90}$ are virtually unaffected. This influence is due to the mean spectrum peculiarities of each set.

- $\quad$ Shear-type and frame-type systems render similar median errors, but with a larger dispersion in the former. As is clear in Figures A3-A5, the median value $x^{50}$ is quite stable across cases, but the min and $x^{10}$ decrease for shear-type systems, whereas the max and $x^{90}$ increase. These differences are also clear in Figure 3. Therefore, the maximum and minimum errors expected are those corresponding to shear-type

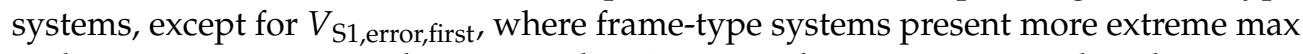
and min errors. It is worth noticing that $\Delta_{1 \text {,error }}$ and $V_{\mathrm{S} 1 \text {,error }}$ are equal in shear-type systems, whereas they differ in frame-type systems.

- It is concluded that although average errors across the system height tend to be centered around 0 , individual stories (particularly first story and roof) will present large errors; the tendency of average errors to be small is due to the negative correlation between errors at the first story and roof; this trend is clear in Figure 6, where a scatter plot of first story THMA error (error 1) versus THMA roof error for all variables is presented; the plot shows that positive errors at roof are generally accompanied by negative errors at first story, and conversely; due to the different sign of top and bottom error, the average across stories is small. This stands for all variables except displacement $u_{1}$ (due to the small error at roof) and acceleration $a_{1}$.

\subsection{Error Results for RSA-SRSS (Error 2) and RSA-CQC (Error 3)}

The following remarks apply to RSA-SRSS and RSA-CQC and are based on numerical data in Tables 2 and 3 (plots are provided as Supplementary Materials, Figures S1-S9): 
- The general trends are very similar between both combination rules and THMA, except for a larger dispersion in most of the variables, and substantial differences in $a$.

- Considering the average across stories, the median $x^{50}$ is close to 0 and the dispersion (measured by $x^{10}$ and $x^{90}$ ) is moderate, except for $\nabla, a$, and $\mathrm{V}_{\mathrm{S}}$. The over-conservative error (max) is considerably smaller for all variables (except $u$ and $v$ ) when the RSACQC method (error 3) is used.

- Considering the first story error, the median $x^{50}$ is negative with both methods and for all variables, indicating that both RSA-SRSS and RSA-CQC tend to under-predict results at this story. However, RSA-SRSS (error 2) renders slightly more un-conservative results, therefore more extreme min values. However, larger over-conservative results for $x^{90}$ and max are obtained with RSA-CQC (error 3).

- Considering the roof error, both methods tend to conservative $(>0)$ median $x^{50}$ results. More extreme un-conservative results min and $x^{10}$ are obtained with RSA-CQC (error 3), but more extreme conservative results $x^{90}$ and max are obtained with RSASRSS (error 2).

- The influence of inherent damping, type of system (shear-type or frame-type) and ground type follows the same trends as in THMA.

- As a conclusion, RSA-SRSS and RSA-CQC methods are expected to render reasonable average results across stories but generally produce un-conservative results for first story and over-conservative results for the roof. Dispersion (measured as $x^{90}-x^{10}$ ) is larger for RSA-SRSS. Overall, both methods are very similar for $u$ and $v$. RSA-CQC offers better results for all other variables.

\subsection{Identification of Significant Parameters}

The error results presented indicate that either method can render un-conservative or over-conservative results for certain cases (combinations of frame and added damping system). It is of interest to link the error to specific values of parameters describing the system. Such identification could be used to impose additional conditions to damped systems analyzed with classical modal procedures to keep the error below a certain threshold. In order to identify the relevant parameters, the variability of the THMA error (error 1) statistics $\min , x^{10}, x^{90}$ and max with respect to the parameters considered in the study is analyzed through their coefficient of variation $c_{v}$ calculated as follows: for a certain case the error results are sorted according to a certain parameter, thus obtaining 4 independent subsets, each one associated with a specific value of the parameter; statistics ( $\min , x^{10}$, $x^{90}$ and max) of each sub-set are calculated; the sample standard deviation $\sigma$ and mean $\mu$ across the four values for each statistic are derived and $c_{v}$ is found as $\sigma / \mu$. An example for case B0- $\infty$ and $\Delta_{1, \text { error }}$ is shown in Table 4 ; in this table, the second column on the left indicates that the largest variation of the min of $\Delta_{1, \text { error,first }}$ is due to $\alpha_{k}(0.63$, highlighted in bold), and the second largest variation is due to $\alpha_{d}(0.36$, highlighted in italics).

Table 4. Coefficient of variation $c_{v}$ for $\Delta_{1, \text { error }}$ and case B0- $\infty$.

\begin{tabular}{|c|c|c|c|c|c|c|c|c|c|c|c|c|c|c|c|c|}
\hline & \multicolumn{4}{|c|}{ First } & \multicolumn{4}{|c|}{ Average } & \multicolumn{4}{|c|}{ Central } & \multicolumn{4}{|c|}{ Roof } \\
\hline & $\min$ & $x^{10}$ & $x^{90}$ & $\max$ & $\min$ & $x^{10}$ & $x^{90}$ & $\max$ & $\min$ & $x^{10}$ & $x^{90}$ & $\max$ & $\min$ & $x^{10}$ & $x^{90}$ & $\max$ \\
\hline$T_{1}$ & 0.09 & 0.22 & 0.44 & 0.37 & 0.08 & 0.07 & 0.47 & 0.31 & 0.07 & 0.13 & 0.59 & 0.38 & 0.11 & 0.25 & 0.25 & 0.25 \\
\hline$\xi_{a, 1}$ & 0.26 & 0.39 & 0.50 & 0.39 & 0.22 & 0.29 & 0.96 & 0.67 & 0.30 & 0.31 & 0.20 & 0.53 & 0.17 & 0.47 & 0.65 & 0.66 \\
\hline$N$ & 0.05 & 0.20 & 0.11 & 0.27 & 0.06 & 0.22 & 0.55 & 0.21 & 0.09 & 0.20 & 0.63 & 0.71 & 0.02 & 0.01 & 0.48 & 0.61 \\
\hline$\alpha_{k}$ & 0.63 & 0.84 & 0.08 & 0.12 & 0.02 & 0.19 & 0.48 & 0.78 & 0.02 & 0.59 & 0.39 & 0.77 & 0.02 & 0.01 & 0.90 & 1.00 \\
\hline$\beta_{k}$ & 0.07 & 0.32 & 0.11 & 0.09 & 0.04 & 0.18 & 0.26 & 0.19 & 0.03 & 0.18 & 0.41 & 0.16 & 0.02 & 0.02 & 0.32 & 0.45 \\
\hline$\alpha_{d}$ & 0.36 & 1.08 & 1.36 & 1.40 & 0.32 & 0.75 & 1.08 & 0.32 & 0.17 & 0.39 & 1.42 & 1.44 & 1.01 & 1.39 & 1.17 & 0.77 \\
\hline$\beta_{d}$ & 0.00 & 0.08 & 0.83 & 0.82 & 0.13 & 0.49 & 0.10 & 0.00 & 0.11 & 0.56 & 0.89 & 0.20 & 0.05 & 0.18 & 0.13 & 0.00 \\
\hline
\end{tabular}

Maximum and second maximum $c_{v}$ for each column identified in bold and italics, respectively. 
This procedure is repeated for each shear-type case (B0- $\infty$, B3- $\infty, \mathrm{B} 5-\infty, \mathrm{C} 0-\infty, \mathrm{C} 3-\infty$, C5- $\infty$ ); the most and second most influential parameter for each case are identified, and the

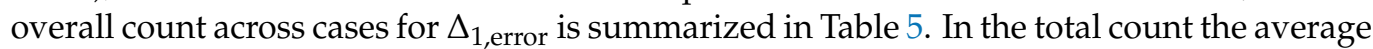
has been left out to avoid double-counting. This table shows a remarkable consistency of the error dependency from certain parameters between cases, but the relevant parameters are not the same across stories and variable statistics. For instance, the min error at first story $\left(\Delta_{1, \text { min,first }}\right)$ is mostly dependent on $\alpha_{d}$ and $\alpha_{k}$; however, in all other stories $\left(\Delta_{1, \text { min,average, }}\right.$ $\left.\Delta_{1, \text { min,central }}, \Delta_{1, \text { min,roof }}\right)$ it is dependent on $\alpha_{d}$ and $\xi_{a, 1}$. The global count indicates that, overall and for extreme errors (max and $\mathrm{min}$ ), the most relevant parameter is $\alpha_{d}$, followed by $\alpha_{k}$. Very similar tables (not shown for brevity) are obtained for $u_{1, \text { error }}, v_{1, \text { error }}, \Delta_{1 \text {,error }}$, $\nabla_{1 \text {,error }}, V_{\mathrm{S} 1 \text {,error }}$ and $V_{\mathrm{D} 1 \text {,error }}$. However, for $a_{1 \text {,error }}$, the most relevant parameter is $T_{1}$ followed by $\alpha_{d}$.

Table 5. Relevance of parameters for $\Delta_{1 \text {,error }}$ and shear-type frames (B0- $\infty$, B3- $\infty$, B5- $\left.\infty, \mathrm{C} 0-\infty, \mathrm{C} 3-\infty, \mathrm{C} 5-\infty\right)$.

\begin{tabular}{|c|c|c|c|c|c|c|c|c|c|c|c|c|c|c|c|c|c|c|c|c|c|}
\hline & \multicolumn{4}{|c|}{ First } & \multicolumn{4}{|c|}{ Average } & \multicolumn{4}{|c|}{ Central } & \multicolumn{4}{|c|}{ Roof } & \multicolumn{5}{|c|}{ Count (Excluding Average) } \\
\hline & $\min$ & $x^{10}$ & $x^{90}$ & $\max$ & $\min$ & $x^{10}$ & $x^{90}$ & $\max$ & $\min$ & $x^{10}$ & $x^{90}$ & $\max$ & $\min$ & $x^{10}$ & $x^{90}$ & $\max$ & $\min$ & $x^{10}$ & $x^{90}$ & $\max$ & All \\
\hline$T_{1}$ & 0 & 0 & 0 & 0 & 0 & 0 & 0 & 0 & 0 & 0 & 0 & 0 & 0 & 0 & 0 & 0 & 0 & 0 & 0 & 0 & 0 \\
\hline$\xi_{a, 1}$ & 0 & 0 & 0 & 0 & 6 & 0 & 6 & 6 & 6 & 0 & 0 & 0 & 6 & 6 & 0 & 0 & 12 & 6 & 0 & 0 & 18 \\
\hline$N$ & 0 & 0 & 0 & 0 & 0 & 0 & 0 & 0 & 0 & 0 & 2 & 4 & 0 & 0 & 0 & 0 & 0 & 0 & 2 & 4 & 6 \\
\hline$\alpha_{k}$ & 6 & 6 & 0 & 0 & 0 & 0 & 0 & 6 & 0 & 6 & 0 & 2 & 0 & 0 & 6 & 6 & 6 & 12 & 6 & 8 & 32 \\
\hline$\beta_{k}^{\wedge}$ & 0 & 0 & 0 & 0 & 0 & 0 & 0 & 0 & 0 & 0 & 0 & 0 & 0 & 0 & 0 & 0 & 0 & 0 & 0 & 0 & 0 \\
\hline$\alpha_{d}$ & 6 & 6 & 6 & 6 & 6 & 6 & 6 & 0 & 6 & 0 & 6 & 6 & 6 & 6 & 6 & 6 & 18 & 12 & 18 & 18 & 66 \\
\hline$\beta_{d}$ & 0 & 0 & 6 & 6 & 0 & 6 & 0 & 0 & 0 & 6 & 4 & 0 & 0 & 0 & 0 & 0 & 0 & 6 & 10 & 6 & 22 \\
\hline
\end{tabular}

The information in Table 4 is used to produce Table 6 in the following way: first, the error results for the case considered are sorted according to the variables with the highest and second highest $c_{v}$ listed in Table 4, thus obtaining 16 independent data subsets, each one associated with a specific value of the first and second parameters. Then the corresponding statistic ( $\min , x^{10}, x^{90}$ and max) for each subset is found. For instance, the top left sub-table in Table 6 refers to the min of $\Delta_{1 \text {,error,first; }}$ for this variable and story, the most influential parameters (as discussed) are $\alpha_{k}$ and $\alpha_{d}$; the four different values for $\alpha_{k}$ $(0.20,0.40,0.60,0.80)$ are listed as row labels (left), and the four different values for $\alpha_{d}$ $(0,1 / 3,2 / 3,1)$ are listed as column headers (above). The min of $\Delta_{1, \text { error,first for all systems }}$ with $\alpha_{k}=0.40$ and $\alpha_{d}=1 / 3$ can be read in the table as -0.15 . The procedure has been repeated for all shear-type cases; average values between cases obtained are within $\pm 10 \%$ variation of those in Table 6. Thus, given a system with known properties, the error bounds for classical modal analysis can be estimated. For instance, the un-conservative error $(\mathrm{min})$ at roof can be kept almost negligible by choosing a constant damper distribution $\left(\alpha_{d}=1\right)$, regardless of the amount of added damping $\xi_{\mathrm{a} 1}$. However, if $\alpha_{\mathrm{d}}=1 / 3$, the maximum acceptable inherent damping for a -0.15 error threshold would be $10 \%$ (resulting in a min $\left.\Delta_{1 \text {,error,roof }}=-0.14\right)$. It is worth noticing, however, that values of parameters beneficial for some specific target may be simultaneously detrimental for other. For example, the adoption of $\alpha_{d}=1$, while ensuring that the min $\Delta_{1 \text {,error,roof }}$ is negligible (above -0.01 ), is linked to considerable un-conservative errors for the first story ( $\min \Delta_{1, \text { error,first up to }-0.41)}$ and over-conservative errors for the roof $\left(\max \Delta_{1, \text { error,roof }}\right.$ up to +2.78 ). This proves the difficulty of proposing simple rules involving the parameters considered, to keep the error under control. Similar conclusions are obtained for RSA-SRSS and RSA-CQC errors.

\subsection{Correlation of Error Results with Existing Non-Proportionality Indices}

To investigate the relationship between the NPIs described in Section 2.2 and Appendix A with the error results described in Section 3, values of the indices are calculated for each system in the study; for mode-dependent indices, the average value across modes is taken. Index $I_{P F 1}$ is left out because of its numerical instability. Indices $I_{A 1}$ and $I_{L K Z 4}$ are very similar, showing a sample Pearson correlation coefficient $\rho\left(I_{L K Z 4} ; I_{A 1}\right)=0.98$ (calculated including all systems analyzed in this study), for this reason the discussion and conclusions 
for $I_{A 1}$ can be extended to $I_{L K Z 4}$. Two conditions indicate a good correlation of error and index: 1) the dispersion of errors (measured by the difference between extremes) must increase steadily with increasing index value; 2 ) for index values close to 0 the error should be close to 0; limiting the index value will then result in an effective error limitation.

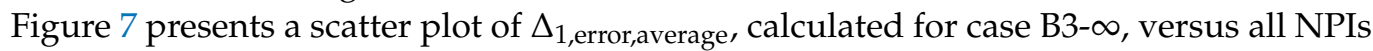
considered in the study (except $I_{P F 1}$ ); each sub-plot contains 13,312 points, each point corresponding to a different system. The plot shows clearly that only four indices $\left(I_{P S 5}\right.$, $I_{T L L 1}, I_{L K Z 4}$ and $\left.I_{A 1}\right)$ fulfil both conditions. Plots for other cases, variables and stories, not shown for brevity, confirm this observation.

Table 6. Min, $x^{10}, x^{90}$ and max for $\Delta_{1 \text {,error }}$ and case B0- $\infty$ sorted by relevant parameters.

\begin{tabular}{|c|c|c|c|c|c|c|c|c|c|c|c|c|c|c|c|c|c|c|c|c|}
\hline & \multicolumn{5}{|c|}{ First Story } & \multicolumn{5}{|c|}{ Average Across Stories } & \multicolumn{5}{|c|}{ Central Story } & \multicolumn{5}{|c|}{ Roof } \\
\hline & $\alpha_{k}$ & 0 & $1 / 3^{\alpha_{d}}$ & $2 / 3$ & 1 & $\alpha_{d}$ & $10^{\circ}$ & $20 \%{ }^{\xi_{a 1}}$ & 3 & $40 \%$ & $\xi_{a 1}$ & 0 & ${ }_{1 / 3}{ }^{\alpha_{d}}$ & $2 / 3$ & 1 & $\alpha_{d}$ & & $20 \%$ & 36 & 40 \\
\hline \multirow{4}{*}{$\min$} & 0.20 & -0.16 & -0.29 & -0.36 & -0.41 & 0 & -0.18 & -0.24 & -0.28 & -0.31 & $10 \%$ & -0.22 & -0.13 & -0.14 & -0.17 & 0 & -0.53 & -0.67 & -0.75 & -0.79 \\
\hline & 0.40 & -0.06 & -0.15 & -0.22 & -0.27 & $1 / 3$ & -0.09 & -0.11 & -0.12 & -0.14 & $20 \%$ & -0.36 & -0.23 & -0.22 & -0.25 & $1 / 3$ & -0.14 & -0.24 & -0.34 & -0.41 \\
\hline & 0.60 & -0.01 & -0.05 & -0.11 & -0.16 & $2 / 3$ & -0.11 & -0.14 & -0.18 & -0.20 & $30 \%$ & -0.43 & -0.30 & -0.29 & -0.33 & $2 / 3$ & -0.04 & -0.08 & -0.12 & -0.15 \\
\hline & 0.80 & 0.00 & 0.00 & -0.02 & -0.07 & 1 & -0.13 & -0.17 & -0.22 & -0.24 & $40 \%$ & -0.48 & -0.34 & -0.34 & -0.39 & 1 & -0.01 & -0.01 & 0.00 & 0.03 \\
\hline \multirow{5}{*}{$x^{10}$} & $\alpha_{d}$ & 0.20 & 0.40 & 0.60 & 0.80 & $\alpha_{d}$ & 0. & $0.50^{r}$ & 1.00 & 2 & $\alpha_{k}$ & & 0.50 & 1. & 2. & $\alpha_{d}$ & & $20 \%$ & 30 & \\
\hline & 0 & 0.01 & 0.03 & 0.03 & 0.04 & 0 & -0.25 & -0.14 & -0.12 & -0.10 & 0.20 & -0.28 & -0.21 & -0.21 & -0.21 & 0 & -0.30 & -0.50 & -0.62 & -0.71 \\
\hline & $1 / 3$ & -0.13 & -0.03 & 0.01 & 0.02 & $1 / 3$ & -0.07 & -0.06 & -0.05 & -0.05 & 0.40 & -0.26 & -0.09 & -0.07 & -0.07 & $1 / 3$ & -0.07 & -0.15 & -0.21 & -0.27 \\
\hline & $2 / 3$ & -0.25 & -0.11 & -0.04 & 0.00 & $2 / 3$ & -0.05 & -0.05 & -0.05 & -0.05 & 0.60 & -0.28 & -0.07 & -0.03 & -0.02 & $2 / 3$ & -0.01 & -0.03 & -0.05 & -0.06 \\
\hline & 1 & -0.32 & -0.20 & -0.11 & -0.05 & 1 & -0.06 & -0.06 & -0.06 & -0.06 & 0.80 & -0.31 & -0.09 & -0.02 & -0.01 & 1 & 0.01 & 0.03 & 0.06 & 0.08 \\
\hline \multirow{5}{*}{$x^{90}$} & $\alpha_{d}$ & 0.10 & 0.50 & 1.00 & 2.00 & $\alpha_{d}$ & 1 & $20 \%^{\tilde{\zeta}}$ & & 4 & $\alpha_{d}$ & 0.10 & $0.50^{\beta}$ & 1.00 & 2 & $\alpha_{d}$ & 0 & 0.40 & 0.60 & 0.80 \\
\hline & 0 & 1.53 & 0.60 & 0.34 & 0.18 & 0 & 0.02 & 0.00 & -0.02 & -0.03 & 0 & 0.01 & 0.09 & 0.11 & 0.12 & 0 & -0.10 & -0.10 & -0.11 & -0.12 \\
\hline & $1 / 3$ & 0.40 & 0.21 & 0.13 & 0.06 & $1 / 3$ & 0.00 & 0.00 & 0.00 & 0.01 & $1 / 3$ & 0.01 & 0.02 & 0.03 & 0.0 & $1 / 3$ & 0.44 & 0.06 & -0.03 & -0.04 \\
\hline & $2 / 3$ & 0.10 & 0.06 & 0.03 & 0.01 & $2 / 3$ & 0.00 & 0.01 & 0.03 & 0.06 & $2 / 3$ & -0.01 & 0.00 & 0.01 & 0.0 & $2 / 3$ & 0.90 & 0.36 & 0.11 & 0.00 \\
\hline & 1 & -0.01 & -0.01 & -0.01 & -0.01 & 1 & 0.00 & 0.02 & 0.05 & 0.09 & 1 & 0.00 & 0.00 & 0.00 & 0.00 & 1 & 1.21 & 0.59 & 0.30 & 0.12 \\
\hline \multirow{5}{*}{$\max$} & $\alpha_{d}$ & 0.10 & 0.50 & 1.00 & 2.00 & $\alpha_{k}$ & $10 \%$ & $20 \%$ & $30 \%$ & $40^{\circ}$ & $\alpha_{d}$ & 0.20 & 0.40 & 0.60 & 0.80 & $\alpha_{k}$ & 0 & $1 / 3$ & $2 / 3$ & 1 \\
\hline & 0 & 3.00 & 0.97 & 0.81 & 0.64 & 0.20 & 0.06 & 0.06 & 0.15 & 0.25 & 0 & 0.61 & 0.32 & 0.16 & 0.10 & 0.20 & -0.04 & 1.38 & 2.32 & 2.78 \\
\hline & $1 / 3$ & 0.71 & 0.36 & 0.21 & 0.14 & 0.40 & 0.04 & 0.04 & 0.09 & 0.13 & $1 / 3$ & 0.04 & 0.09 & 0.08 & 0.08 & 0.40 & -0.03 & 0.40 & 0.90 & 1.16 \\
\hline & $2 / 3$ & 0.23 & 0.13 & 0.08 & 0.04 & 0.60 & 0.02 & 0.03 & 0.05 & 0.07 & $2 / 3$ & -0.02 & 0.04 & 0.04 & 0.03 & 0.60 & -0.04 & 0.00 & 0.32 & 0.50 \\
\hline & 1 & 0.00 & 0.00 & 0.00 & 0.00 & 0.80 & 0.01 & 0.01 & 0.02 & 0.03 & 1 & -0.03 & 0.02 & 0.03 & 0.02 & 0.80 & -0.04 & -0.03 & 0.06 & 0.18 \\
\hline
\end{tabular}

The scatter plot for each index, variable, story, and case is enveloped by a minimum monotonically increasing curve and a maximum monotonically decreasing curve, hereinafter referred to as max and min envelopes, respectively. Plot of these envelopes comprising all cases considered in the study are provided in Figure 8 (index $I_{P S 5}$ ), Figure 9 (index $I_{T L L 1}$ ), and Figure 10 (index $I_{A 1}$ ). $I_{L K Z 4}$ is omitted because the corresponding plots are virtually identical to those for $I_{A 1}$. These plots establish a direct relationship between a maximum or minimum value of error for any variable and the relevant NPI at every case. The following conclusions are derived:

- The relationship between THMA error for a certain variable at a certain story and the same NPI follows similar trends for all cases in the study, regardless of the index considered. The curves corresponding to different cases are, in all cases, closely squeezed together.

- The differences between cases are mostly due to model type: shear-type cases (B0- $\infty$, $\mathrm{B} 3-\infty, \mathrm{B} 5-\infty, \mathrm{C} 0-\infty, \mathrm{C} 3-\infty, \mathrm{C} 5-\infty)$ present more extreme errors and generally correspond to the outermost curve; contrariwise, the extreme case of frame-type system (B3-0.10) presents the innermost curve of the collection. The only exception is $V_{\mathrm{S} 1 \text {,average }}$ where for all indices the min un-conservative error corresponds to B3-0.10.

- Inherent damping and ground type exert a very mild influence; for the same $\lambda$, the curves corresponding to different inherent damping ratios are practically identical, as are the curves corresponding to different ground types. An exception happens at

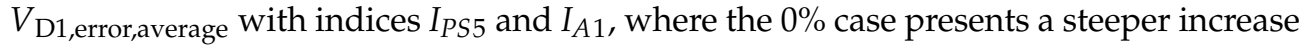
of conservative error. This trend is not detected in index $I_{T L L 1}$.

- The absolute value of un-conservative error is, in general, much smaller than the conservative error. However, for some specific variables and stories, similar orders of magnitude are obtained for both extreme errors. 

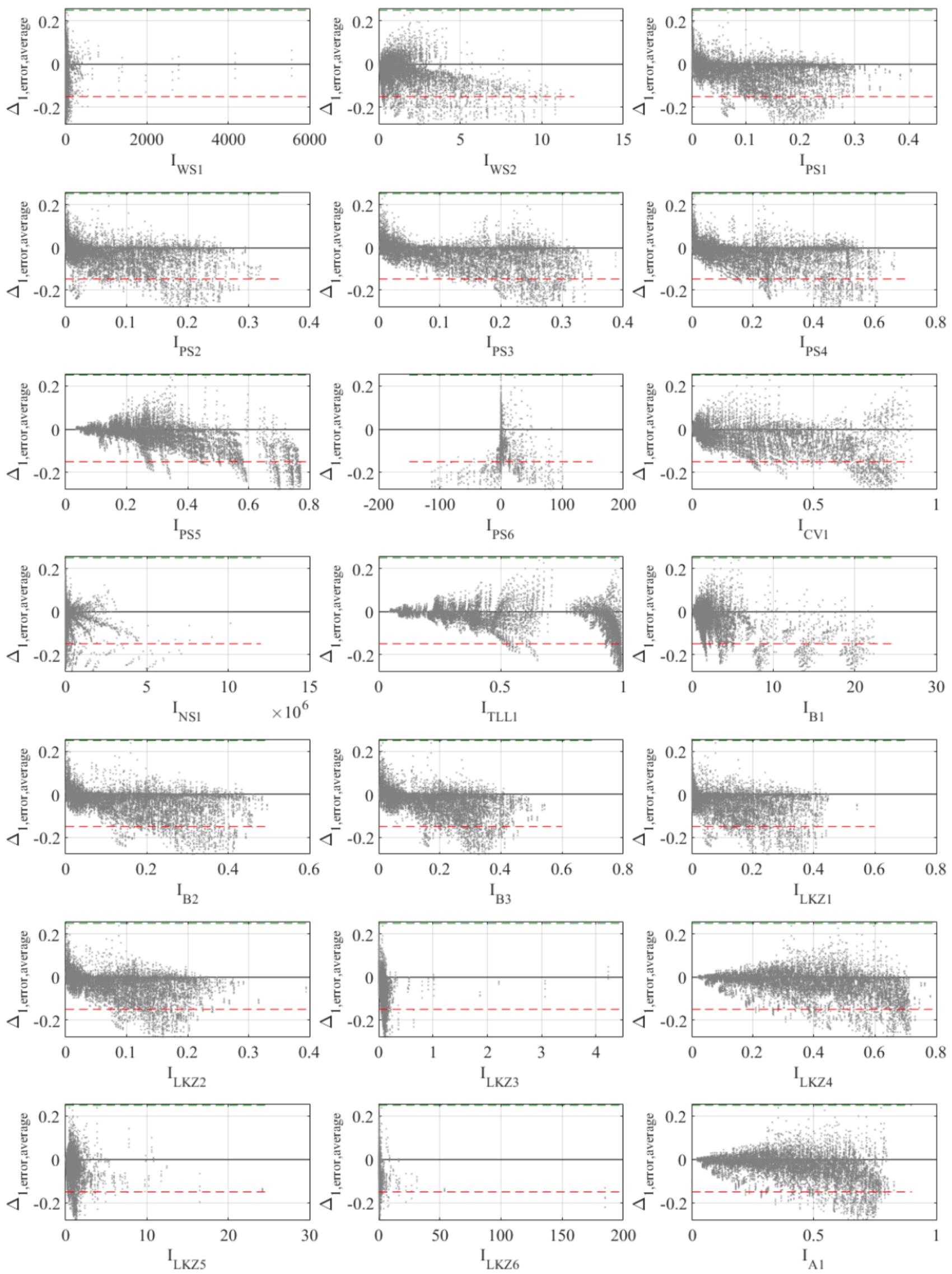

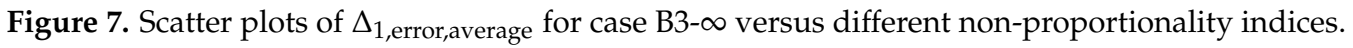



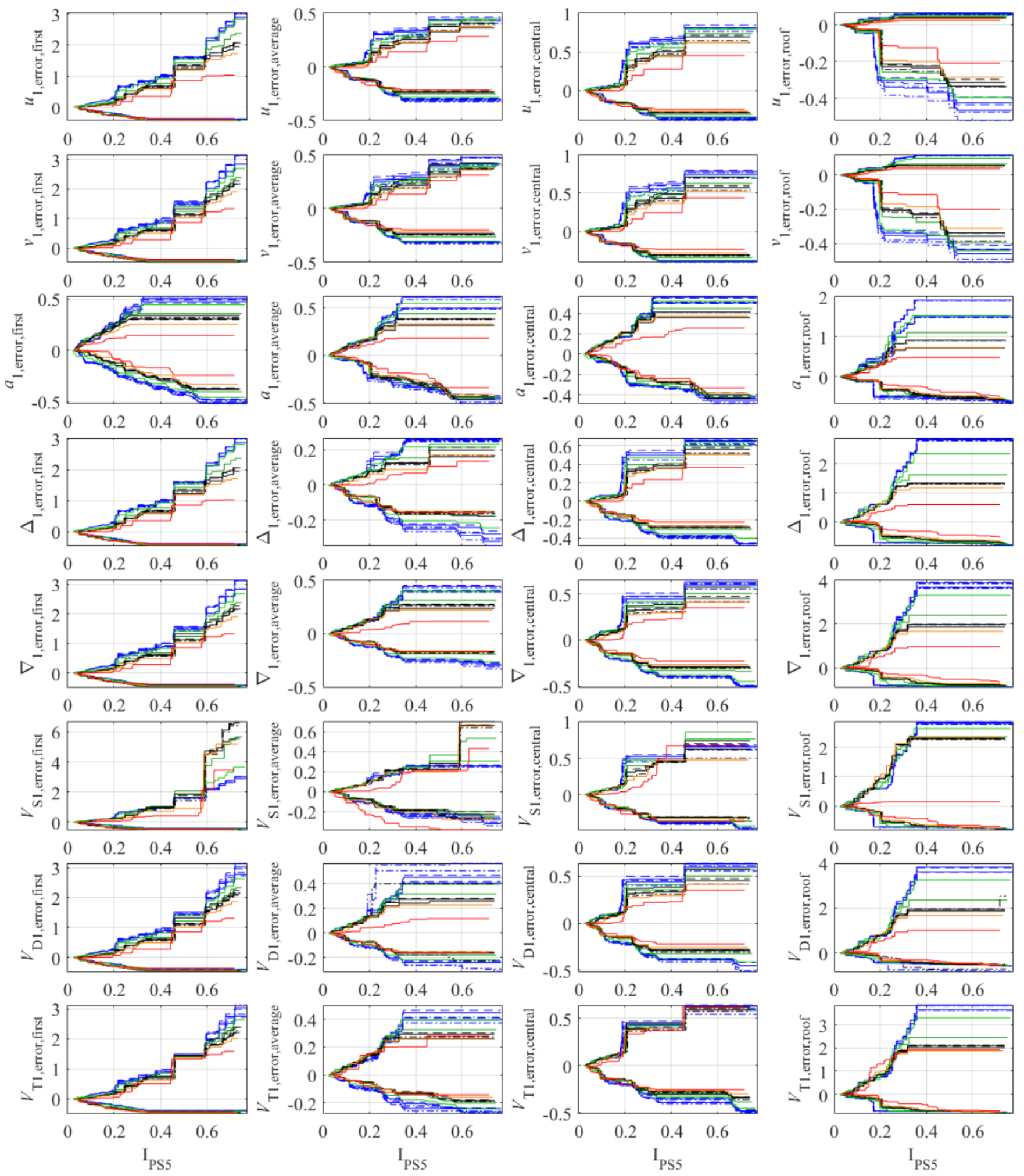
$-\mathrm{B} 0-\infty-\mathrm{C} 0-\infty$
B3 $-\infty$
C $3-\infty$
B5- $\infty$
C5- $\infty$
B3-10
$-\mathrm{B} 3-2-\mathrm{B} 0-1-\mathrm{B} 3-1$
C3-1
B5-1
B3-0.50 -
B3-0.10

Figure 8. THMA error (type 1) max and min envelope for all cases versus index $I_{P S 5}$. 

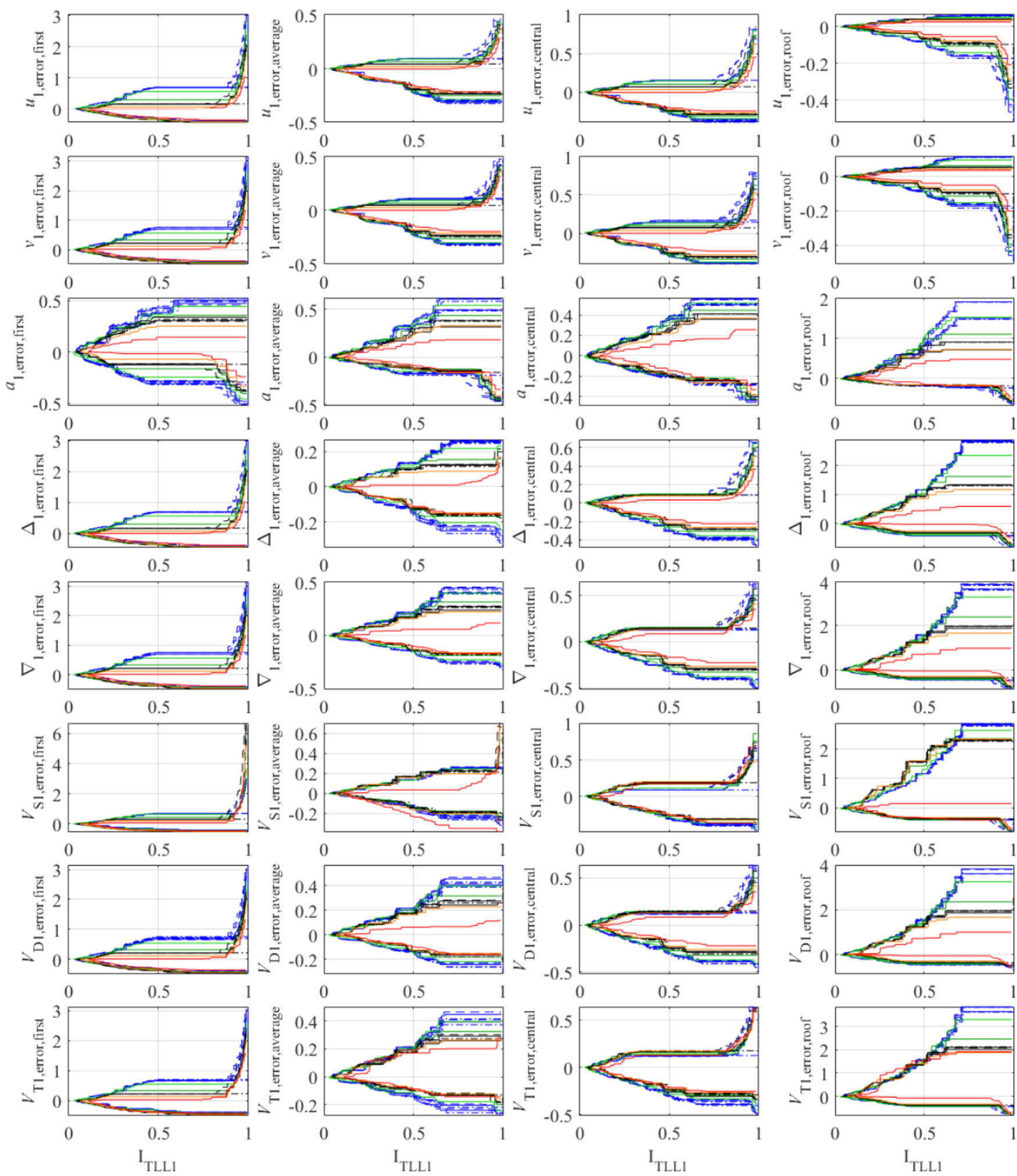

0.5
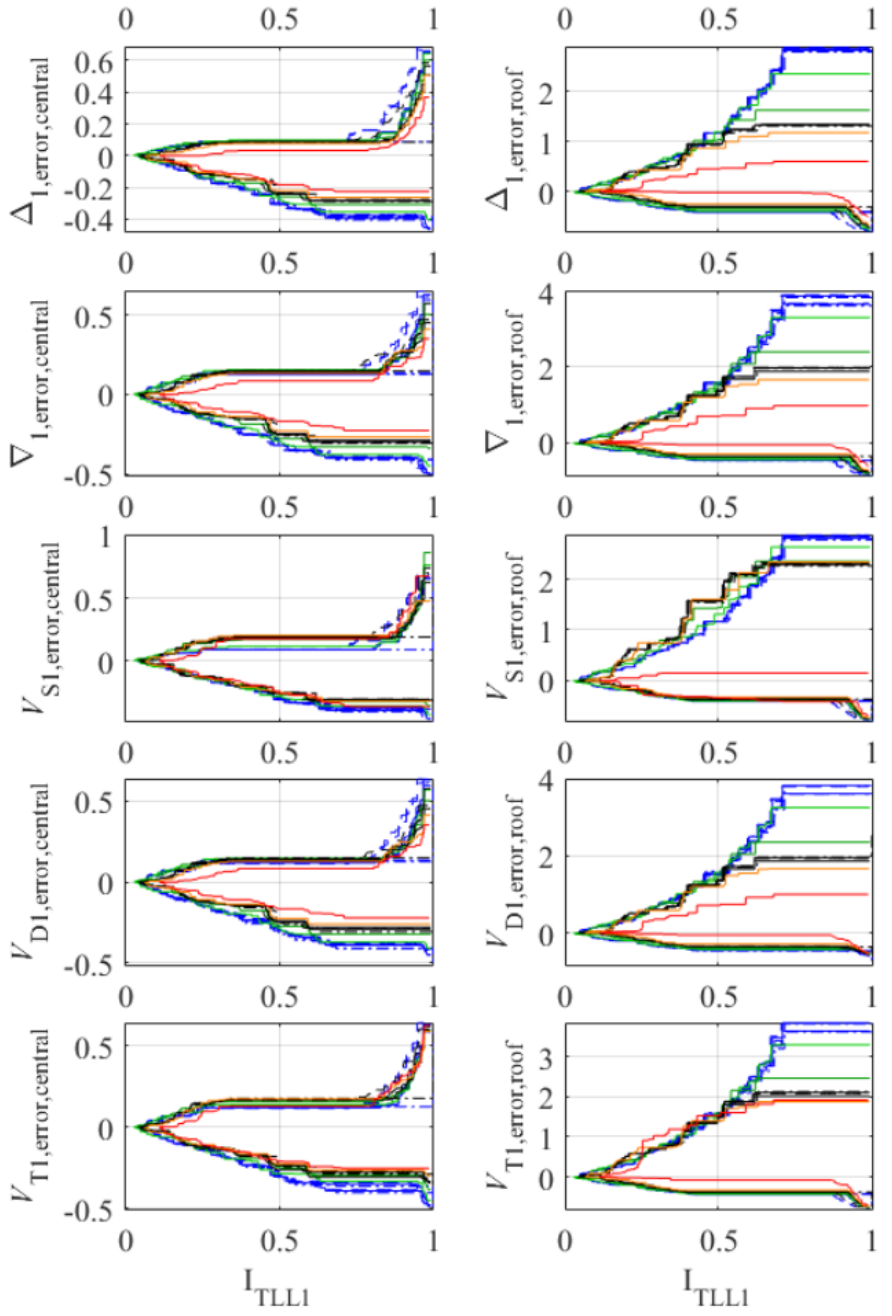

$-\mathrm{B} 0-$

\begin{abstract}
$\mathrm{C} 0-\infty$
B $3-\infty$

$\mathrm{C} 3-\infty$
\end{abstract}

B5- $\infty$

C5- $\infty$

B3-10

B3-0.10

Figure 9. THMA error (type 1) max and min envelope for all cases versus index $I_{T L L 1}$. 

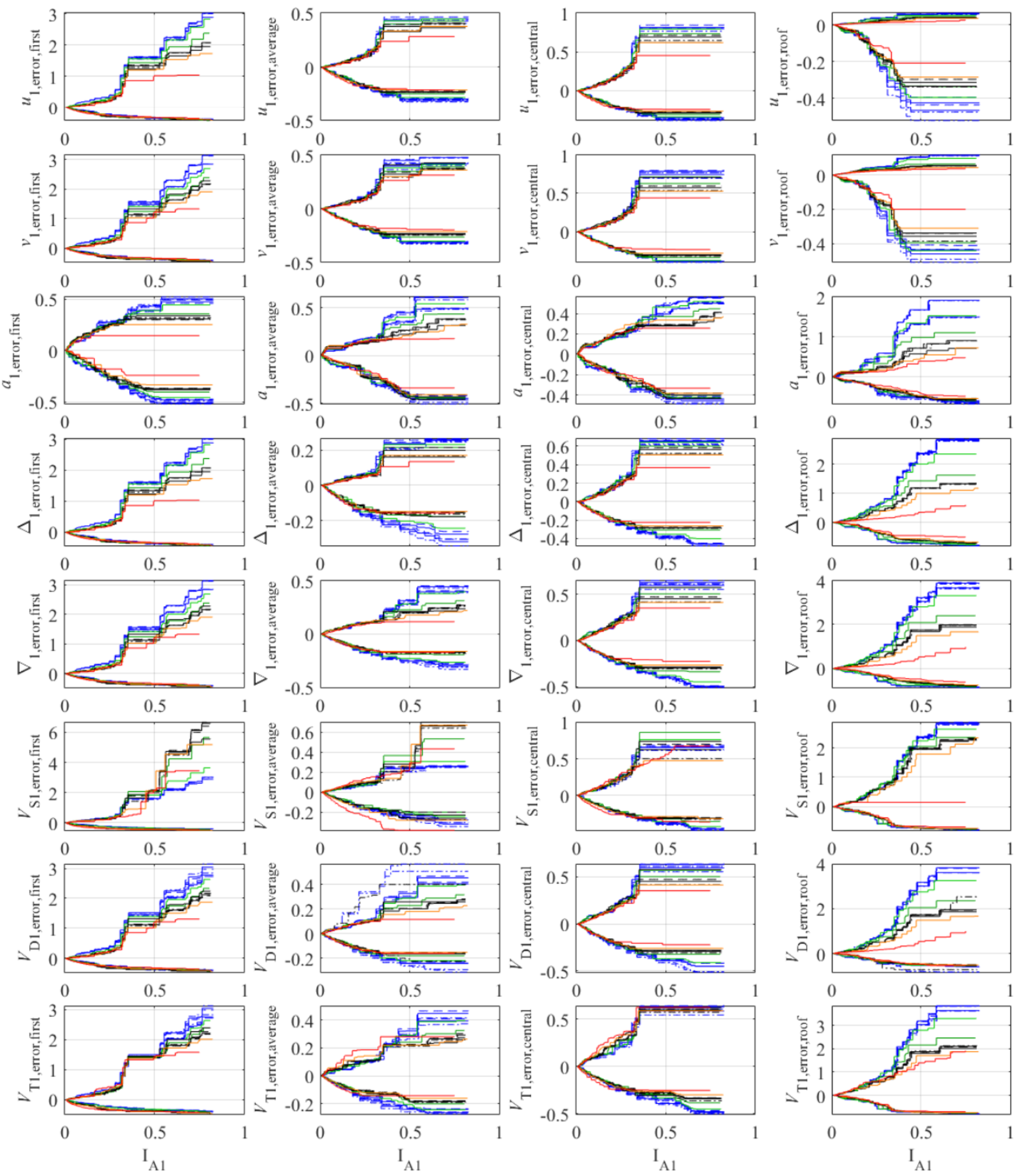

$$
\begin{array}{llll}
-\mathrm{B} 0-\infty-\mathrm{C} 0-\infty-\mathrm{B} 3-\infty-\mathrm{C} 3-\infty & \mathrm{B} 5-\infty-\mathrm{C} 5-\infty-\mathrm{B} 3-10 \\
-\mathrm{B} 3-2-\mathrm{B} 0-1-\mathrm{B} 3-1-\mathrm{C} 3-1-\mathrm{B} 5-1-\mathrm{B} 3-0.50-\mathrm{B} 3-0.10
\end{array}
$$

Figure 10. THMA error (type 1) max and min envelope for all cases versus index $I_{A 1}$.

For unconservative errors, the curves are, in general, more closely squeezed than for conservative errors. Thus, the distinction between frame-type and shear-type system is not 
relevant for un-conservative errors but is relevant for conservative errors, particularly at the roof. These observations are generally consistent with those of Section 3.

The max and min envelopes of all cases (outermost envelope curves) are used to obtain the maximum index value acceptable to limit the un-conservative and/or conservative THMA error; this information is listed in Table 7 (index $\left.I_{P S 5}\right)$, Table 8 (ITLL1), and Table $9\left(I_{A 1}\right)$, where no distinction has been made between frame-type and shear-type (and consequently the table is conservative for frame-type systems). For instance, to keep the un-conservative error $\Delta_{1 \text {,error }}$ below or equal -0.15 , the corresponding error row is located in Table 7 under header 'Error'; the value in this row on column under header 'first', sub-header ' $\Delta$ ' is 0.10 , indicating that $I_{P S 5}$ should be below or equal 0.10 to keep $\Delta_{1, \text { error,first }}$ below or equal to -0.15 ; other values are found in a similar way: 0.24 (average across stories), 0.12 (central story) or 0.20 (roof). The most limitative story is the first, and the corresponding value $(0.10)$ is found under the header 'ALL', sub-header ' $\triangle$ ', at the right side of the table. To keep the conservative error $\Delta_{1 \text {,error }}$ below +0.20 , the corresponding error row indicates that the index value should be limited to 0.13 (first story), 0.34 (average), 0.18 (central) or 0.10 (roof). The most limitative story is the roof and again the maximum index value (0.10) is listed under header 'ALL', sub-header ' $\Delta$ '. Thus, limiting the value of $I_{P S 5}$ to about 0.10 ensures that the max and min error fall within the $[-0.15 ;+0.20]$ range at all stories. A similar process with other variables renders corresponding limitative values of $I_{P S 5}: 0.10$ for $u_{1 \text {,error }}$ and $a_{1 \text {,error }} ; 0.08$ for $V_{\mathrm{S} 1 \text {,error }}$ and $V_{\mathrm{D} 1 \text {, error }}$. For $I_{T L L 1}$ and $I_{A 1}$ the rationale is the same, except that Table $8\left(I_{T L L 1}\right)$, or Table $9\left(I_{A 1}\right)$ should be used; the limiting value to keep THMA error within a $[-0.15 ;+0.20]$ range is $0.10\left(I_{T L L 1}\right)$ or $0.08\left(I_{A 1}\right)$. A system fulfilling any one of these three conditions $\left(I_{P S 5} \leq 0.10\right.$ or $I_{T L L 1} \leq 0.10$ or $\left.I_{A 1} \leq 0.08\right)$ will produce THMA errors within the $[-0.15 ;+0.20]$ range for the five relevant variables considered. It must be kept in mind, however, that to keep the THMA error under control the maximum index value is sufficient but not necessary, as shown by all points within boundary limits at scatter plots in Figure 7 . Information on the tables is limited to the maximum index value of the systems considered in the study.

Table 7. Maximum value of $I_{P S 5}$ to limit the max and min THMA error (error 1).

\begin{tabular}{|c|c|c|c|c|c|c|c|c|c|c|c|c|c|c|c|c|c|c|c|c|c|c|c|c|c|}
\hline \multirow{2}{*}{ Error } & \multicolumn{5}{|c|}{ First Story } & \multicolumn{5}{|c|}{ Average Across Stories } & \multicolumn{5}{|c|}{ Central Story } & \multicolumn{5}{|c|}{ Roof } & \multicolumn{5}{|c|}{ ALL } \\
\hline & $u$ & $a$ & $\Delta$ & $V_{\mathrm{S}}$ & $V_{\mathrm{D}}$ & $u$ & $a$ & $\Delta$ & $V_{S}$ & $V_{\mathrm{D}}$ & $u$ & $a$ & $\Delta$ & $V_{\mathrm{S}}$ & $V_{D}$ & $u$ & $a$ & $\Delta$ & $V_{\mathrm{S}}$ & $V_{\mathrm{D}}$ & $u$ & $a$ & $\Delta$ & $V_{\mathrm{S}}$ & $V_{\mathrm{D}}$ \\
\hline-0.90 & & & & & & & & & & & & & & & & & & & & 0.77 & & & & & 0.77 \\
\hline-0.85 & & & & & & & & & & & & & & & & & & 0.77 & 0.77 & 0.77 & & & 0.77 & 0.77 & 0.77 \\
\hline-0.80 & & & & & & & & & & & & & & & & & & 0.75 & 0.74 & 0.77 & & & 0.75 & 0.74 & 0.77 \\
\hline-0.75 & & & & & & & & & & & & & & & & & 0.77 & 0.73 & 0.72 & 0.77 & & 0.77 & 0.73 & 0.72 & 0.77 \\
\hline-0.70 & & & & & & & & & & & & & & & & & 0.77 & 0.72 & 0.49 & 0.77 & & 0.77 & 0.72 & 0.49 & 0.77 \\
\hline-0.65 & & & & & & & & & & & & & & & & & 0.77 & 0.72 & 0.45 & 0.77 & & 0.77 & 0.72 & 0.45 & 0.77 \\
\hline-0.60 & & & & & & & & & & & & & & & 0.77 & & 0.74 & 0.72 & 0.45 & 0.75 & & 0.74 & 0.72 & 0.45 & 0.75 \\
\hline-0.55 & & 0.77 & & & & & 0.77 & & & & & 0.77 & 0.77 & 0.77 & 0.77 & 0.77 & 0.72 & 0.71 & 0.41 & 0.74 & 0.77 & 0.72 & 0.71 & 0.41 & 0.74 \\
\hline-0.50 & & 0.75 & & & 0.77 & & 0.77 & & & & & 0.77 & 0.76 & 0.76 & 0.77 & 0.77 & 0.64 & 0.62 & 0.41 & 0.55 & 0.77 & 0.64 & 0.62 & 0.41 & 0.55 \\
\hline-0.45 & 0.77 & 0.74 & 0.77 & 0.77 & 0.77 & & 0.72 & & & & 0.77 & 0.74 & 0.75 & 0.75 & 0.75 & 0.77 & 0.62 & 0.45 & 0.33 & 0.49 & 0.77 & 0.62 & 0.45 & 0.33 & 0.49 \\
\hline-0.40 & 0.32 & 0.73 & 0.32 & 0.32 & 0.35 & 0.77 & $0.7 \overline{2}$ & 0.77 & 0.77 & & 0.75 & 0.72 & 0.75 & 0.74 & 0.75 & 0.75 & 0.46 & 0.45 & 0.22 & 0.42 & 0.32 & 0.46 & 0.32 & 0.22 & 0.35 \\
\hline-0.35 & 0.27 & 0.72 & 0.27 & 0.27 & 0.27 & 0.77 & 0.51 & 0.77 & 0.77 & 0.77 & 0.74 & 0.51 & 0.74 & 0.32 & 0.74 & 0.74 & 0.41 & 0.28 & 0.21 & 0.30 & 0.27 & 0.41 & 0.27 & 0.21 & 0.27 \\
\hline-0.30 & 0.24 & 0.72 & 0.24 & 0.24 & 0.25 & 0.75 & 0.48 & 0.76 & 0.76 & 0.77 & 0.34 & 0.48 & 0.72 & 0.26 & 0.72 & 0.72 & 0.40 & 0.23 & 0.21 & 0.28 & 0.24 & 0.40 & 0.23 & 0.21 & 0.25 \\
\hline-0.25 & 0.19 & 0.44 & 0.19 & 0.19 & 0.20 & 0.34 & 0.45 & 0.75 & 0.57 & 0.75 & 0.25 & 0.26 & 0.26 & 0.23 & 0.32 & 0.46 & 0.23 & 0.21 & 0.21 & 0.24 & 0.19 & 0.23 & 0.19 & 0.19 & 0.20 \\
\hline-0.20 & 0.15 & 0.28 & 0.15 & 0.15 & 0.15 & 0.25 & 0.28 & 0.73 & 0.30 & 0.32 & 0.21 & 0.22 & 0.24 & 0.16 & 0.24 & 0.45 & 0.21 & 0.21 & 0.18 & 0.20 & 0.15 & 0.21 & 0.15 & 0.15 & 0.15 \\
\hline-0.15 & 0.10 & 0.24 & 0.10 & 0.10 & 0.11 & 0.18 & 0.21 & 0.24 & 0.23 & 0.24 & 0.12 & 0.18 & 0.12 & 0.12 & 0.11 & 0.21 & 0.19 & 0.20 & 0.14 & 0.20 & 0.10 & 0.18 & 0.10 & 0.10 & 0.11 \\
\hline-0.10 & 0.06 & 0.19 & 0.06 & 0.06 & 0.06 & 0.11 & 0.16 & 0.12 & 0.12 & 0.11 & 0.11 & 0.14 & 0.10 & 0.10 & 0.09 & 0.21 & 0.10 & 0.19 & 0.11 & 0.19 & 0.06 & 0.10 & 0.06 & 0.06 & 0.06 \\
\hline-0.05 & 0.04 & 0.07 & 0.04 & 0.04 & 0.04 & 0.04 & 0.04 & 0.04 & 0.04 & 0.04 & 0.04 & 0.04 & 0.04 & 0.04 & 0.04 & 0.05 & 0.05 & 0.17 & 0.05 & 0.09 & 0.04 & 0.04 & 0.04 & 0.04 & 0.04 \\
\hline 0 & 0 & 0 & 0 & 0 & 0 & 0 & 0 & 0 & 0 & 0 & 0 & 0 & 0 & 0 & 0 & 0 & 0 & 0 & 0 & 0 & 0 & 0 & 0 & 0 & 0 \\
\hline 0.05 & 0.04 & 0.04 & 0.04 & 0.04 & 0.04 & 0.16 & 0.06 & 0.13 & 0.11 & 0.08 & 0.10 & 0.05 & 0.07 & 0.07 & 0.07 & 0.26 & 0.05 & 0.04 & 0.04 & 0.04 & 0.04 & 0.04 & 0.04 & 0.04 & 0.04 \\
\hline 0.10 & 0.08 & 0.07 & 0.08 & 0.08 & 0.08 & 0.18 & 0.10 & 0.19 & 0.19 & 0.15 & 0.17 & 0.10 & 0.17 & 0.15 & 0.13 & 0.72 & 0.07 & 0.05 & 0.05 & 0.05 & 0.08 & 0.07 & 0.05 & 0.05 & 0.05 \\
\hline 0.15 & 0.10 & 0.11 & 0.10 & 0.10 & 0.10 & 0.19 & 0.15 & 0.20 & 0.20 & 0.18 & 0.18 & 0.15 & 0.18 & 0.18 & 0.17 & & 0.10 & 0.08 & 0.05 & 0.06 & 0.10 & 0.10 & 0.08 & 0.05 & 0.06 \\
\hline 0.20 & 0.13 & 0.15 & 0.13 & 0.13 & 0.13 & 0.20 & 0.22 & 0.34 & 0.27 & 0.19 & 0.18 & 0.17 & 0.18 & 0.18 & 0.18 & & 0.10 & 0.10 & 0.08 & 0.08 & 0.13 & 0.10 & 0.10 & 0.08 & 0.08 \\
\hline 0.25 & 0.18 & 0.17 & 0.18 & 0.18 & 0.18 & 0.20 & 0.22 & 0.36 & 0.36 & 0.19 & 0.18 & 0.23 & 0.18 & 0.18 & 0.18 & & 0.10 & 0.10 & 0.08 & 0.10 & 0.18 & 0.10 & 0.10 & 0.08 & 0.10 \\
\hline 0.30 & 0.18 & 0.17 & 0.18 & 0.18 & 0.18 & 0.20 & 0.22 & 0.36 & 0.36 & 0.19 & 0.18 & 0.23 & 0.18 & 0.18 & 0.18 & & 0.10 & 0.10 & 0.08 & 0.10 & 0.18 & 0.10 & 0.10 & 0.08 & 0.10 \\
\hline 0.35 & 0.19 & 0.22 & 0.19 & 0.19 & 0.20 & 0.22 & 0.24 & 0.72 & 0.46 & 0.19 & 0.19 & 0.24 & 0.19 & 0.19 & 0.19 & & 0.11 & 0.10 & 0.10 & 0.10 & 0.19 & 0.11 & 0.10 & 0.10 & 0.10 \\
\hline 0.40 & 0.20 & 0.23 & 0.20 & 0.20 & 0.20 & 0.30 & 0.25 & & 0.46 & 0.21 & 0.19 & 0.25 & 0.19 & 0.19 & 0.19 & & 0.14 & 0.10 & 0.10 & 0.10 & 0.19 & 0.14 & 0.10 & 0.10 & 0.10 \\
\hline 0.45 & 0.20 & 0.24 & 0.20 & 0.20 & 0.20 & 0.46 & 0.25 & & 0.59 & 0.23 & 0.20 & 0.27 & 0.19 & 0.19 & 0.19 & & 0.14 & 0.10 & 0.10 & 0.10 & 0.20 & 0.14 & 0.10 & 0.10 & 0.10 \\
\hline 0.50 & 0.20 & 0.31 & 0.20 & 0.20 & 0.20 & 0.46 & 0.30 & & 0.59 & 0.23 & 0.20 & 0.31 & 0.19 & 0.19 & 0.19 & & 0.14 & 0.13 & 0.11 & 0.10 & 0.20 & 0.14 & 0.13 & 0.11 & 0.10 \\
\hline 0.55 & 0.20 & 0.32 & 0.20 & 0.20 & 0.20 & 0.72 & 0.32 & & 0.59 & 0.23 & 0.20 & 0.32 & 0.19 & 0.19 & 0.22 & & 0.18 & 0.13 & 0.13 & 0.10 & 0.20 & 0.18 & 0.13 & 0.13 & 0.10 \\
\hline 0.60 & 0.22 & 0.72 & 0.22 & 0.22 & 0.22 & & 0.34 & & 0.59 & 0.72 & 0.22 & 0.72 & 0.46 & 0.38 & 0.46 & & 0.22 & 0.16 & 0.16 & 0.13 & 0.22 & 0.22 & 0.16 & 0.16 & 0.13 \\
\hline 0.65 & 0.22 & & 0.22 & 0.22 & 0.27 & & 0.72 & & 0.59 & & 0.30 & & 0.46 & 0.38 & 0.72 & & 0.22 & 0.21 & 0.17 & 0.15 & 0.22 & 0.22 & 0.21 & 0.17 & 0.15 \\
\hline 0.70 & 0.27 & & 0.27 & 0.27 & 0.27 & & & & 0.59 & & 0.40 & & 0.72 & 0.46 & & & 0.22 & 0.21 & 0.17 & 0.16 & 0.27 & 0.22 & 0.21 & 0.17 & 0.16 \\
\hline 0.75 & 0.27 & & 0.27 & 0.27 & 0.30 & & & & 0.72 & & 0.46 & & & 0.46 & & & 0.22 & 0.23 & 0.17 & 0.16 & 0.27 & 0.22 & 0.23 & 0.17 & 0.16 \\
\hline 0.80 & 0.30 & & 0.30 & 0.30 & 0.30 & & & & & & 0.46 & & & 0.46 & & & 0.24 & 0.24 & 0.18 & 0.18 & 0.30 & 0.24 & 0.24 & 0.18 & 0.18 \\
\hline 0.85 & 0.30 & & 0.30 & 0.30 & 0.38 & & & & & & 0.72 & & & 0.46 & & & 0.24 & 0.24 & & 0.21 & 0.30 & 0.24 & 0.24 & 0.30 & 0.21 \\
\hline 0.90 & 0.38 & & 0.38 & 0.33 & 0.38 & & & & & & & & & 0.72 & & & 0.24 & 0.24 & & 0.21 & 0.38 & 0.24 & 0.24 & 0.33 & 0.21 \\
\hline 0.95 & & & & 0.34 & 0.40 & & & & & & & & & & & & 0.24 & 0.25 & & 0.22 & & 0.24 & 0.25 & 0.34 & 0.22 \\
\hline 1.00 & & & & 0.36 & 0.45 & & & & & & & & & & & & & & & 0.23 & & & & 0.36 & 0.23 \\
\hline
\end{tabular}


Table 8. Maximum value of $I_{T L L 1}$ to limit the max and min THMA error (error 1).

\begin{tabular}{|c|c|c|c|c|c|c|c|c|c|c|c|c|c|c|c|c|c|c|c|c|c|c|c|c|c|}
\hline \multirow{2}{*}{ Error } & \multicolumn{5}{|c|}{ First Story } & \multicolumn{5}{|c|}{ Average Across Stories } & \multicolumn{5}{|c|}{ Central Story } & \multicolumn{5}{|c|}{ Roof } & \multicolumn{5}{|c|}{ ALL } \\
\hline & $u$ & $a$ & $\Delta$ & $V_{\mathrm{S}}$ & $V_{\mathrm{D}}$ & $u$ & $a$ & $\Delta$ & $V_{\mathrm{S}}$ & $V_{\mathrm{D}}$ & $u$ & $a$ & $\Delta$ & $V_{\mathrm{S}}$ & $V_{\mathrm{D}}$ & $u$ & $a$ & $\Delta$ & $V_{\mathrm{S}}$ & $V_{\mathrm{D}}$ & $u$ & $a$ & $\Delta$ & $V_{\mathrm{S}}$ & $V_{\mathrm{D}}$ \\
\hline-0.75 & & & 1.00 & 1.00 & & & & 1.00 & 1.00 & & & & 1.00 & 1.00 & & & & 1.00 & 1.00 & & & & 1.00 & 1.00 & \\
\hline-0.70 & & & 1.00 & 1.00 & & & & 1.00 & 1.00 & & & & 1.00 & 1.00 & & & & 1.00 & 1.00 & & & & 1.00 & 1.00 & \\
\hline-0.65 & & & 1.00 & 1.00 & & & & 1.00 & 1.00 & & & & 1.00 & 1.00 & & & & 1.00 & 1.00 & & & & 1.00 & 1.00 & \\
\hline-0.60 & & 1.00 & 1.00 & 1.00 & & & 1.00 & 1.00 & 1.00 & & & 1.00 & 1.00 & 1.00 & & & 1.00 & 1.00 & 1.00 & & & 1.00 & 1.00 & 1.00 & \\
\hline-0.55 & & 1.00 & 1.00 & 1.00 & 1.00 & & 1.00 & 1.00 & 1.00 & 1.00 & & 1.00 & 1.00 & 1.00 & 1.00 & & 1.00 & 1.00 & 1.00 & 1.00 & & 1.00 & 1.00 & 1.00 & 1.00 \\
\hline-0.50 & & 1.00 & 1.00 & 1.00 & 1.00 & & 1.00 & 1.00 & 1.00 & 1.00 & & 1.00 & 1.00 & 1.00 & 1.00 & & 1.00 & 1.00 & 1.00 & 1.00 & & 1.00 & 1.00 & 1.00 & 1.00 \\
\hline-0.45 & 1.00 & 1.00 & 1.00 & 1.00 & 0.99 & 1.00 & 1.00 & 1.00 & 1.00 & 0.99 & 1.00 & 1.00 & 1.00 & 1.00 & 0.99 & 1.00 & 1.00 & 1.00 & 1.00 & 0.99 & 1.00 & 1.00 & 1.00 & 1.00 & 0.99 \\
\hline-0.40 & 0.66 & 1.00 & 0.66 & 0.59 & 0.73 & 0.66 & 1.00 & 0.66 & 0.59 & 0.73 & 0.66 & 1.00 & 0.66 & 0.59 & 0.73 & 0.66 & 1.00 & 0.66 & 0.59 & 0.73 & 0.66 & 1.00 & 0.66 & 0.59 & 0.73 \\
\hline-0.35 & 0.49 & 1.00 & 0.49 & 0.35 & 0.55 & 0.49 & 1.00 & 0.49 & 0.35 & 0.55 & 0.49 & 1.00 & 0.49 & 0.35 & 0.55 & 0.49 & 1.00 & 0.49 & 0.35 & 0.55 & 0.49 & 1.00 & 0.49 & 0.35 & 0.55 \\
\hline-0.30 & 0.38 & 0.94 & 0.38 & 0.30 & 0.37 & 0.38 & 0.94 & 0.38 & 0.30 & 0.37 & 0.38 & 0.94 & 0.38 & 0.30 & 0.37 & 0.38 & 0.94 & 0.38 & 0.30 & 0.37 & 0.38 & 0.94 & 0.38 & 0.30 & 0.37 \\
\hline-0.25 & 0.29 & 0.62 & 0.29 & 0.23 & 0.29 & 0.29 & 0.62 & 0.29 & 0.23 & 0.29 & 0.29 & 0.62 & 0.29 & 0.23 & 0.29 & 0.29 & 0.62 & 0.29 & 0.23 & 0.29 & 0.29 & 0.62 & 0.29 & 0.23 & 0.29 \\
\hline-0.20 & 0.25 & 0.51 & 0.25 & 0.23 & 0.25 & 0.25 & 0.51 & 0.25 & 0.23 & 0.25 & 0.25 & 0.51 & 0.25 & 0.23 & 0.25 & 0.25 & 0.51 & 0.25 & 0.23 & 0.25 & 0.25 & 0.51 & 0.25 & 0.23 & 0.25 \\
\hline-0.15 & 0.20 & 0.39 & 0.20 & 0.15 & 0.20 & 0.20 & 0.39 & 0.20 & 0.15 & 0.20 & 0.20 & 0.39 & 0.20 & 0.15 & 0.20 & 0.20 & 0.39 & 0.20 & 0.15 & 0.20 & 0.20 & 0.39 & 0.20 & 0.15 & 0.20 \\
\hline-0.10 & 0.13 & 0.26 & 0.13 & 0.13 & 0.14 & 0.13 & 0.26 & 0.13 & 0.13 & 0.14 & 0.13 & 0.26 & 0.13 & 0.13 & 0.14 & 0.13 & 0.26 & 0.13 & 0.13 & 0.14 & 0.13 & 0.26 & 0.13 & 0.13 & 0.14 \\
\hline-0.05 & 0.04 & 0.06 & 0.04 & 0.04 & 0.05 & 0.04 & 0.06 & 0.04 & 0.04 & 0.05 & 0.04 & 0.06 & 0.04 & 0.04 & 0.05 & 0.04 & 0.06 & 0.04 & 0.04 & 0.05 & 0.04 & 0.06 & 0.04 & 0.04 & 0.05 \\
\hline 0 & 0 & 0 & 0 & 0 & 0 & 0 & 0 & 0 & 0 & 0 & 0 & 0 & 0 & 0 & 0 & 0 & 0 & 0 & 0 & 0 & 0 & 0 & 0 & 0 & 0 \\
\hline 0.05 & 0.07 & 0.05 & 0.05 & 0.05 & 0.05 & 0.07 & 0.05 & 0.05 & 0.05 & 0.05 & 0.07 & 0.05 & 0.05 & 0.05 & 0.05 & 0.07 & 0.05 & 0.05 & 0.05 & 0.05 & 0.07 & 0.05 & 0.05 & 0.05 & 0.05 \\
\hline 0.10 & 0.11 & 0.10 & 0.07 & 0.07 & 0.07 & 0.11 & 0.10 & 0.07 & 0.07 & 0.07 & 0.11 & 0.10 & 0.07 & 0.07 & 0.07 & 0.11 & 0.10 & 0.07 & 0.07 & 0.07 & 0.11 & 0.10 & 0.07 & 0.07 & 0.07 \\
\hline 0.15 & 0.14 & 0.15 & 0.10 & 0.09 & 0.10 & 0.14 & 0.15 & 0.10 & 0.09 & 0.10 & 0.14 & 0.15 & 0.10 & 0.09 & 0.10 & 0.14 & 0.15 & 0.10 & 0.09 & 0.10 & 0.14 & 0.15 & 0.10 & 0.09 & 0.10 \\
\hline 0.20 & 0.16 & 0.20 & 0.14 & 0.12 & 0.10 & 0.16 & 0.20 & 0.14 & 0.12 & 0.10 & 0.16 & 0.20 & 0.14 & 0.12 & 0.10 & 0.16 & 0.20 & 0.14 & 0.12 & 0.10 & 0.16 & 0.20 & 0.14 & 0.12 & 0.10 \\
\hline 0.25 & 0.23 & 0.23 & 0.16 & 0.16 & 0.14 & 0.23 & 0.23 & 0.16 & 0.16 & 0.14 & 0.23 & 0.23 & 0.16 & 0.16 & 0.14 & 0.23 & 0.23 & 0.16 & 0.16 & 0.14 & 0.23 & 0.23 & 0.16 & 0.16 & 0.14 \\
\hline 0.30 & 0.23 & 0.23 & 0.16 & 0.16 & 0.14 & 0.23 & 0.23 & 0.16 & 0.16 & 0.14 & 0.23 & 0.23 & 0.16 & 0.16 & 0.14 & 0.23 & 0.23 & 0.16 & 0.16 & 0.14 & 0.23 & 0.23 & 0.16 & 0.16 & 0.14 \\
\hline 0.35 & 0.25 & 0.24 & 0.19 & 0.16 & 0.16 & 0.25 & 0.24 & 0.19 & 0.16 & 0.16 & 0.25 & 0.24 & 0.19 & 0.16 & 0.16 & 0.25 & 0.24 & 0.19 & 0.16 & 0.16 & 0.25 & 0.24 & 0.19 & 0.16 & 0.16 \\
\hline 0.40 & 0.26 & 0.27 & 0.19 & 0.17 & 0.19 & 0.26 & 0.27 & 0.19 & 0.17 & 0.19 & 0.26 & 0.27 & 0.19 & 0.17 & 0.19 & 0.26 & 0.27 & 0.19 & 0.17 & 0.19 & 0.26 & 0.27 & 0.19 & 0.17 & 0.19 \\
\hline 0.45 & 0.30 & 0.27 & 0.21 & 0.19 & 0.19 & 0.30 & 0.27 & 0.21 & 0.19 & 0.19 & 0.30 & 0.27 & 0.21 & 0.19 & 0.19 & 0.30 & 0.27 & 0.21 & 0.19 & 0.19 & 0.30 & 0.27 & 0.21 & 0.19 & 0.19 \\
\hline 0.50 & 0.31 & 0.28 & 0.25 & 0.19 & 0.19 & 0.31 & 0.28 & 0.25 & 0.19 & 0.19 & 0.31 & 0.28 & 0.25 & 0.19 & 0.19 & 0.31 & 0.28 & 0.25 & 0.19 & 0.19 & 0.31 & 0.28 & 0.25 & 0.19 & 0.19 \\
\hline 0.55 & 0.32 & 0.30 & 0.27 & 0.19 & 0.21 & 0.32 & 0.30 & 0.27 & 0.19 & 0.21 & 0.32 & 0.30 & 0.27 & 0.19 & 0.21 & 0.32 & 0.30 & 0.27 & 0.19 & 0.21 & 0.32 & 0.30 & 0.27 & 0.19 & 0.21 \\
\hline 0.60 & 0.40 & 0.36 & 0.31 & 0.21 & 0.26 & 0.40 & 0.36 & 0.31 & 0.21 & 0.26 & 0.40 & 0.36 & 0.31 & 0.21 & 0.26 & 0.40 & 0.36 & 0.31 & 0.21 & 0.26 & 0.40 & 0.36 & 0.31 & 0.21 & 0.26 \\
\hline 0.65 & 0.42 & 0.40 & 0.32 & 0.24 & 0.28 & 0.42 & 0.40 & 0.32 & 0.24 & 0.28 & 0.42 & 0.40 & 0.32 & 0.24 & 0.28 & 0.42 & 0.40 & 0.32 & 0.24 & 0.28 & 0.42 & 0.40 & 0.32 & 0.24 & 0.28 \\
\hline 0.70 & 0.47 & 0.40 & 0.35 & 0.24 & 0.30 & 0.47 & 0.40 & 0.35 & 0.24 & 0.30 & 0.47 & 0.40 & 0.35 & 0.24 & 0.30 & 0.47 & 0.40 & 0.35 & 0.24 & 0.30 & 0.47 & 0.40 & 0.35 & 0.24 & 0.30 \\
\hline 0.75 & 0.89 & 0.47 & 0.36 & 0.31 & 0.31 & 0.89 & 0.47 & 0.36 & 0.31 & 0.31 & 0.89 & 0.47 & 0.36 & 0.31 & 0.31 & 0.89 & 0.47 & 0.36 & 0.31 & 0.31 & 0.89 & 0.47 & 0.36 & 0.31 & 0.31 \\
\hline 0.80 & 0.89 & 0.47 & 0.39 & 0.33 & 0.31 & 0.89 & 0.47 & 0.39 & 0.33 & 0.31 & 0.89 & 0.47 & 0.39 & 0.33 & 0.31 & 0.89 & 0.47 & 0.39 & 0.33 & 0.31 & 0.89 & 0.47 & 0.39 & 0.33 & 0.31 \\
\hline 0.8 & 0.89 & 0.48 & 0.40 & 0.36 & 0.34 & 0.89 & 0.48 & 0.40 & 0.36 & 0.34 & 0.89 & 0.48 & 0.40 & 0.36 & 0.34 & 0.89 & 0.48 & 0.40 & 0.36 & 0.34 & 0.89 & 0.48 & 0.40 & 0.36 & 0.34 \\
\hline 0.90 & 0.91 & 0.49 & 0.40 & 0.37 & 0.34 & 0.91 & 0.49 & 0.40 & 0.37 & 0.34 & 0.91 & 0.49 & 0.40 & 0.37 & 0.34 & 0.91 & 0.49 & 0.40 & 0.37 & 0.34 & 0.91 & 0.49 & 0.40 & 0.37 & 0.34 \\
\hline 0.95 & & 0.49 & 0.40 & 0.37 & 0.36 & & 0.49 & 0.40 & 0.37 & 0.36 & & 0.49 & 0.40 & 0.37 & 0.36 & & 0.49 & 0.40 & 0.37 & 0.36 & & 0.49 & 0.40 & 0.37 & 0.36 \\
\hline 1.00 & & & 0.42 & & 0.92 & & & 0.42 & & 0.92 & & & 0.42 & & 0.92 & & & 0.42 & & 0.92 & & & 0.42 & & 0.92 \\
\hline
\end{tabular}

Table 9. Maximum value of $I_{A 1}$ to limit the max and min THMA error (error 1).

\begin{tabular}{|c|c|c|c|c|c|c|c|c|c|c|c|c|c|c|c|c|c|c|c|c|c|c|c|c|c|}
\hline \multirow{2}{*}{ Error } & \multicolumn{5}{|c|}{ First story } & \multicolumn{5}{|c|}{ Average across stories } & \multicolumn{5}{|c|}{ Central story } & \multicolumn{5}{|c|}{ Roof } & \multicolumn{5}{|c|}{ ALL } \\
\hline & $u$ & $a$ & $\Delta$ & $V_{\mathrm{S}}$ & $V_{\mathrm{D}}$ & $u$ & $a$ & $\Delta$ & $V_{\mathrm{S}}$ & $V_{\mathrm{D}}$ & $u$ & $a$ & $\Delta$ & $V_{\mathrm{S}}$ & $V_{\mathrm{D}}$ & $u$ & $a$ & $\Delta$ & $V_{\mathrm{S}}$ & $V_{\mathrm{D}}$ & $u$ & $a$ & $\Delta$ & $V_{\mathrm{S}}$ & $V_{\mathrm{D}}$ \\
\hline-0.85 & & & & & & & & & & & & & & & & & & 0.83 & 0.83 & & & & 0.83 & 0.83 & \\
\hline-0.80 & & & & & & & & & & & & & & & & & & 0.82 & 0.82 & & & & 0.82 & 0.82 & \\
\hline-0.75 & & & & & & & & & & & & & & & & & & 0.82 & 0.75 & & & & 0.82 & 0.75 & \\
\hline-0.70 & & & & & & & & & & & & & & & & & & 0.75 & 0.39 & & & & 0.75 & 0.39 & \\
\hline-0.65 & & & & & & & & & & & & & & & & & 0.83 & 0.75 & 0.35 & 0.83 & & 0.83 & 0.75 & 0.35 & 0.83 \\
\hline-0.60 & & & & & & & & & & & & & & & & & 0.82 & 0.75 & 0.34 & 0.82 & & 0.82 & 0.75 & 0.34 & 0.82 \\
\hline-0.55 & & 0.83 & & & & & & & & & & & 0.83 & 0.83 & & & 0.75 & 0.71 & 0.33 & 0.81 & & 0.75 & 0.71 & 0.33 & 0.81 \\
\hline-0.50 & & 0.82 & & & & & 0.83 & & & & & 0.83 & 0.82 & 0.82 & 0.83 & & 0.53 & 0.52 & 0.32 & 0.50 & & 0.53 & 0.52 & 0.32 & 0.50 \\
\hline-0.45 & 0.83 & 0.82 & 0.83 & 0.83 & 0.83 & & 0.75 & & & & 0.83 & 0.82 & 0.82 & 0.82 & 0.82 & 0.83 & 0.49 & 0.34 & 0.32 & 0.38 & 0.83 & 0.49 & 0.34 & 0.32 & 0.38 \\
\hline-0.40 & 0.61 & 0.82 & 0.61 & 0.54 & 0.68 & & 0.75 & & & & 0.82 & 0.75 & 0.82 & 0.82 & 0.82 & 0.82 & 0.44 & 0.33 & 0.27 & 0.34 & 0.61 & 0.44 & 0.33 & 0.27 & 0.34 \\
\hline-0.35 & 0.40 & 0.75 & 0.40 & 0.40 & 0.48 & 0.83 & 0.41 & 0.83 & 0.83 & & 0.82 & 0.42 & 0.82 & 0.68 & 0.82 & 0.82 & 0.38 & 0.28 & 0.27 & 0.28 & 0.40 & 0.38 & 0.28 & 0.27 & 0.28 \\
\hline-0.30 & 0.26 & 0.75 & 0.26 & 0.26 & 0.29 & 0.82 & 0.37 & 0.82 & 0.81 & 0.83 & 0.48 & 0.37 & 0.75 & 0.29 & 0.75 & 0.75 & 0.34 & 0.23 & 0.22 & 0.24 & 0.26 & 0.34 & 0.23 & 0.22 & 0.24 \\
\hline-0.25 & 0.18 & 0.32 & 0.18 & 0.18 & 0.19 & 0.48 & 0.33 & 0.82 & 0.52 & 0.82 & 0.25 & 0.27 & 0.25 & 0.22 & 0.31 & 0.35 & 0.24 & 0.22 & 0.21 & 0.22 & 0.18 & 0.24 & 0.18 & 0.18 & 0.19 \\
\hline-0.20 & 0.16 & 0.20 & 0.16 & 0.16 & 0.16 & 0.25 & 0.25 & 0.82 & 0.29 & 0.33 & 0.18 & 0.20 & 0.16 & 0.16 & 0.17 & 0.34 & 0.20 & 0.18 & 0.16 & 0.17 & 0.16 & 0.20 & 0.16 & 0.16 & 0.16 \\
\hline-0.15 & 0.08 & 0.16 & 0.08 & 0.08 & 0.11 & 0.16 & 0.20 & 0.19 & 0.18 & 0.21 & 0.11 & 0.12 & 0.11 & 0.10 & 0.10 & 0.22 & 0.13 & 0.16 & 0.12 & 0.16 & 0.08 & 0.12 & 0.08 & 0.08 & 0.10 \\
\hline-0.10 & 0.04 & 0.08 & 0.04 & 0.04 & 0.05 & 0.09 & 0.08 & 0.09 & 0.09 & 0.09 & 0.07 & 0.05 & 0.06 & 0.06 & 0.06 & 0.18 & 0.06 & 0.10 & 0.06 & 0.10 & 0.04 & 0.05 & 0.04 & 0.04 & 0.05 \\
\hline-0.05 & 0.02 & 0.02 & 0.02 & 0.02 & 0.02 & 0.02 & 0.02 & 0.01 & 0.01 & 0.01 & 0.02 & 0.02 & 0.02 & 0.02 & 0.01 & 0.02 & 0.02 & 0.04 & 0.02 & 0.02 & 0.02 & 0.02 & 0.01 & 0.01 & 0.01 \\
\hline 0 & 0 & 0 & 0 & 0 & 0 & 0 & 0 & 0 & 0 & 0 & 0 & 0 & 0 & 0 & 0 & 0 & 0 & 0 & 0 & 0 & 0 & 0 & 0 & 0 & 0 \\
\hline 0.05 & 0.03 & 0.02 & 0.03 & 0.02 & 0.03 & 0.11 & 0.02 & 0.13 & 0.10 & 0.08 & 0.09 & 0.02 & 0.06 & 0.05 & 0.04 & 0.37 & 0.01 & 0.04 & 0.03 & 0.03 & 0.03 & 0.01 & 0.03 & 0.02 & 0.03 \\
\hline 0.10 & 0.06 & 0.05 & 0.06 & 0.05 & 0.06 & 0.21 & 0.05 & 0.31 & 0.18 & 0.12 & 0.12 & 0.05 & 0.12 & 0.10 & 0.12 & 0.75 & 0.03 & 0.07 & 0.05 & 0.06 & 0.06 & 0.03 & 0.06 & 0.05 & 0.06 \\
\hline 0.15 & 0.08 & 0.10 & 0.08 & 0.08 & 0.07 & 0.26 & 0.18 & 0.31 & 0.28 & 0.12 & 0.17 & 0.16 & 0.17 & 0.15 & 0.17 & & 0.10 & 0.09 & 0.08 & 0.08 & 0.08 & 0.10 & 0.08 & 0.08 & 0.07 \\
\hline 0.20 & 0.11 & 0.16 & 0.11 & 0.11 & 0.11 & 0.30 & 0.31 & 0.35 & 0.33 & 0.19 & 0.22 & 0.21 & 0.21 & 0.18 & 0.21 & & 0.16 & 0.14 & 0.14 & 0.11 & 0.11 & 0.16 & 0.11 & 0.11 & 0.11 \\
\hline 0.25 & 0.17 & 0.18 & 0.17 & 0.17 & 0.14 & 0.31 & 0.34 & 0.36 & 0.35 & 0.21 & 0.25 & 0.25 & 0.24 & 0.22 & 0.24 & & 0.16 & 0.15 & 0.14 & 0.12 & 0.17 & 0.16 & 0.15 & 0.14 & 0.12 \\
\hline 0.30 & 0.17 & 0.18 & 0.17 & 0.17 & 0.14 & 0.31 & 0.34 & 0.36 & 0.35 & 0.21 & 0.25 & 0.25 & 0.24 & 0.22 & 0.24 & & 0.16 & 0.15 & 0.14 & 0.12 & 0.17 & 0.16 & 0.15 & 0.14 & 0.12 \\
\hline 0.35 & 0.20 & 0.29 & 0.20 & 0.20 & 0.17 & 0.31 & 0.34 & 0.75 & 0.35 & 0.21 & 0.26 & 0.33 & 0.28 & 0.25 & 0.30 & & 0.18 & 0.16 & 0.15 & 0.14 & 0.20 & 0.18 & 0.16 & 0.15 & 0.14 \\
\hline 0.40 & 0.25 & 0.34 & 0.25 & 0.25 & 0.25 & 0.33 & 0.35 & & 0.35 & 0.33 & 0.30 & 0.37 & 0.31 & 0.29 & 0.31 & & 0.19 & 0.18 & 0.16 & 0.15 & 0.25 & 0.19 & 0.18 & 0.16 & 0.15 \\
\hline 0.45 & 0.25 & 0.35 & 0.25 & 0.25 & 0.25 & 0.35 & 0.35 & & 0.51 & 0.33 & 0.31 & 0.43 & 0.31 & 0.30 & 0.31 & & 0.19 & 0.19 & 0.18 & 0.16 & 0.25 & 0.19 & 0.19 & 0.18 & 0.16 \\
\hline 0.50 & 0.28 & 0.53 & 0.28 & 0.28 & 0.28 & 0.36 & 0.42 & & 0.51 & 0.36 & 0.31 & 0.43 & 0.31 & 0.31 & 0.31 & & 0.20 & 0.20 & 0.19 & 0.18 & 0.28 & 0.20 & 0.20 & 0.19 & 0.18 \\
\hline 0.55 & 0.28 & 0.54 & 0.28 & 0.28 & 0.28 & 0.75 & 0.52 & & 0.56 & 0.36 & 0.31 & 0.49 & 0.31 & 0.31 & 0.31 & & 0.24 & 0.22 & 0.21 & 0.19 & 0.28 & 0.24 & 0.22 & 0.21 & 0.19 \\
\hline 0.60 & 0.30 & 0.75 & 0.30 & 0.30 & 0.28 & & 0.52 & & 0.56 & 0.75 & 0.31 & 0.75 & 0.35 & 0.34 & 0.36 & & 0.34 & 0.25 & 0.25 & 0.22 & 0.30 & 0.34 & 0.25 & 0.25 & 0.22 \\
\hline 0.65 & 0.31 & & 0.31 & 0.31 & 0.31 & & 0.75 & & 0.56 & & 0.33 & & 0.36 & 0.35 & 0.75 & & 0.34 & 0.26 & 0.26 & 0.22 & 0.31 & 0.34 & 0.26 & 0.26 & 0.22 \\
\hline 0.70 & 0.31 & & 0.31 & 0.31 & 0.31 & & & & 0.56 & & 0.33 & & 0.75 & 0.35 & & & & 0.27 & 0.27 & 0.23 & 0.31 & & 0.27 & 0.27 & 0.23 \\
\hline 0.75 & 0.31 & & 0.31 & 0.31 & 0.31 & & & & 0.75 & & 0.35 & & & 0.35 & & & & 0.30 & 0.27 & 0.26 & 0.31 & & 0.30 & 0.27 & 0.26 \\
\hline 0.80 & 0.31 & & 0.31 & 0.31 & 0.31 & & & & & & 0.36 & & & 0.35 & & & & 0.30 & 0.30 & 0.26 & 0.31 & & 0.30 & 0.30 & 0.26 \\
\hline 0.85 & 0.31 & & 0.31 & 0.31 & 0.31 & & & & & & 0.75 & & & 0.35 & & & & 0.34 & 0.31 & 0.27 & 0.31 & & 0.31 & 0.31 & 0.27 \\
\hline 0.90 & 0.31 & & 0.31 & 0.31 & 0.31 & & & & & & & & & 0.75 & & & & 0.34 & 0.34 & 0.27 & 0.31 & & 0.31 & 0.31 & 0.27 \\
\hline 0.95 & & & & & 0.33 & & & & & & & & & & & & & 0.35 & 0.34 & 0.30 & & & 0.35 & 0.34 & 0.30 \\
\hline
\end{tabular}

For brevity, equivalent information for RSA-SRSS (error 2) and RSA-CQC (error 3), is given in the Supplementary Materials: Figure S10 (error 2, IPS5); Figure S11 (error 2, $I_{T L L 1}$ ); 
Figure S12 (error 2, $I_{A 1}$ ); Figure S13 (error 3, $\left.I_{P S 5}\right)$; Figure S14 (error 3, $I_{T L L 1}$ ); Figure S15 (error $3, I_{A 1}$ ); Table $S 1$ (error 2, $\left.I_{P S 5}\right)$; Table S2 (error 2, $I_{T L L 1}$ ); Table S3 (error 2, $I_{A 1}$ ); Table S4 (error 3, $I_{P S 5}$ ); Table S5 (error 3, $I_{T L L 1}$ ); Table S6 (error 3, $\left.I_{A 1}\right)$. For these procedures, the relationship between max and min error and the four selected NPIs is kept, but it is not always feasible to control the error for all variables; as an example, focusing on $I_{P S 5}$ and RSA-SRSS (Figure S10) it is only possible to keep the conservative error (max) for $a_{2}$ or $V_{\mathrm{D} 2}$ at central story or roof below a reasonable threshold if the value of $I_{P S 5}$ is practically 0; this trend is consistent for the four selected NPIs. Even so, limitation of the indices is still valuable to keep other relevant variables under control.

\section{Conclusions}

Addition of linear viscous dampers to frame structures generally results in nonproportional damping, characterized by a populated modal damping matrix and complexvalued damped modes. A well-established procedure to obtain an approximate response of such systems is to apply classical modal analysis neglecting the off-diagonal terms of the modal damping matrix. This study focuses on measurement of the errors incurred with this approximation, their relationship with parameters describing the structural system, and their possible correlation with non-proportionality indices, originally proposed in the literature to measure the extent of damping non-proportionality. A numerical study is performed, including a wide variety of representative frame-type and shear-type planar structures, added damping systems, and amounts of added and inherent damping, subjected to collections of scaled accelerograms. The errors between the 'exact' numerical response and the approximate responses obtained by two procedures based in modal analysis are considered. The two procedures are Time History Modal Analysis (THMA) and Response Spectrum Method (RSA), and in the latter two different combination rules, square-root-of-sum-of-squares (RSA-SRSS) and complete quadratic combination (RSACQC) are included. Negative errors indicate that the approximate method under-estimates the results; conversely, positive errors indicate result over-estimation. Based on results of this study, the following conclusions are drawn:

(1) The error in THMA is only mildly dependent on inherent damping and ground type; its median value is close to 0 and generally $80 \%$ of the results are within a range of $[-0.15 ;+0.25]$; conservative $(\max )$ errors are larger than un-conservative ( $\mathrm{min}$ ) errors. Large error dispersion occurs at the bottom and top of the frames, with a negative correlation between both stories. Shear-type systems present, in general, larger extreme errors than frame-type systems.

(2) RSA present similar trends to THMA, but with larger dispersion and a tendency to unconservative errors at first story and over-conservative errors at the roof. RSA-CQC offers a smaller dispersion than RSA-SRSS.

(3) To keep the error under a certain threshold in THMA and RSA, simple criteria based on frame and damping system properties cannot be proposed from the data available, as parameters that are significant for the response averaged through stories, central story, first story and roof are generally different. Moreover, values of parameters that reduce the response error in one story might trigger a large error in another.

(4) A correlation of the max and min error in THMA and RSA with four existing nonproportionality indices has been empirically proved and can be used as an alternative method to keep the error under control, especially for THMA. Tabulated data for different error thresholds are provided.

The tabulated data can be used to assess the adequacy of modal analysis in buildings equipped with supplemental damping. The underlying concepts can be extended to other engineering problems such as buildings with base isolation, or placement of viscous dampers for vibration control in high-speed railway bridges, etc.

These conclusions are limited to linear systems with added linear viscous dampers within the range of structural parameters defined in the parametric study, where only height-wise decreasing stiffness and damping configurations have been considered. How- 
ever, the range of parameters is wide enough to include most realistic systems. The study included only far-field motions recorded on firm soil conditions. For site classes B and C no significant differences in error results were found. The flexibility of auxiliary elements in the damping systems was not included. The analysis included all modes with exact spectral response, so the influence of these two sources of error (mode truncation and approximate spectral response) was not considered.

Supplementary Materials: The following are available online at https:/ /www.mdpi.com/article/10 .3390 /app11136064/s1, Figure S1: Plots of RSA-SRSS errors (error 2), Figure S2: Plots of RSA-CQC errors (error 3), Figure S3: Bar plot for relative displacement $u$ for RSA-SRSS error (error 2) and RSA-CQC error (error 3), average of stories, first story and roof, Figure S4: Bar plot for relative velocity $v$ for RSA-SRSS error (error 2) and RSA-CQC error (error 3), average of stories, first story and roof, Figure S5: Bar plot for drift $\Delta$ for RSA-SRSS error (error 2) and RSA-CQC error (error 3), average of stories, first story and roof, Figure S6: Bar plot for drift velocity $\nabla$ for RSA-SRSS error (error 2) and RSA-CQC error (error 3), average of stories, first story and roof, Figure S7: Bar plot for acceleration $a$ for RSA-SRSS error (error 2) and RSA-CQC error (error 3), average of stories, first story and roof, Figure S8: Bar plot for story shear vs. for RSA-SRSS error (error 2) and RSA-CQC error (error 3), average of stories, first story and roof, Figure S9: Bar plot for damper shear $V_{\mathrm{D}}$ for RSA-SRSS error (error 2) and RSA-CQC error (error 3), average of stories, first story and roof, Figure S10: RSA-SRSS error (type 2) max and min envelope for all cases versus index $I_{P S 5}$, Figure S11: RSASRSS error (type 2) max and min envelope for all cases versus index $I_{T L L 1}$. Figure S12: RSA-SRSS error (type 2) max and min envelope for all cases versus index $I_{A 1}$, Figure S13: RSA-CQC error (type 3) max and min envelope for all cases versus index $I_{P S 5}$, Figure S14: RSA-CQC error (type 3) max and min envelope for all cases versus index $I_{T L L 1}$, Figure S15: RSA-CQC error (type 3) max and min envelope for all cases versus index $I_{A 1}$. Table S1: Maximum value of $I_{P S 5}$ to limit the max and min RSA-SRSS error (error 2), Table S2: Maximum value of $I_{T L L 1}$ to limit the max and min RSA-SRSS error (error 2), Table S3: Maximum value of $I_{A 1}$ to limit the max and min RSA-SRSS error (error 2), Table S4: Maximum value of $I_{P S 5}$ to limit the max and min RSA-CQC error (error 3), Table S5: Maximum value of $I_{T L L 1}$ to limit the max and min RSA-CQC error (error 3), Table S6: Maximum value of $I_{A 1}$ to limit the max and min RSA-CQC error (error 3).

Author Contributions: Conceptualization, methodology and validation J.C. and A.B.; software, J.C.; writing —original draft preparation, J.C.; writing—review and editing, J.C. and A.B. All authors have read and agreed to the published version of the manuscript.

Funding: This research received no external funding.

Institutional Review Board Statement: Not applicable.

Informed Consent Statement: Not applicable.

Data Availability Statement: Data available from authors on demand.

Conflicts of Interest: The authors declare no conflict of interest.

\section{Appendix A. Description of Non-Proportionality Indices}

This appendix contains the analytical description of non-proportionality indices. Details of each index can be found in the original papers referenced, but a brief analytical description using a unified notation is provided for completeness. When an index is modedependent, its global version is calculated as the average across modes. Some additional concepts related to the free vibration solution of damped systems must be introduced prior to the description of the indices. The general equation of motion described by Equation (1) can be written as $[10,11,26]$ :

$$
[\mathbf{a}][\dot{\mathbf{z}}]+[\mathbf{b}][\mathbf{z}]=[\mathbf{p}],
$$

where:

$$
[\mathbf{a}]=\left[\begin{array}{cc}
\mathbf{c} & \mathbf{m} \\
\mathbf{m} & 0
\end{array}\right] ;[\mathbf{z}]=\left[\begin{array}{c}
\mathbf{u} \\
\dot{\mathbf{u}}
\end{array}\right] ;[\mathbf{b}]=\left[\begin{array}{cc}
\mathbf{k} & 0 \\
0 & -\mathbf{m}
\end{array}\right] ;[\mathbf{p}]=\left[\begin{array}{c}
-\mathbf{m} \mathbf{\imath} a_{g}(t) \\
0
\end{array}\right] .
$$


[x] indicates an extended matrix (a matrix with matrix components), and $\mathbf{0}$ is a null matrix of the required dimensions and units. The eigenproblem stated by the homogeneous counterpart of Equation (A1) leads to $2 \mathrm{~N}$ eigenvectors and eigenvalues that, for undercritically damped systems, can be expressed as:

$$
\begin{gathered}
{\left[\boldsymbol{\Psi}_{E}\right]=\left[\begin{array}{cc}
\boldsymbol{\Psi} & -\mathbf{\Psi} \\
\mathbf{\Psi} \boldsymbol{\Lambda} & \mathbf{\Psi} \boldsymbol{\Lambda}
\end{array}\right]} \\
{\left[\boldsymbol{\Lambda}_{E}\right]=\left[\begin{array}{cc}
\boldsymbol{\Lambda} & \frac{0}{} \\
0 & \boldsymbol{\Lambda}
\end{array}\right],}
\end{gathered}
$$

where $\overline{\mathbf{x}}$ indicates the complex conjugate of $\mathbf{x}$, and $\boldsymbol{\Psi}=\left[\psi_{1}, \ldots, \psi_{N}\right]$ with $\psi_{j}=\left[\psi_{1 j}, \ldots, \psi_{N j}\right]^{\mathrm{T}}$, where every term $\psi_{n j}$ is a complex number composed of real $\left(\psi_{R, n j}\right)$ and imaginary $\left(\psi_{I, n j}\right)$ parts. Likewise, $\Lambda$ is a diagonal matrix with $j^{\text {th }}$ component $\lambda_{j}=-\xi_{j} \omega_{j}+i \omega_{j}\left(1-\xi_{j}^{2}\right)^{0.5}$, where $i=\sqrt{-1}$. $\xi_{j}$. and $\omega_{j}$ were already introduced in Section 2.1. $\left[\mathbf{\Psi}_{E}\right]$ diagonalizes both [a] and [b]. Thus, the damped modes can be normalized in a similar way to the undamped modes by solving $\left[\hat{\mathbf{Y}}_{E}\right]^{\mathrm{T}}[\mathbf{a}]\left[\hat{\mathbf{Y}}_{E}\right]=[\mathbf{I}]$, leading to the normalized modal matrix $\left[\hat{\mathbf{\Psi}}_{E}\right]=\left[\boldsymbol{\Psi}_{E}\right][\mathbf{A}]^{-1 / 2}$, where $[\mathbf{A}]=\left[\mathbf{\Psi}_{E}\right]^{\mathrm{T}}[\mathbf{a}]\left[\mathbf{\Psi}_{E}\right]$ is a diagonal matrix.

Appendix A.1. Warburton and Soni

Warburton and Soni [28] compared the resonant response for a MDOF with nonclassical damping excited harmonically in the $n^{\text {th }} \mathrm{DOF}$, and its approximate solution obtained neglecting off-diagonal terms, deriving the following condition:

$$
\left|\hat{\varphi}_{n j}\right| \gg \max _{1 \leq s \leq N, s \neq j}\left(\left|\frac{\hat{C}_{j s} \omega_{j} \hat{\varphi}_{n s}}{\omega_{s}^{2}-\omega_{j}^{2}}\right|\right),
$$

to be fulfilled at every DOF $n$, whereupon the following index can be defined for mode $j$ :

$$
I_{W S 1, j}=\max _{1 \leq n \leq N}\left(\frac{\max _{1 \leq s \leq N, s \neq j}\left(\left|\hat{C}_{j s} \omega_{j} \hat{\varphi}_{n s} /\left(\omega_{s}^{2}-\omega_{j}^{2}\right)\right|\right)}{\left|\hat{\varphi}_{n j}\right|}\right) .
$$

This index was not presented in this form by Warburton and Soni, but it has been derived modifying an expression in the original referenced paper. This index, besides not being normal, is quite sensitive to small values of modal displacements which might happen close to nodal points in higher modes; additionally, it is not formally proved that an index derived for harmonic motion might be reasonably exact for random excitation. Assuming that the order of magnitudes of modal displacements is similar, the authors rewrite Equation (A5) to formulate the following condition:

$$
\xi_{j}<\varepsilon \min _{1 \leq s \leq N, s \neq j}\left(\left|\frac{\hat{C}_{j j}}{2 \hat{C}_{j s}}\left(\frac{\omega_{s}^{2}}{\omega_{j}^{2}}-1\right)\right|\right),
$$

where $\varepsilon$ is an error parameter that should be close to 0 , and therefore can be assimilated to an index, albeit not a normal one:

$$
I_{W S 2, j}=\frac{\xi_{j}}{\min _{1 \leq s \leq N, s \neq j}\left(\left|\frac{\hat{C}_{j j}}{2 \hat{C}_{j s}}\left(\frac{\omega_{s}^{2}}{\omega_{j}^{2}}-1\right)\right|\right)} .
$$




\section{Appendix A.2. Prater and Singh}

Prater and Singh [29] proposed several indices. After normalization of the modal vectors to the maximum of each mode (so that the modal displacements magnitudes are no larger than 1), the area of the polygon formed by the complex plane representation of the modal vectors for a given mode $j, A_{1, j}$, is taken as representative of the phase differences between modes. This area is compared with the maximum possible area $A_{1, \max }$ corresponding to a regular polygon inscribed in a circle of unit radius, given by $N \cos (\pi / N) \sin (\pi / N)$. Thus:

$$
I_{P S 1, j}=\frac{A_{1, j}}{A_{1, \max }} .
$$

As discussed by Bhaskar [33], the polygon area is ambiguous in the sense that it is dependent on the order of DOFs, but this difficulty is avoided considering only the convex polygon. Another difficulty is that this index is not defined for systems with only $2 \mathrm{DOFs}$; this could be overridden by adding the point $(0,0)$ to the set, giving rise to an alternative index, not discussed here (in this study $N>2$ for all systems).

Higher modes will always present a higher index because the presence of nodal points ensures that points representing modal vectors will lie in different quadrants of the complex plane. To override this problem, modal vectors in the 2 nd or 3 rd quadrant can be rotated $180^{\circ}$, so that all polygons lie in the 1st and 4th quadrant. These are referred to normalized-rotated modal vectors. The polygon area in mode $j$ is referred to as $A_{2, j}$, and the maximum area $A_{2, \max }$ is given by $(N-1) \cos (\pi / 2(N-1)) \sin (\pi /(2 N-1))$, that is half the area of a regular polygon of $(2 \mathrm{~N}-2)$ sides inscribed in a circle of unit radius; the index reads:

$$
I_{P S 2, j}=\frac{A_{2, j}}{A_{2, \max }},
$$

where the area of the convex polygon should be considered, and the index is again undefined for 2 DOF systems.

Additionally, considering the phase of the normalized-rotated complex modes, $\theta_{n j}=\arg \left(\varphi_{n j}\right)$ (where $\arg (\cdot)$ indicates the argument function), these authors define the simple index:

$$
I_{P S 3, j}=\frac{\sum_{n=1}^{N} 2\left|\theta_{n j}\right|}{N \pi},
$$

normalized assuming the phase angle lies between $-\pi / 2$ and $\pi / 2$. This normal index is not independent of mode rotations; for instance, if two DOFs present the same maximum modal coordinate the value of the index will depend upon which one is chosen to normalize. To avoid this problem, a second, more meaningful normal index is given by:

$$
I_{P S 4, j}=\frac{\max \left(\left|\theta_{n j}\right|\right)-\min \left(\left|\theta_{n j}\right|\right)}{\pi},
$$

corresponding to the normalized angle spanned by the normalized-rotated modal vectors.

Finally, two indices are derived based on the normalized modal damping matrix $\hat{\mathbf{C}}$; the relative magnitude of the non-diagonal terms can be used as a measure of the degree of non-proportionality:

$$
I_{P S 5}=\frac{\sum_{j=1}^{N} \sum_{n=1, n \neq j}^{N}\left|\hat{C}_{n j}\right|}{\sum_{j=1}^{N} \sum_{n=1}^{N}\left|\hat{C}_{n j}\right|},
$$

a normal index which is simple to compute; a mode-dependent index can be calculated ignoring the first summation sign in the numerator and denominator:

$$
I_{P S 5, j}=\frac{\sum_{n=1, n \neq j}^{N}\left|\hat{C}_{n j}\right|}{\sum_{n=1}^{N}\left|\hat{C}_{n j}\right|} .
$$


In this case the global value obtained by Equation (A13) is not coincident with the average of the modal cases obtained by Equation (A14) $I_{P S 5, a v}$. An optional way to measure the importance of off-diagonal terms is given by the matrix determinant. If $\hat{\mathbf{C}}_{o}$ is the matrix formed suppressing the diagonal terms in $\hat{\mathbf{C}}$,

$$
I_{P S 6}=\frac{\operatorname{det}\left(\hat{\mathbf{C}}_{\mathbf{o}}\right)}{\operatorname{det}(\hat{\mathbf{C}})}
$$

a normal index that cannot be applied to single modes. A third index is proposed by these authors based on the response of the system to harmonic motion. Because it involves full evaluation of the system's response, it is not a practical option for design purposes and is left out of this study.

Appendix A.3. Nair and Singh

Nair and Singh [38] proposed an index based on Equation (5):

$$
\begin{gathered}
\mathbf{E}=\mathbf{c m}^{-1} \mathbf{k}-\mathbf{k m}^{-1} \mathbf{c}, \\
I_{N S 1}=\sum_{n=1}^{N} \sum_{j=1}^{N}\left|E_{n j}\right| .
\end{gathered}
$$

For systems with proportional damping, this function equals 0 . However, it presents several problems: first, the function is not upper-bounded; second, the function has units ([MASS][TIME $\left.\left.{ }^{-3}\right]\right)$. Thus, it cannot be considered a proper index. Furthermore, it requires explicit knowledge of the stiffness matrix $\mathbf{k}$ (although in practical situations, it can be derived from mass matrix $\mathbf{m}$ and modal properties as $\mathbf{k}=\mathbf{m} \boldsymbol{\Phi} \Omega^{2} \boldsymbol{\Phi}^{-1}$ ).

\section{Appendix A.4. Claret and Venancio-Filho}

These authors [30] proposed an index based on comparison of off-diagonal and diagonal terms of the normalized modal damping matrix $\hat{\mathbf{C}}$ :

$$
I_{C V}=\max \left[\frac{\hat{C}_{n j}^{2}}{\hat{C}_{n n} \hat{C}_{j j}}\right]_{n \neq j} .
$$

The index is normal, but undefined for the (theoretical) purely undamped case.

\section{Appendix A.5. Tong, Liang and Lee}

The index proposed by these authors [31] relies in the normalized modal damping matrix $\hat{\mathbf{C}}$. The index is presented as:

$$
I_{T L L 1}=\frac{\hat{\sigma}_{\max }-\hat{\sigma}_{\min }}{\hat{\sigma}_{\max }+\hat{\sigma}_{\min }}
$$

where $\hat{\sigma}_{\max }$ and $\hat{\sigma}_{\max }$ are the maximum and minimum eigenvalues of $\hat{\mathbf{C}}_{d}^{-1} \hat{\mathbf{C}}$. Because of the properties of the normalized modal damping matrix, the index is normal. Moreover, the authors discuss three important properties of the index, namely its monotonic increase with the non-diagonal terms of $\hat{\mathbf{C}}$, its independency from $\hat{\mathbf{C}}$ being scaled, and its convergence to 0 for proportional damping.

\section{Appendix A.6. Bhaskar}

This author proposed three different indices [32,33]. The first index is again based on the dominance of off-diagonal terms in the modal matrix:

$$
I_{B 1}=\max _{1 \leq k \leq N}\left(\sum_{j=1, j \neq k}^{N} \frac{\left|\hat{C}_{k j}\right|}{\left|\hat{C}_{k k}\right|}\right) .
$$


The second index is based on the concept that complex representation of modal vectors for a proportionally damped system will radiate from the origin. For a generally damped structure and a given mode $j$, a certain rotation $\theta_{j}$ of the coordinate system can be found to minimize the sum of the squares of the ordinates of the modal displacements; in a proportionally damped system, this would be the natural orientation of modal vectors; deviation of each modal displacement from this orientation is indicative of non-proportionality. The expression of the modal vector $\psi^{\prime}{ }_{n j}$ in the rotated coordinate system is:

$$
\psi_{n j}^{\prime}=x \prime_{n j}+i y \prime_{n j}=\left|\psi_{n j}\right| \exp \left(\left(\theta_{n j}-\theta_{j}\right) i\right),
$$

where $\psi^{\prime}{ }_{n j}$ is the $n^{\text {th }}$ component of the $j^{\text {th }}$ modal vector expressed in the new coordinate system, $x \prime_{n j}$ its real component, $y{ }_{n j}$ its imaginary component, $\exp (\cdot)$ the exponential function and $|\cdot|$ the modulus function. The sum of squared abscissas and ordinates is, respectively:

$$
\begin{aligned}
& X \prime_{j}=\sum_{n=1}^{N}\left(x_{n j}\right)^{2}, \\
& Y \prime_{j}=\sum_{n=1}^{N}\left(y^{\prime}{ }_{n j}\right)^{2} .
\end{aligned}
$$

Minimization of $Y \prime_{j}$ (or maximization of $X \prime_{j}$ ), will render $\theta_{j}$ (a closed-form solution is given by this author, omitted here for brevity). Then, the index is expressed as:

$$
I_{B 2, j}=\sqrt{Y \prime_{j} / X \prime_{j}}
$$

The third index proposed by this author is derived obtaining the area of the triangle formed by the modal displacement vector and its projection on the $x /$ axis. This area is maximal when the projection is $\left|\psi_{n j}\right| \sqrt{2}$; therefore:

$$
I_{B 3, j}=\sum_{n=1}^{N}\left(x \prime_{j} y \prime_{j}\right) /\left(2 A \prime_{j, \max }\right),
$$

where:

$$
A \prime_{j, \max }=\sum_{n=1}^{N}\left|\psi_{n j}\right|^{2} / 4
$$

$I_{B 2}$ and $I_{B 3}$ are normal; they are also invariant to scaling of the modal vectors. Notice that for evaluation of $I_{B 2, j}$ and $I_{B 3, j}$ there is no need to use normalized modes.

\section{Appendix A.7. Liu, Kujath and Zheng}

These authors proposed six different indices [34,35]; three of them are based on modal displacements and three on properties of the modal damping matrix. To obtain the former three indices, it is first necessary to manipulate the modes with a procedure similar to the one proposed by Bhaskar. The modal vector of mode $j, \boldsymbol{\Psi}_{j}$ is normalized to its maximum component $\Psi_{j, \max }$, and rotated counterclockwise by $45^{\circ}$ :

$$
\widetilde{\mathbf{\Psi}}_{j}=\frac{\mathbf{\Psi}_{j}}{\boldsymbol{\Psi}_{j, \max }} \exp \left(\frac{i \pi}{4}\right)
$$

the first index proposed by the authors is based on the increasing correlation (due to modal displacement alignment) between imaginary and real parts of $\widetilde{\Psi}_{j}$ as proportionality increases. Thus:

$$
I_{L K Z 1, j}=1-\frac{\left|\widetilde{\mathbf{\Psi}}_{R, j}^{\mathrm{T}} \tilde{\mathbf{\Psi}}_{\mathrm{I}, \mathrm{j}}\right|}{\sqrt{\left[\tilde{\mathbf{\Psi}}_{R, j}^{\mathrm{T}} \tilde{\mathbf{\Psi}}_{\mathrm{R}, \mathrm{j}}\right]\left[\tilde{\mathbf{\Psi}}_{I, j}^{\mathrm{T}} \tilde{\mathbf{\Psi}}_{\mathrm{I}, \mathrm{j}}\right]}}
$$


where the second part of the right term measures the correlation between real and imaginary parts of the modal vector.

The best fit angle of the rotated displacements is found with a least square method:

$$
\theta_{j}=\arctan \left(\frac{\widetilde{\mathbf{\Psi}}_{R, j}^{\mathrm{T}} \widetilde{\Psi}_{I, j}}{\widetilde{\mathbf{\Psi}}_{R, j}^{\mathrm{T}} \widetilde{\Psi}_{R, j}}\right),
$$

where $\arctan (\cdot)$ is the arctangent function, $\tilde{\mathbf{\Psi}}_{\mathrm{R}, j}$ is the real part of $\tilde{\mathbf{\Psi}}_{j}$ and $\tilde{\mathbf{\Psi}}_{\mathrm{I}, j}$ is its imaginary part. Modal vectors are then rotated clockwise by the best fit angle:

$$
\overbrace{\mathbf{\Psi}}=\widetilde{\Psi}_{j} \exp \left(-i \theta_{j}\right) .
$$

The imaginary part of $\overbrace{\Psi}$ is minimal (in the least square sense). The second index is simply a measure of the scatter of the imaginary parts of $\overbrace{\boldsymbol{\Psi}}$ :

$$
I_{L K Z 2, j}=\frac{\sum_{n=1}^{N}|\overbrace{\Psi_{I, n j}}|}{N} .
$$

To find the third index, the normalized modal matrix is first decomposed as:

$$
\overbrace{\mathbf{\Psi}}=\overbrace{\mathbf{\Psi}} \overbrace{\mathrm{T}}^{\mathbf{T}}=\overbrace{\mathbf{\Psi}}^{\mathrm{R}}\left(\mathbf{I}+i_{\mathrm{T}_{\mathrm{I}}}\right) \text {, }
$$

where $\overbrace{\Psi_{R}}$ is the real part of $\overbrace{\Psi}, \overbrace{\mathbf{T}}$ is complex with real part $\overbrace{\mathbf{T}}=\mathbf{I}$, and imaginary part $\overbrace{\mathbf{T}}{ }_{I}=\overbrace{\Psi_{R}}^{-1} \overbrace{\Psi_{I}}$. The magnitude of $\overbrace{T_{I}}$ is linked to modal coupling and therefore the following index is proposed:

$$
I_{L K Z 3, j}=\frac{\sum_{n=1}^{N}|\overbrace{T_{I, n j}}|}{N} .
$$

The next three indices proposed by these authors are based on modal information. Using the expansion of the normalized modal matrix:

$$
\hat{\mathbf{\Psi}}=\hat{\mathbf{\Phi}} \hat{\mathbf{T}} \Rightarrow \hat{\mathbf{T}}=\hat{\boldsymbol{\Phi}}^{-1} \hat{\mathbf{Y}} .
$$

The fourth index is a counterpart of $I_{P S 5}$ based on $\hat{\mathbf{T}}$ instead of $\hat{\mathbf{C}}$ :

$$
I_{L K Z 4}=\frac{\sum_{j=1}^{N} \sum_{n=1, n \neq j}^{N}\left|\hat{T}_{n j}\right|}{\sum_{j=1}^{N} \sum_{n=1}^{N}\left|\hat{T}_{n j}\right|},
$$

it must be noticed that $I_{L K Z 4}$ is dependent from the normalization used for both the damped modes $\hat{\boldsymbol{\Psi}}$ and undamped modes $\hat{\boldsymbol{\Phi}}$. It can be verified that if the index is alternatively expressed as mode-dependent, see Equation (A14):

$$
I_{L K Z 4, j}=\frac{\sum_{n=1, n \neq j}^{N}\left|\hat{T}_{n j}\right|}{\sum_{n=1}^{N}\left|\hat{T}_{n j}\right|},
$$

it does not depend on the normalization of the damped modes. $I_{L K Z 4, a v}$ is thus defined as the average of $I_{L K Z 4, j}$. 
A counterpart index to $I_{T L L 1}$ is proposed as:

$$
I_{L K Z 5}=\frac{\bar{\sigma}_{\max }-\bar{\sigma}_{\min }}{\bar{\sigma}_{\max }+\bar{\sigma}_{\min }}
$$

where $\bar{\sigma}_{\max }$ and $\bar{\sigma}_{\min }$ are the maximum and minimum eigenvalues of the real-valued matrix $\mathbf{G}$ obtained as $\bar{G}_{n, j}=\left|G_{n, j}\right|$ from the absolute terms of the complex-valued matrix $\mathbf{G}=\hat{\mathbf{T}}_{d}^{-1} \hat{\mathbf{T}}$, where $\hat{\mathbf{T}}_{d}$ is the diagonal matrix formed suppressing off-diagonal terms in $\hat{\mathbf{T}}$.

Finally, following the same rationale as in $I_{L K Z 3}$, the sixth index is based on the off-diagonal elements of $\mathrm{G}$ :

$$
I_{L K Z 6, j}=\frac{\sum_{n=1, n \neq j}^{N}\left|\bar{G}_{n j}\right|}{N-1},
$$

Appendix A.8. Prells and Friswell

These authors [36] propose decomposition of $\hat{\Psi}$ in the following form:

$$
\hat{\mathbf{\Psi}}=\hat{\mathbf{\Psi}}_{\mathrm{R}}(\mathbf{I}-i \boldsymbol{\Theta}),
$$

where $\boldsymbol{\Theta}=-\hat{\mathbf{\Psi}}_{\mathrm{I}} \hat{\mathbf{\Psi}}_{\mathrm{R}}^{-1}$ can be shown to be an orthonormal matrix. An important result proved by these authors is that damping is proportional if, and only if, $\boldsymbol{\Theta}=\mathbf{I}$. Consequently, the degree of proportionality can be measured as:

$$
I_{P F 1}=\frac{\boldsymbol{\Theta}-\mathrm{I}}{2},
$$

where $\|\mathbf{x}\|$ indicates the spectral matrix norm of $\mathbf{x}$. Unfortunately, calculation of this index has been found to be impaired by numerical errors, as the condition number of $\hat{\mathbf{\Psi}}_{R}$ is, in many usual cases, high.

\section{Appendix A.9. Adhikari}

Scaling of complex modes by complex scalars significantly changes their geometric appearance; a certain scaling allows the damped modes to become as close as possible (again in the least square sense) to the undamped normal modes; Adhikari [37] referred to these as optimal complex modes, and derived an expression to find them; for mode $j$ :

$$
\breve{\mathbf{\Psi}}_{j}=\frac{\boldsymbol{\Psi}_{\mathrm{R}, j}\left(\mathbf{\Psi}_{\mathrm{R}, j}^{\mathrm{T}} \hat{\boldsymbol{\phi}}_{j}\right)+\mathbf{\Psi}_{\mathrm{I}, j}\left(\mathbf{\Psi}_{\mathrm{I}, j}^{\mathrm{T}} \hat{\boldsymbol{\phi}}_{j}\right)}{\left|\boldsymbol{\Psi}_{\mathrm{R}, j}\right|^{2}+\left|\mathbf{\Psi}_{\mathrm{I}, j}\right|^{2}}+i \frac{\mathbf{\Psi}_{\mathrm{I}, j}\left(\mathbf{\Psi}_{\mathrm{R}, j}^{\mathrm{T}} \hat{\boldsymbol{\phi}}_{j}\right)+\boldsymbol{\Psi}_{\mathrm{R}, j}\left(\mathbf{\Psi}_{\mathrm{I}, j}^{\mathrm{T}} \hat{\boldsymbol{\phi}}_{j}\right)}{\left|\boldsymbol{\Psi}_{\mathrm{R}, j}\right|^{2}+\left|\boldsymbol{\Psi}_{\mathrm{I}, j}\right|^{2}} .
$$

Because optimal modes are closest to natural modes, the distance between them is a direct measure of non-proportionality:

$$
I_{A 1, j}=\frac{\left|\breve{\mathbf{\Psi}}_{j}-\hat{\boldsymbol{\phi}}_{j}\right|}{\left|\hat{\boldsymbol{\phi}}_{j}\right|} .
$$

This author suggests the use of both mean and standard deviation across modes to characterize the overall non-proportionality of the system. The index is not sensitive to normalization of undamped modes in Equations (A41) and (A42), if there is consistency in the normalization used for both equations. Use of mass-normalized undamped modes in Equation (A41) results in optimal complex modes $\breve{\Psi}$ with units of [MASS ${ }^{-0.5}$ ].

\section{Appendix B. Determination of Range for Parameter $\lambda$}

$\lambda$ is defined in Section 2 as the ratio between the equivalent stiffness of beams $E I_{b, e q, n}$ and columns $E I_{c, e q, n}$ in the surrogate frames used in the study. This Appendix explains how 
a practical range of values for $\lambda$ (between 0.1 to 10 centered around 1 ) was determined. Figure Ala shows a regular steel frame, with identical span length $L$ at each bay and height $h$ at each story, where all the beam elements at story $n$ are similar and present a stiffness $E I_{b, n}$, where $E$ is the steel Young modulus and $I_{x, n}$ the relevant second moment of inertia of element $x$ at story $n$ for in-plane deformation. Likewise, all columns at story $n$ are assumed identical, with stiffness $E I_{\mathcal{C}, n}$. Shear and axial flexibility are neglected. The corresponding surrogate frame is shown in Figure A1b; the corresponding beam and column at level $n$ present stiffnesses $E I_{b, e q, n}$ and $E I_{c, e q, n}$, defined as

$$
\begin{gathered}
E I_{b, e q, n}=S E I_{b, n}, \\
E I_{c, e q, n}=(S+1) E I_{c, n} / 2,
\end{gathered}
$$

where $S$ is the number of bays of the original frame. This conversion assumes that the inflection points of all beams are located exactly at midspan, resulting in identical rotations for all joints at the same level; this is the same assumption used to transform regular frames to so-called fish-bone models [45], resulting in a stiffness matrix identical to that of the surrogate frame in this study. Typical moment-resisting frames are designed imposing capacity conditions at every joint in story $n$, such as:

$$
M_{\mathrm{R}, c, n}+M_{\mathrm{R}, c, n+1} \geq \gamma \sum M_{\mathrm{R}, b, n}
$$

where $M_{\mathrm{R}, c, n}$ and $M_{\mathrm{R}, c, n+1}$ represent the bending strength of columns at story $n$ and $n+1$, respectively, $\sum M_{R, b, n}$ is the sum of bending moment strength of beams concurring to the joint considered $\left(M_{\mathrm{R}, b, n}\right.$ at the edge, $2 M_{\mathrm{R}, b, n}$ at the inner joint, see Figure A1c), and $\gamma>1$ is a factor that in Eurocode 8 takes a value of 1.3 [42] (clause 4.4.2.3(4)); this condition must be applied at all joints for $n<N$. For the frame represented in Figure A1a, the condition imposed in the inner joint is more restrictive to columns. Two different limiting strength scenarios are assumed:

(1) $\quad M_{\mathrm{R}, c, n}=M_{\mathrm{R}, c, n+1}$ leading to the condition $M_{\mathrm{R}, c, n} \geq 1.3 M_{\mathrm{R}, b, n}$;

(2) $\quad M_{\mathrm{R}, c, n} \gg M_{\mathrm{R}, c, n+1}$ leading to the condition $M_{R, c, n} \geq 2.6 M_{\mathrm{R}, b, n}$.

Assuming IPE sections for beams and HEB sections for columns in the original frame, $E=210 \mathrm{GPa}$, and the same yield strength in both beams and columns, once the beam section is fixed, the values of $E I_{b, n}$ and $M_{\mathrm{R}, b, n}$ are known, and the flexural strength of the columns can be found according to strength scenario \#1 or \#2; the minimum HEB section providing this strength is determined and its stiffness $E I_{\mathcal{C}, n}$ obtained; then Equations (A43), (A44) and (7) are used to find values of $\lambda$ for different numbers of bays $S(1,2,3,4$, and 10$)$. The process is repeated for all possible IPE sections from IPE200 to IPE600. Relevant statistics (median $\mu$, standard deviation $\sigma$ and coefficient of variation $c_{v}$ ) of the results obtained for $\lambda$ across the full range of cross-sections are shown in Table A1; actual values are expected to lie between both strength scenarios; for the class of frames analyzed, $\lambda$ falls between 0.35 and 1.64 and $\lambda=1$ is adopted as central value. If the column yield strength adopted is 1.5 times the beam yield strength (for instance, for S235 beams and S355 columns), $\lambda$ lies between 0.58 and 2.75 . The range of values finally adopted is expanded considerably [between 0.1 and 10] and includes $\lambda=\infty$, corresponding to a pure shear frame.

It can be argued that, because the analysis performed in the parametric study is purely linear, imposition of strength conditions derived from ductility considerations is inadequate. However, it must be kept in mind that current codes impose similar design conditions for frames with or without added damping systems [8] (clause 18.2.1.1). Thus, although linear behavior is a reasonable design target for the former, capacity criteria and seismic details ensuring ductility must still be adopted, to consider the possibility of non-linear behavior, given the uncertainty in seismic input [3]. 


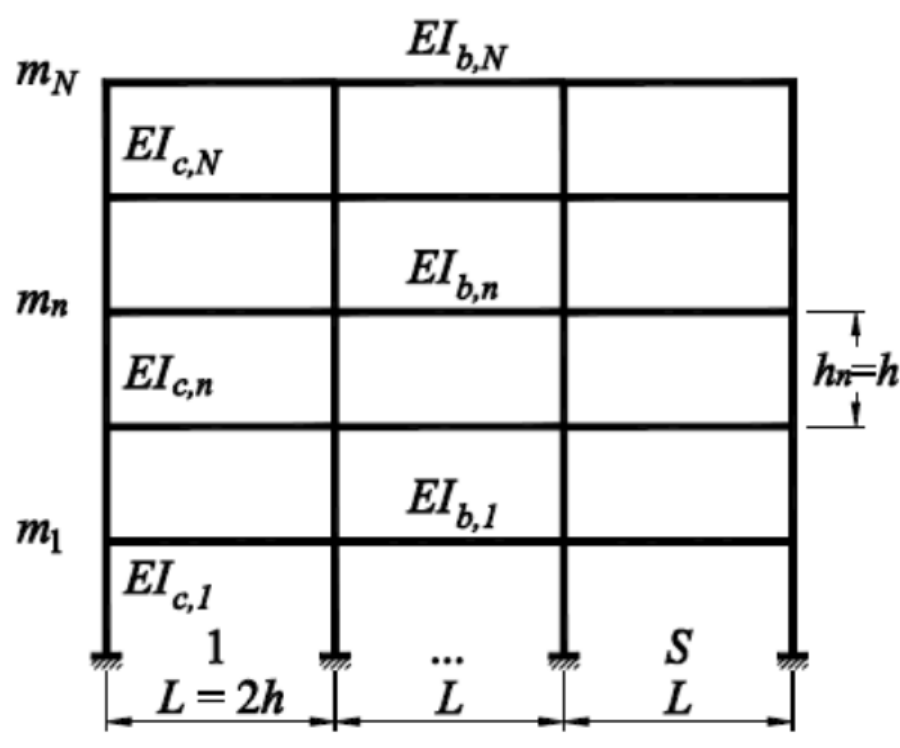

(a)

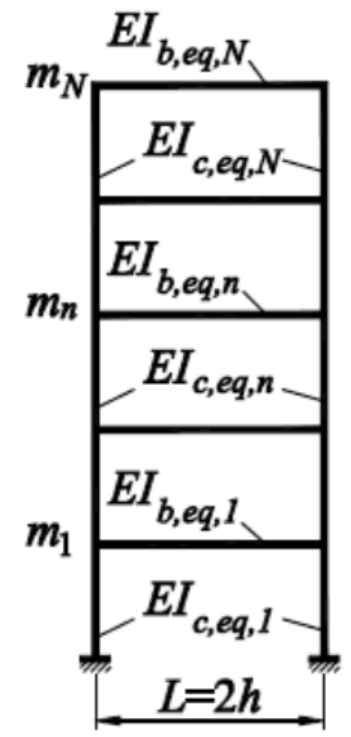

(b)
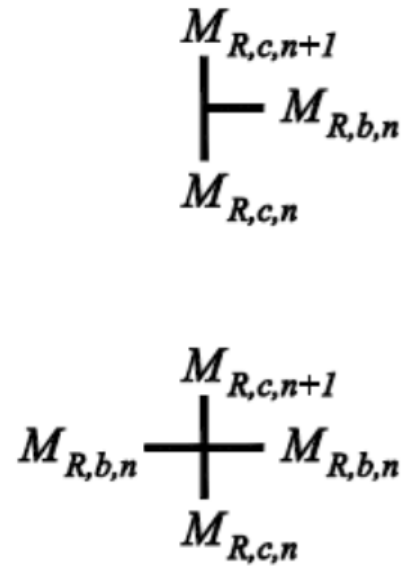

(c)

Figure A1. Original and surrogate frame: (a) original frame definition; (b) surrogate frame used in study; (c) capacity condition at joints.

Table A1. Summary statistics of $\lambda$ assuming IPE beams and HEB columns with equal yield strength, according to number of bays $S$ and relative column strength scenarios.

\begin{tabular}{|c|c|c|c|c|c|c|}
\hline \multirow[t]{2}{*}{$S$} & \multicolumn{3}{|c|}{$\begin{array}{c}\text { Scenario \#1 } \\
M_{\mathrm{R}, c, n}=M_{\mathrm{R}, c, n+1} \Rightarrow M_{\mathrm{R}, c, n} \geq 1.3 M_{\mathrm{R}, b, n}\end{array}$} & \multicolumn{3}{|c|}{$\begin{array}{c}\text { Scenario \#2 } \\
M_{\mathrm{R}, c, n}>>M_{\mathrm{R}, c, n+1} \Rightarrow M_{\mathrm{R}, c, n} \geq 2.6 M_{\mathrm{R}, b, n}\end{array}$} \\
\hline & $\mu$ & $\sigma$ & $c_{v}=\sigma / \mu$ & $\mu$ & $\sigma$ & $c_{v}=\sigma / \mu$ \\
\hline 1 & 0.90 & 0.11 & 0.12 & 0.35 & 0.05 & 0.14 \\
\hline 2 & 1.20 & 0.14 & 0.12 & 0.47 & 0.07 & 0.15 \\
\hline 3 & 1.35 & 0.16 & 0.12 & 0.52 & 0.07 & 0.13 \\
\hline 4 & 1.44 & 0.17 & 0.12 & 0.56 & 0.08 & 0.14 \\
\hline 10 & 1.64 & 0.19 & 0.12 & 0.64 & 0.09 & 0.14 \\
\hline
\end{tabular}

\section{Appendix C. Ground Motion Selection}

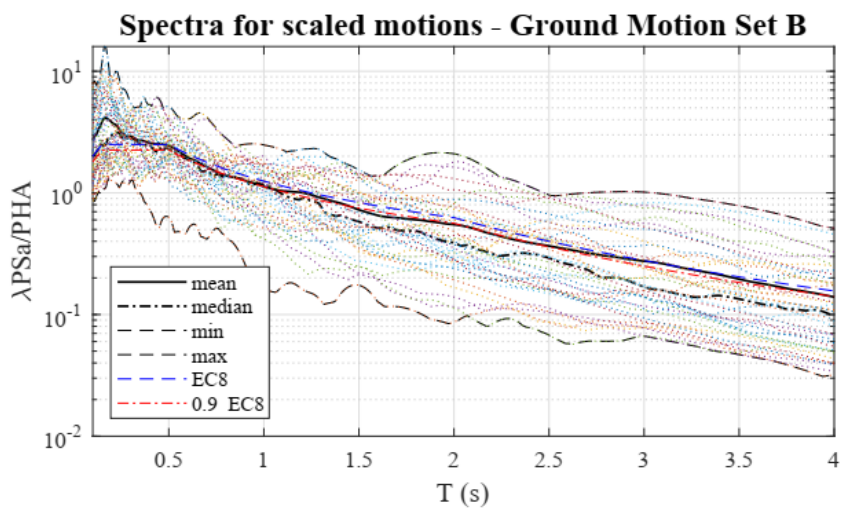

(a)

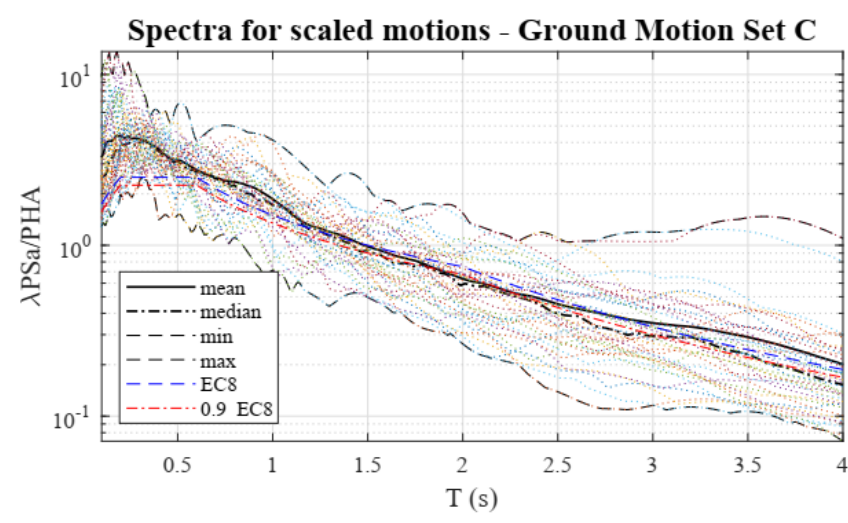

(b)

Figure A2. Pre-scaled and normalized Sa spectra for Ground Motion Selection and comparison with Eurocode 8 5\% elastic response spectrum; (a) GMS B; (b) GMS C. 
Table A2. Selection and pre-scaling of accelerograms for Ground Motion Set B.

\begin{tabular}{|c|c|c|c|c|c|c|c|c|c|}
\hline Name & Station & Date & Fault & Mw & $\begin{array}{l}\text { Epic. } \\
\text { Dist. } \\
(\mathbf{k m})\end{array}$ & $\underset{\text { (g) }}{\text { PHAX }}$ & $\underset{\text { (g) }}{\text { PHAY }}$ & $\underset{\text { (1) }}{\text { Prescale } \lambda_{x}}$ & $\underset{\text { (1) }}{\text { Prescale } \lambda_{y}}$ \\
\hline Friuli (aftershock) & Forgaria-Cornio & 15.09 .1976 & thrust & 6 & 17 & 0.264 & 0.218 & 2.861 & 2.085 \\
\hline Friuli (aftershock) & Forgaria-Cornio & 15.09 .1976 & thrust & 6 & 17 & 0.346 & 0.336 & 2.230 & 1.537 \\
\hline Montenegro & Bar-SkupstinaOpstine & 15.04 .1979 & thrust & 6.9 & 16 & 0.375 & 0.363 & 0.909 & 0.720 \\
\hline Montenegro (aftershock) & Tivat-Aerodrom & 24.05 .1979 & thrust & 6.2 & 21 & 0.166 & 0.133 & 2.481 & 2.198 \\
\hline Montenegro (aftershock) & Petrovac-Hotel Oliva & 15.05.1979 & oblique & 5.8 & 24 & 0.099 & 0.089 & 1.451 & 1.211 \\
\hline Campano Lucano & Calitri & 23.11.1980 & normal & 6.9 & 16 & 0.156 & 0.176 & 0.707 & 0.768 \\
\hline Kalamata & Kalamata-Prefecture & 13.09 .1986 & normal & 5.9 & 10 & 0.215 & 0.297 & 0.819 & 0.982 \\
\hline Kyllini & Zakynthos-OTE Building & 16.10 .1988 & strike slip & 5.9 & 14 & 0.151 & 0.146 & 0.685 & 1.367 \\
\hline Erzincan & Erzincan-Meteorologij & 13.03 .1992 & strike slip & 6.6 & 13 & 0.389 & 0.513 & 0.765 & 1.164 \\
\hline Tithorea & Aigio-OTE Building & 18.11.1992 & normal & 5.9 & 25 & 0.038 & 0.028 & 0.807 & 1.932 \\
\hline Umbria Marche & Gubbio-Piana & 26.09.1997 & normal & 6 & 38 & 0.091 & 0.097 & 0.838 & 0.760 \\
\hline Potenza & Brienza & 05.05 .1990 & strike slip & 5.8 & 28 & 0.096 & 0.080 & 2.208 & 1.534 \\
\hline AnoLlosia & Athens 2 (Chalandri District) & 07.09.1999 & normal & 6 & 20 & 0.110 & 0.161 & 2.121 & 2.411 \\
\hline Griva & Edessa-Prefecture & 21.12 .1990 & normal & 6.1 & 36 & 0.101 & 0.096 & 0.899 & 1.100 \\
\hline South Aegean & Heraklio-Technical University & 23.05.1994 & oblique & 6.1 & 45 & 0.061 & 0.041 & 1.212 & 0.814 \\
\hline Strofades & Zakynthos-OTE Building & 18.11 .1997 & oblique & 6.6 & 38 & 0.131 & 0.116 & 1.152 & 1.542 \\
\hline Kozani & Kastoria-OTE Building & 13.05.1995 & normal & 6.5 & 50 & 0.019 & 0.020 & 1.024 & 1.272 \\
\hline Aigion & Patra-San Dimitrios Church & 15.06 .1995 & normal & 6.5 & 43 & 0.084 & 0.093 & 0.713 & 0.976 \\
\hline Duzce 1 & LDEO Station No C1058 BV & 12.11.1999 & oblique & 7.2 & 11 & 0.111 & 0.073 & 1.257 & 1.044 \\
\hline Firuzabad & Firoozabad & 20.06 .1994 & strike slip & 5.9 & 22 & 0.250 & 0.278 & 3.366 & 3.481 \\
\hline
\end{tabular}

Table A3. Selection and pre-scaling of accelerograms for Ground Motion Set C.

\begin{tabular}{|c|c|c|c|c|c|c|c|c|c|}
\hline Name & Station & Date & Fault & Mw & $\begin{array}{l}\text { Epic. } \\
\text { Dist. } \\
(\mathbf{k m})\end{array}$ & $\underset{\text { (g) }}{\text { PHAX }}$ & $\underset{\text { (g) }}{\text { PHAY }}$ & $\underset{\text { (1) }}{\text { Prescale } \lambda_{x}}$ & $\underset{\text { (1) }}{\text { Prescale } \lambda_{y}}$ \\
\hline Ionian & Lefkada-OTE Building & 04.11 .1973 & thrust & 5.8 & 15 & 0.525 & 0.255 & 1.576 & 1.604 \\
\hline Friuli & Buia & 15.09 .1976 & thrust & 6 & 11 & 0.083 & 0.090 & 1.472 & 1.858 \\
\hline Volvi & Thessaloniki-City Hotel & 20.06 .1978 & normal & 6.2 & 29 & 0.139 & 0.146 & 1.612 & 1.741 \\
\hline Alkion & Korinthos-OTE Building & 24.02 .1981 & normal & 6.6 & 20 & 0.230 & 0.299 & 1.394 & 1.900 \\
\hline Alkion & Korinthos-OTE Building & 25.02 .1981 & normal & 6.3 & 25 & 0.117 & 0.120 & 1.336 & 1.161 \\
\hline Spitak & Gukasian & 07.12 .1988 & thrust & 6.7 & 36 & 0.183 & 0.183 & 1.708 & 1.427 \\
\hline Spitak (aftershock) & Gukasian & 07.12 .1988 & thrust & 5.8 & 36 & 0.147 & 0.103 & 1.896 & 1.987 \\
\hline Umbria Marche & Matelica & 26.09 .1997 & normal & 5.7 & 27 & 0.046 & 0.049 & 2.235 & 2.241 \\
\hline Umbria Marche & Matelica & 26.09 .1997 & normal & 6 & 27 & 0.117 & 0.109 & 2.409 & 2.088 \\
\hline Umbria Marche (aftershock) & Colfiorito & 14.10 .1997 & normal & 5.6 & 13 & 0.092 & 0.068 & 1.705 & 1.450 \\
\hline Sicilia-Orientale & Catania-Piana & 13.12 .1990 & strike slip & 5.6 & 24 & 0.253 & 0.182 & 4.148 & 3.449 \\
\hline Izmit & Yarimca-Petkim & 17.08.1999 & strike slip & 7.6 & 20 & 0.296 & 0.244 & 1.178 & 1.107 \\
\hline Duzce & Bolu-Bayindirlik & 12.11.1999 & oblique & 7.2 & 39 & 0.745 & 0.800 & 1.803 & 1.864 \\
\hline Ano Liosia & Athens-Neo Psihiko & 07.09 .1999 & normal & 6 & 19 & 0.083 & 0.101 & 2.122 & 1.999 \\
\hline Cubuklu & Muradiye-Meteoroloji & 20.04.1998 & oblique & 5.5 & 34 & 0.042 & 0.045 & 1.181 & 0.902 \\
\hline Filippias & Lefkada-Hospital & 16.06 .1990 & thrust & 5.5 & 44 & 0.020 & 0.031 & 1.121 & 1.197 \\
\hline Kyllini & Pyrgos-Agriculture Bank & 16.10 .1988 & strike slip & 5.9 & 49 & 0.025 & 0.030 & 1.357 & 1.847 \\
\hline Duzce 3 (aftershock) & Duzce-Meteoroloji & 23.08 .2000 & strike slip & 5.5 & 41 & 0.023 & 0.017 & 3.166 & 2.522 \\
\hline Bovec & Gemona-Scugelars & 12.04 .1998 & strike slip & 5.6 & 38 & 0.024 & 0.040 & 2.044 & 4.122 \\
\hline Izmit (aftershock) & Yarimca-Petkim & 13.09 .1999 & oblique & 5.8 & 27 & 0.088 & 0.092 & 1.742 & 1.745 \\
\hline
\end{tabular}

(1) Prescale factor. 


\section{Appendix D. Additional Plots}

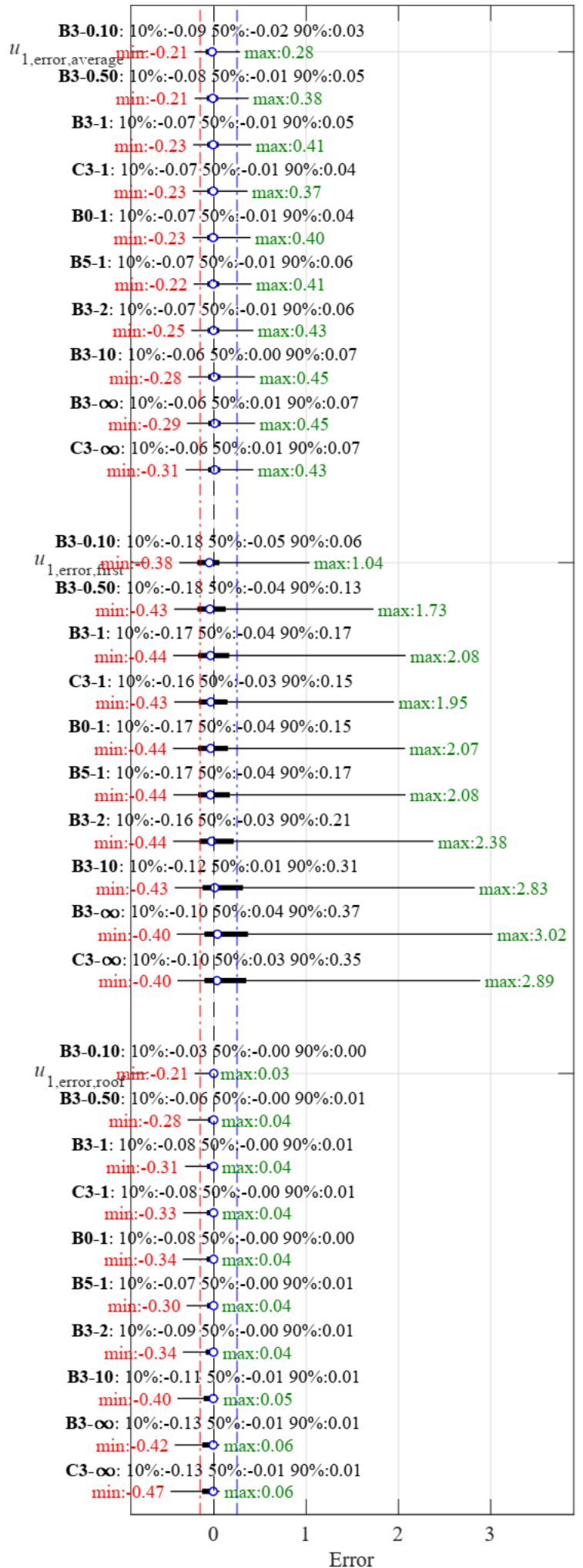

(a)

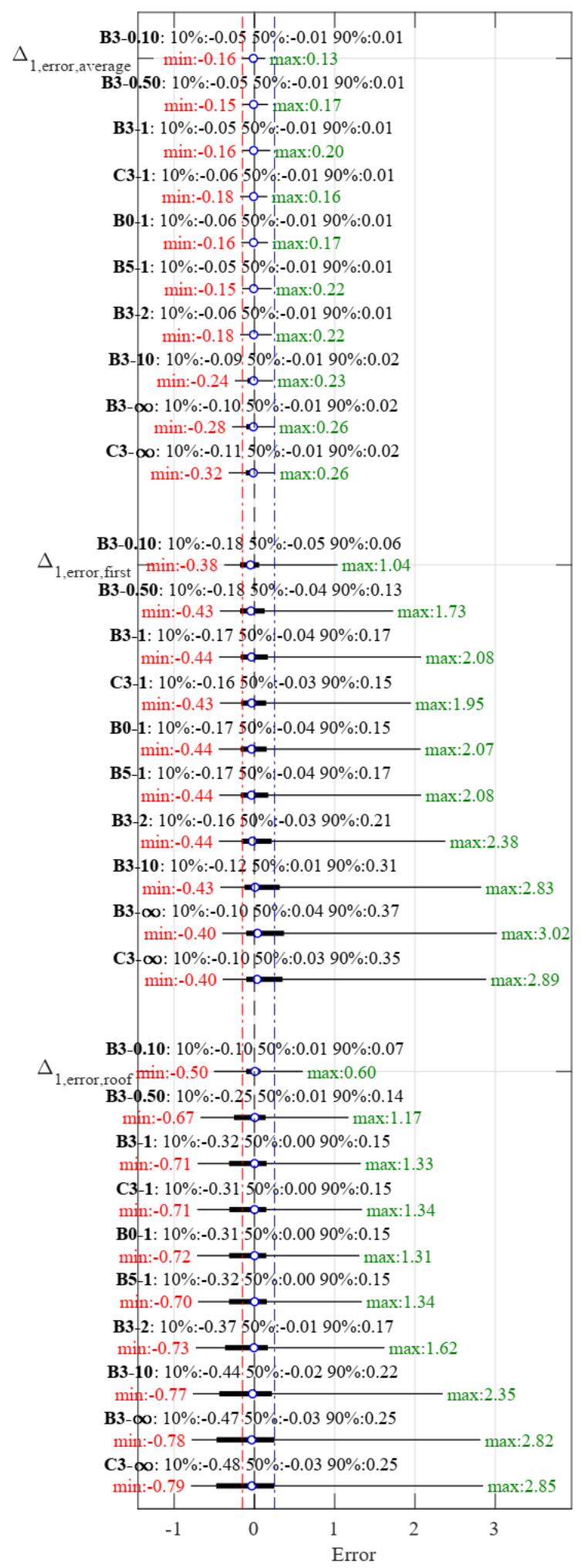

(b)

Figure A3. Bar plot for error type 1, average of stories, first story and roof: (a) relative displacement $u_{\text {error, } 1}$; (b) inter-story drift $\Delta_{\text {error,1 }}$. 


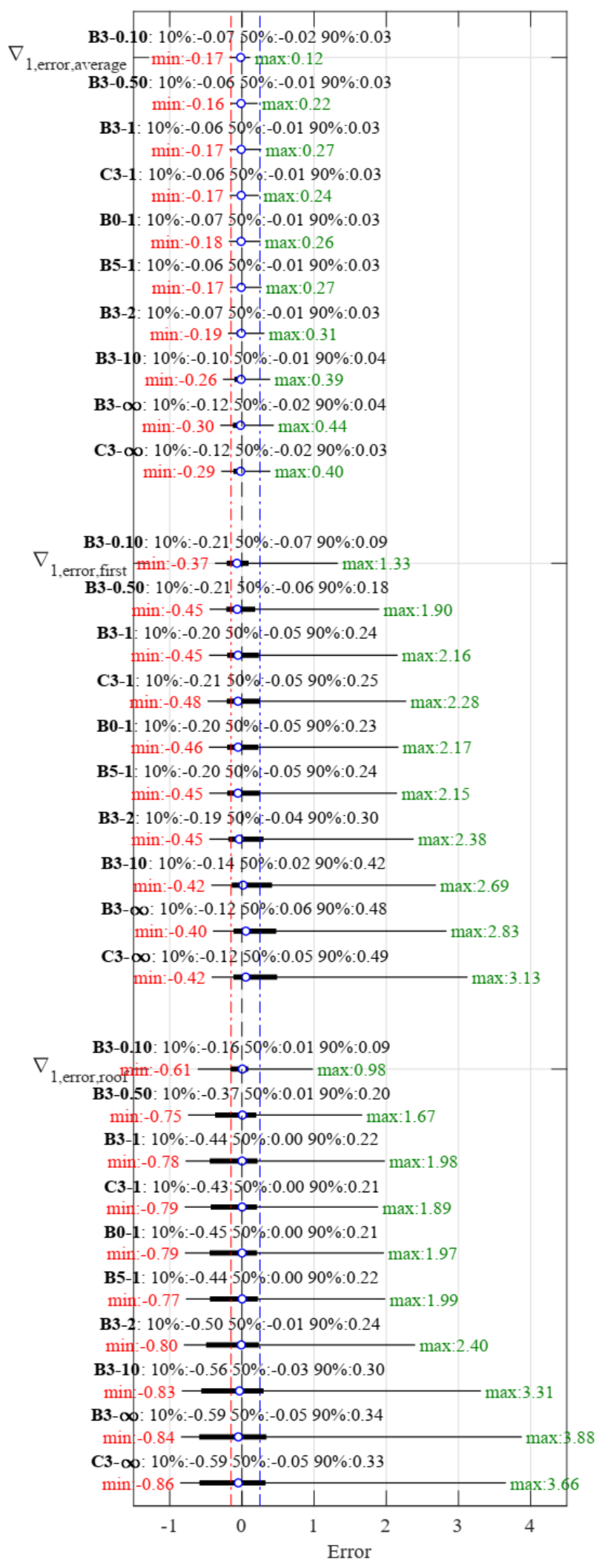

(a)

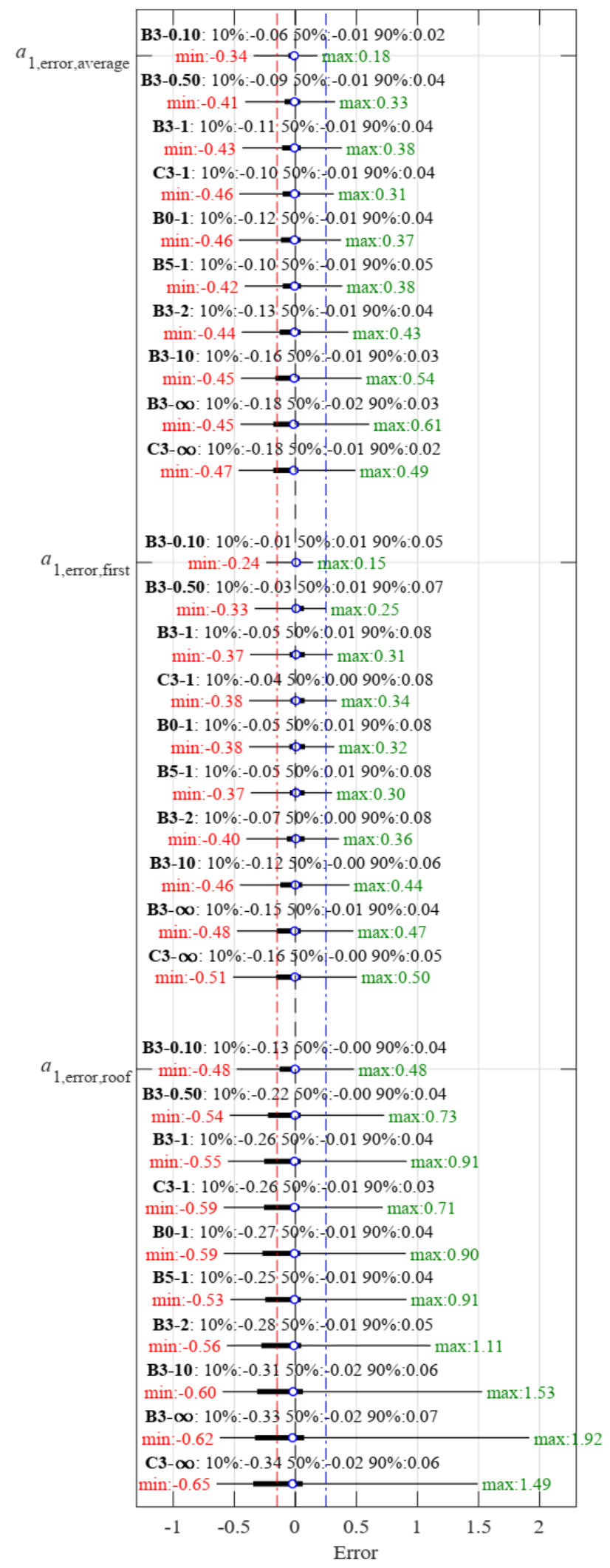

(b)

Figure A4. Bar plot for error type 1, average of stories, first story and roof: (a) inter-story drift velocity $\nabla_{\text {error,1; }}$ (b) absolute acceleration $a_{\text {error, } 1}$. 


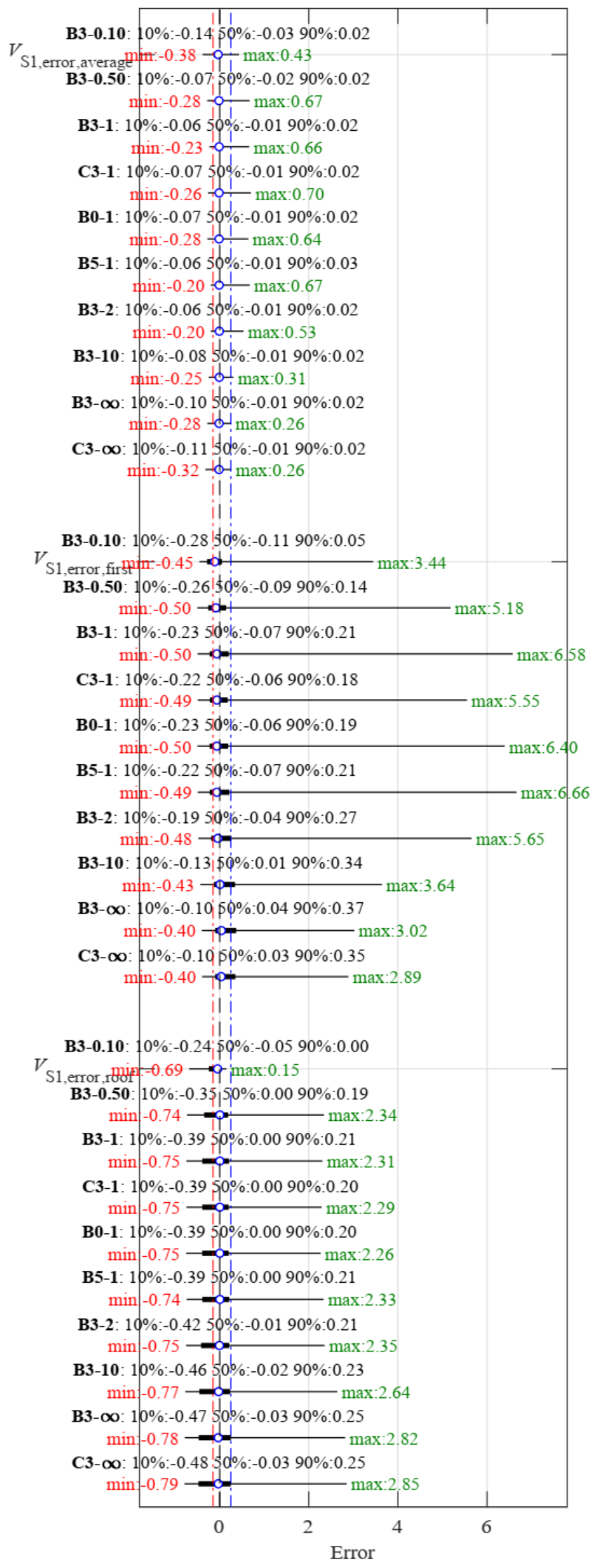

(a)

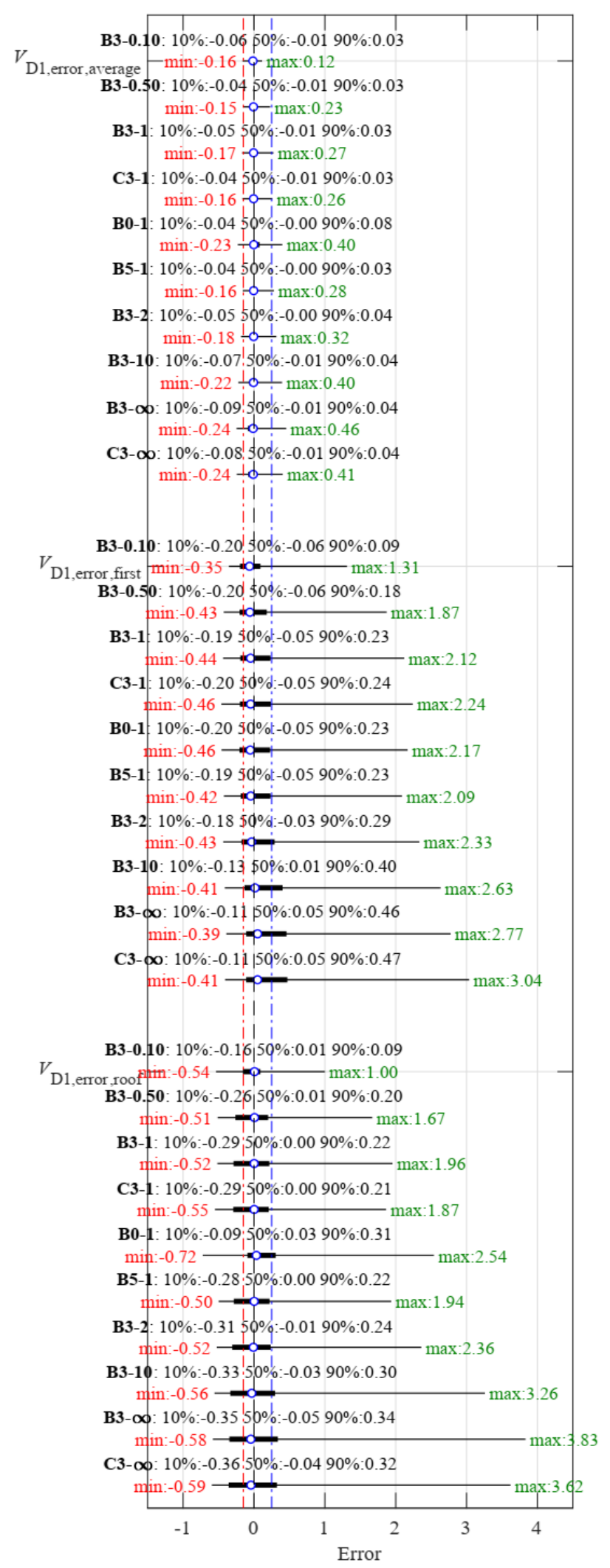

(b)

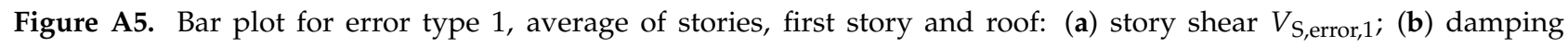
shear $V_{\mathrm{D}, \mathrm{error}, 1}$. 


\section{References}

1. Soong, T.; Dargush, G.F. Passive Energy Dissipation Systems in Structural Engineering, 1st ed.; Wiley: Hoboken, NJ, USA, 1997.

2. Christopoulos, C.; Filiatrault, A. Principles of Passive Supplemental Damping and Seismic Isolation; Iuss Press: Pavia, Italy, 2006.

3. Symans, M.D.; Charney, F.A.; Whittaker, A.S.; Constantinou, M.C.; Kircher, C.A.; Johnson, M.W.; McNamara, R.J. Energy dissipation systems for seismic applications: Current practice and recent developments. J. Struct. Eng. 2008, 134, 3-21. [CrossRef]

4. Constantinou, M.C.; Symans, M.D. Experimental and Analytical Investigation of Seismic Response of Structures with Supplemental Fluid Viscous Dampers; National Center for Earthquake Engineering Research: Buffalo, NY, USA, 1992.

5. Seleemah, A.A.; Constantinou, M.C. Investigation of Seismic Response of Buildings with Linear and Nonlinear Fluid Viscous Dampers; National Center for Earthquake Engineering Research: Buffalo, NY, USA, 1997.

6. Caughey, T.K.; O’Kelly, M.E.J. Classical normal modes in damped linear dynamic systems. J. Appl. Mech. 1965, 32, 583-588. [CrossRef]

7. Federal Emergency Management Agency. FEMA 273: NEHRP Guidelines for the Seismic Rehabilitation of Buildings; FEMA: Washington, DA, USA, 1997.

8. American Society of Civil Engineers (ASCE), A.S.O.C. Minimum Design Loads and Associated Criteria for Buildings and Other Structures; American Society of Civil Engineers (ASCE): Reston, VA, USA, 2017. [CrossRef]

9. Labbé, P. Outlines of the revision of the Eurocode 8, part 1-generic clauses. In Proceedings of the 16th European Conference on Earthquake Engineering, Thessaloniki, Greece, 18-21 June 2018.

10. Foss, K.A. Coordinates which uncouple the equations of motion of damped linear dynamic systems. J. Appl. Mech. 1958, 25, 361-364. [CrossRef]

11. Veletsos, A.S.; Ventura, C.E. Modal analysis of non-classically damped linear systems. Earthq. Eng. Struct. Dyn. 1986, 14, 217-243. [CrossRef]

12. Hanson, R.D.; Soong, T.T. Seismic Design with Supplemental Energy Dissipation Devices; Earthquake Engineering Research Institute: Oakland, CA, USA, 2001.

13. Ramirez, O.M.; Constantinou, M.C.; Kircher, C.A.; Whittaker, A.; Johnson, M.; Gomez, J.D.; Chrysostomou, C.Z. Development and Evaluation of Simplified Procedures of Analysis and Design for Structures with Passive Energy Dissipation Systems; Technical Report No. MCEER-00-0010, Revision 1; Multidisciplinary Center for Earthquake Engineering Research, University of Buffalo: Buffalo, NY, USA, 2001.

14. Clough, R.W.; Penzien, J. Dynamics of Structures, 3rd ed.; Computers and Structures: Berkeley, CA, USA, 2013.

15. Kasai, K.; Fu, Y.; Watanabe, A. Passive Control Systems for Seismic Damage Mitigation. J. Struct. Eng. 1998, 124, 501-512. [CrossRef]

16. Guo, J.W.W.; Christopoulos, C. Performance Spectra Based Method for the Seismic Design of Structures equipped with Passive Supplemental Damping Systems. Earthq. Eng. Struct. Dyn. 2013, 42, 935-952. [CrossRef]

17. Chopra, A.K. Dynamics of Structures: Theory and Applications to Earthquake Engineering, 3rd ed.; Pearson Prentice Hall: Hoboken, NJ, USA, 2007.

18. Caughey, T.K. Classical normal modes in damped linear dynamic systems. J. Appl. Mech. 1960, 27, 269-271. [CrossRef]

19. Goodman, L.E.; Rosenblueth, E.; Newmark, N.M. Aseismic Design of Elastic Structures Founded on Firm Ground; Civil Engineering Studies SRS-026; University of Illinois Engineering Experiment Station, College of Engineering, University of Illinois at UrbanaChampaign: Urbana, IL, USA, 1952.

20. Der Kiureghian, A. On Response of Structures to Stationary Excitation; University of California, Earthquake Engineering Research Center: Berkeley, CA, USA, 1979.

21. Der Kiureghian, A. A Response Spectrum Method for Random Vibrations; University of California, Earthquake Engineering Research Center: Berkeley, CA, USA, 1980.

22. Falsone, G.; Muscolino, G. New real-value modal combination rules for non-classically damped structures. Earthq. Eng. Struct. Dyn. 2004, 33, 1187-1209. [CrossRef]

23. De Domenico, D.; Falsone, G.; Ricciardi, G. Improved response-spectrum analysis of base-isolated buildings: A substructure-based response spectrum method. Eng. Struct. 2018, 162, 198-212. [CrossRef]

24. De Domenico, D.; Ricciardi, G.; Takewaki, I. Design strategies of viscous dampers for seismic protection of building structures: A review. Soil Dyn. Earthq. Eng. 2019, 118, 144-165. [CrossRef]

25. Conde, J. Seismic Response of Structures Equipped with Passive Energy Dissipation Systems Using Simplified Methods Based on Equivalent Effective Properties. Ph.D. Thesis, Universidad Politécnica de Madrid (UPM), Madrid, Spain, 2020. [CrossRef]

26. Liang, Z.; Lee, G.C. Damping of Structures: Part 1-Theory of Complex Damping; National Center for Earthquake Engineering Research, University of Buffalo: Buffalo, NY, USA, 2001.

27. Luo, D.Z. A graphic explanation of undamped and damped mode shapes and its application. J. Sound Vib. 1989, 135, 351-356. [CrossRef]

28. Warburton, G.B.; Soni, S.R. Errors in response calculations for non-classically damped structures. Earthq. Eng. Struct. Dyn. 1977, 5, 365-376. [CrossRef]

29. Prater, G., Jr.; Singh, R. Quantification of the extent of non-proportional viscous damping in discrete vibratory systems. J. Sound Vib. 1986, 104, 109-125. [CrossRef] 
30. Claret, A.M.; Venancio-Filho, F. A modal superposition pseudo-force method for dynamic analysis of structural systems with non-proportional damping. Earthq. Eng. Struct. Dyn. 1991, 20, 303-315. [CrossRef]

31. Tong, M.; Liang, Z.; Lee, G.C. An index of damping non-proportionality for discrete vibrating systems. J. Sound Vib. 1994, 174, 37-55. [CrossRef]

32. Bhaskar, A. Estimates of errors in the frequency response of non-classically damped systems. J. Sound Vib. 1995, 184, 59-72 [CrossRef]

33. Bhaskar, A. Mode shapes during asynchronous motion and non-proportionality indices. J. Sound Vib. 1999, 224, 1-16. [CrossRef]

34. Liu, K.; Kujath, M.R.; Zheng, W. Quantification of non-proportionality of damping in discrete vibratory systems. Comput. Struct. 2000, 77, 557-570. [CrossRef]

35. Liu, K.; Kujath, M.R.; Zheng, W. Evaluation of damping non-proportionality using identified modal information. Mech. Syst. Signal Process. 2001, 15, 227-242. [CrossRef]

36. Prells, U.; Friswell, M.I. A measure of non-proportional damping. Mech. Syst. Signal Process. 2000, 14, 125-137. [CrossRef]

37. Adhikari, S. Optimal complex modes and an index of damping non-proportionality. Mech. Syst. Signal Process. 2004, 18, 1-27. [CrossRef]

38. Nair, S.S.; Singh, R. Examination of the validity of proportional damping approximations with two further numerical indices. J. Sound Vib. 1986, 104, 348-350. [CrossRef]

39. Conde, J.; Bernabeu, A. Influence of Maxwell Stiffness in Damage Control and Analysis of Structures with Added Viscous Dampers. Appl. Sci. 2021, 11, 3089. [CrossRef]

40. Ambraseys, N.; Smit, P.; Douglas, J.; Margaris, B.; Sigbjörnsson, R.; Olafsson, S.; Suhadolc, P.; Costa, G. Internet site for European strong-motion data. Boll. Geofis. Teor. Appl. 2004, 45, 113-129.

41. ESD, European Strong-Motion Database. Available online: http://www.isesd.hi.is/ESD_Local/frameset.htm (accessed on 9 February 2021).

42. European Committee for Standardization. En-1998-1: 2004. Eurocode 8: Design of Structures for Earthquake Resistance. Part 1: General Rules, Seismic Actions and Rules for Buildings; CEN: Brussels, Belgium, 2004.

43. Nigam, N.C.; Jennings, P.C. Calculation of response spectra from strong-motion earthquake records. Bull. Seismol. Soc. Am. 1969, 59, 909-922.

44. Guyader, A.C.; Iwan, W.D. Determining equivalent linear parameters for use in a capacity spectrum method of analysis. J. Struct. Eng. 2006, 132, 59-67. [CrossRef]

45. Nakashima, M.; Ogawa, K.; Inoue, K. Generic frame model for simulation of earthquake responses of steel moment frames. Earthq. Eng. Struct. Dyn. 2002, 31, 671-692. [CrossRef] 